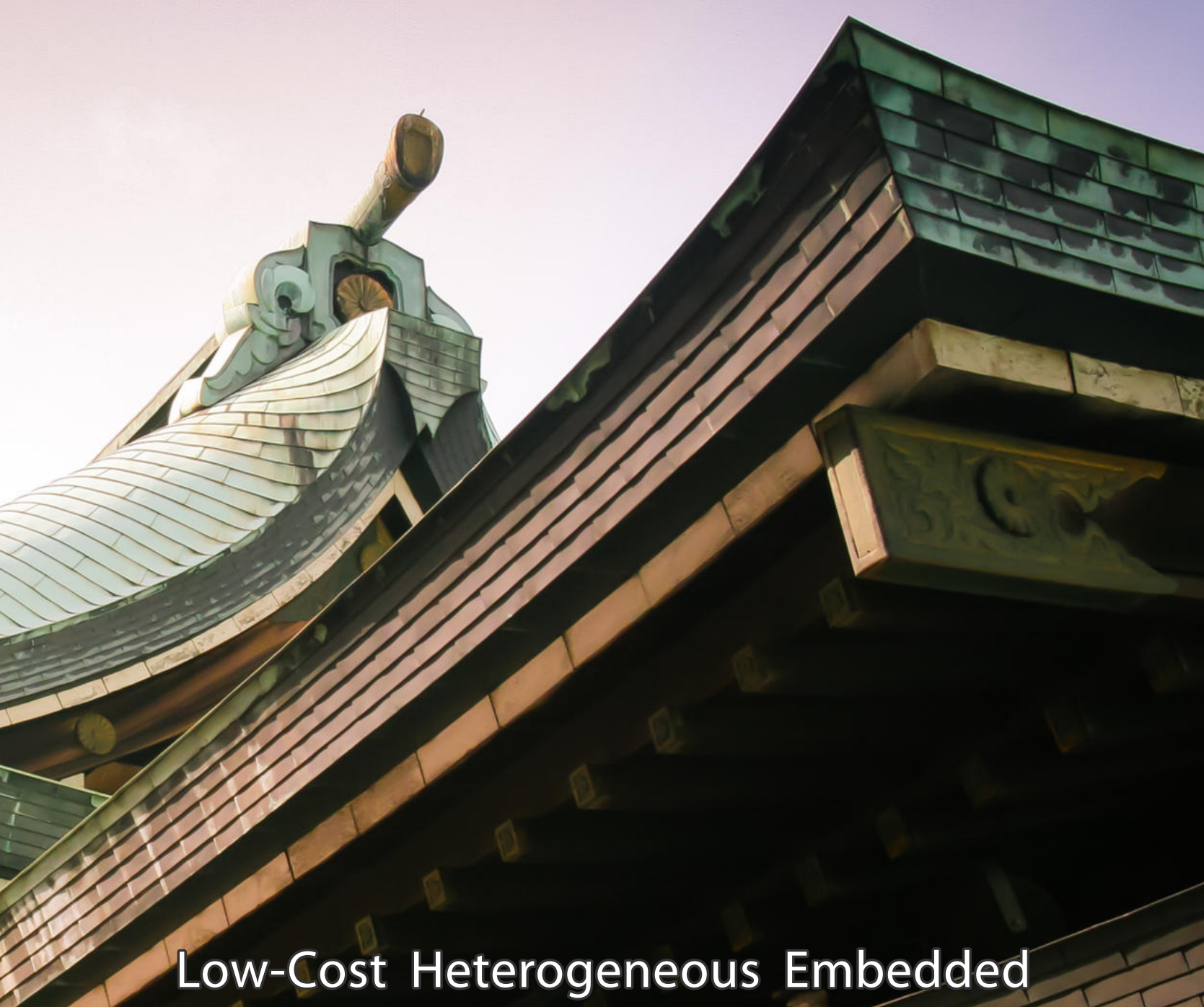
Multiprocessor Architecture for Realetime Stream Processing Applieations 
Members of the graduation committee:

Prof. dr. ir. M. J. G. Bekooij

Prof. dr. ir. G. J. M. Smit

Dr. ir. J. F. Broenink

Prof. dr. ir. K. L. M. Bertels

Prof. dr. ir. D. Stroobandt

Prof. dr.-ing. M. Glaß

Prof. dr. ir. J. van Amerongen
University of Twente (promotor)

University of Twente

University of Twente

Delft University of Technology

Ghent University

Friedrich-Alexander-Universität Erlangen-Nürnberg

University of Twente (chairman and secretary)

\section{UNIVERSITY OF TWENTE.}

Faculty of Electrical Engineering, Mathematics and Computer Science, Computer Architecture for Embedded Systems (CAES) group

CTIT Ph.D. thesis Series No. 15-368

Centre for Telematics and Information Technology

PO Box 217, 7500 AE Enschede, The Netherlands

This research has been conducted within the SenSafety project. This research is supported by the Dutch COMMIT program.

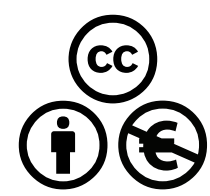

Copyright $\odot 2015$ Berend H.J. Dekens, Enschede, The Netherlands. This work is licensed under the Creative Commons AttributionNonCommercial 3.0 Netherlands License. To view a copy of this license, visit http://creativecommons . org/licenses/by-nc/ $3.0 / \mathrm{nl} /$.

This thesis was typeset using $\mathrm{ETEX}_{\mathrm{E}} \mathrm{X}, \mathrm{TikZ}$, and TeXstudio. This thesis was printed by Gildeprint Drukkerijen, The Netherlands.

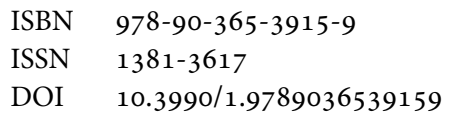




\title{
Low-Cost Heterogeneous Embedded \\ Multiprocessor Architecture for ReAL-Time \\ Stream Processing Applications
}

\author{
DisSERTATION
}

to obtain

the degree of doctor at the University of Twente, on the authority of the rector magnificus, prof. dr. H. Brinksma,

on account of the decision of the graduation committee, to be publicly defended

on Friday $16^{\text {th }}$ October, 2015 at 14:45

by

Berend Hendrik Jan Dekens

born on August 30 ${ }^{\text {th }}, 1983$

in Emmen, The Netherlands 
This dissertation is approved by:

Prof. dr. ir. M. J. G. Bekooij (promotor)

Copyright (C) 2015 Berend H.J. Dekens

ISBN 978-90-365-3915-9 


\section{Abstract}

The time is over for "free" gains in computing power for general purpose processors by simply increasing clock speed and die shrinking. Instead, modern computer architectures are designed to contain multiple processor cores in one chip to improve computing power. Most multi-core architectures can be classified as Symmetric Multi Processor (SMP) or Non-Uniform Memory Architecture (NUMA). Architectures from the SMP class often use homogeneous Processing Elements (PEs) and a shared central memory. As the number of PEs increases, so does the contention for access to the shared memory. This limits the scalability of such a design. The NUMA class of architectures uses multiple PEs where each can have its own local memory. The use of multiple memories will spread the contention for access to memory and improves scalability. At the same time, each memory can have different access latencies from the perspective of the accessing PE. This can influence performance and tends to result in higher programming efforts compared to SMP architectures.

Most homogeneous multi-core architectures employ general purpose processors, which makes them more suited for control-oriented applications than for stream processing applications. Software Defined Radio (SDR) applications are often stream processing applications that are computationally intensive which results in a low throughput on homogeneous multi-core architectures and thus could benefit significantly from the use of stream processing accelerators.

The integration of stream processing accelerators in an architecture is often facilitated by a Network on Chip (NoC). Crossbars or mesh-based NoCs provide guaranteed throughput - as is needed to give the required real-time guarantees for SDR applications - but tend to have unacceptably high hardware costs.

In this thesis a low-cost heterogeneous multi-processor architecture for real-time stream processing applications is proposed together with dataflow models for realtime analysis. This architecture allows compositional temporal dataflow analysis based on independently characterized components.

The proposed architecture is suitable for medium-sized multi-core designs and contains facilities for fine-grained synchronization with a low overhead between stream processing accelerators. The proposed architecture contains aspects from both the SMP and NUMA class as each PE has access to its own local memory but also to a shared central memory where for example instructions can be stored. The proposed architecture contains a low-cost ring-shaped interconnect which provides all-to-all guaranteed throughput communication while being work- 
conserving. Furthermore, cost-effective integration of stream processing accelerators is enabled by combining two low-cost rings and using a small shell in each Network Interface (NI), thereby realizing credit-based hardware flow control for accelerators.

To improve the utilization of stream processing accelerators, we propose a sharing approach to multiplex multiple real-time streams of data over accelerators. Our sharing approach makes use of gateways which work in pairs and multiplex data streams over a number of accelerators.

In this thesis we describe stream processing algorithms as a graph of communicating tasks. Tasks can be implemented in software or hardware. Each software task is required to be side-effect free which allows software tasks to execute using local memory as only local state is modified. Data streams between tasks are transferred using our dual-ring interconnect. Software tasks communicate directly using our distributed software FIFO implementation while communication involving stream processing accelerators is handled by our hardware credit-based flow control.

In order to reason about the worst-case behavior of our architecture, temporal dataflow models are constructed to obtain bounds on throughput and latency. The software FIFO channel between two tasks is described using a Synchronous Data Flow (SDF) model. We will demonstrate that for communication involving stream processing accelerators an SDF model will not suffice and a Cyclo-Static Data Flow $(C S D F)$ model is required to model the dual ring. Sharing of accelerators between multiple data streams is described in a CSDF model. A refinement theory for dataflow models is applied to the CSDF model of a shared stream processing accelerator to obtain an abstraction in the form of an SDF model to simplify further analysis.

Three case studies have been carried out to evaluate the hardware costs and performance of the proposed architecture. For these case studies, several instances of the proposed architecture have been implemented on a Xilinx Virtex-6 FPGA. For the first case study, a multiprocessor instance has been used for a software implementation of a real-time PAL video decoder application based on the PAL broadcasting standard. We demonstrated that a single software task of this decoder can be replaced by a hardware stream processing accelerator with only minor changes to the program description resulting in a $366 \%$ increase in maximum throughput. For the second case study, a multiprocessor instance was used on which a FM stereo audio decoder was implemented. In this decoder, accelerators are shared by multiple data streams, saving over $63 \%$ of hardware costs for accelerators. For the third case study, an instance has been developed for a GMSK radio decoder application. This application enables evaluation of a recently proposed radio standard using a real-time implementation which contains multiple accelerators. An important observation in this case study is that due to the current hardware costs of an entrygateway a reduction in total hardware costs can only be attained when enough and sufficiently large accelerators are shared compared to the case where accelerators are simply duplicated. 
The results from our case studies show that our ring interconnect has a very small hardware cost and performs within the bounds derived by our dataflow analysis models. These bounds can be an over-approximation which becomes smaller as the block sizes increase. We conclude that a considerable reduction of hardware costs can be attained by replacing traditional interconnects by our dual communication ring interconnect. We also conclude that cost-effective shared accelerator integration can improve application performance which demonstrates the merit of our approach. 


\section{SAMENVATTING}

De tijd is voorbij waar we "gratis" meer rekenkracht kregen in centrale processorkernen door simpelweg de kloksnelheid te verhogen of de microchip kleiner te maken. In plaats daar van hebben moderne computer architecturen meerdere processorkernen in één microchip om zo meer rekenkracht te verkrijgen. De meeste multiprocessor architecturen kunnen worden geclassificeerd als "Symmetric Multi Processor (SMP)" of "Non-Uniform Memory Architecture (NUMA)". Architecturen van de SMP-klasse gebruiken vaak homogene processorkernen en een centraal gedeeld geheugen. Bij het toenemen van het aantal processorkernen, neemt de drukte naar het centrale geheugen ook toe. Hierdoor is de schaalbaarheid van deze klasse beperkt. De NUMA klasse ondersteunt het gebruik van meerdere soorten processorkernen waarbij elk verwerkingselement een eigen lokaal geheugen heeft. Door het gebruik van meerdere geheugens wordt de drukte verdeeld en verbetert de schaalbaarheid. Tegelijkertijd betekent dit dat toegang tot een geheugen verschillende wachttijden kan hebben voor elk verwerkingselement. Dit kan de prestaties beïnvloeden en heeft vaak tot gevolg dat het programmeerwerk ingewikkelder is voor de NUMA-klasse dan bij SMP architecturen.

De meeste homogene multiprocessor architecturen gebruiken processoren voor algemeen gebruik. Hierdoor zijn ze beter geschikt voor controle-georiënteerde applicaties dan voor datastroom verwerking. Software Defined Radio (SDR) applicaties zijn radio ontvangers beschreven in software welke vaak rekenintensief zijn wat in een lage doorvoersnelheden resulteert op homogene multiprocessor architecturen. Dit soort applicaties kan enorme verbeteringen in prestatie krijgen door het gebruik van datastroom verwerkende versnellers.

De integratie van datastroom verwerkende versnellers in een architectuur wordt vaak gefaciliteerd door het Netwerk-op-Chip (NoC). Zogenaamde "crossbars" of raster-topologieën geven garanties over de doorvoer - een eigenschap die nodig is om real-time garanties te kunnen geven voor SDR applicaties - maar hebben vaak onacceptabel hoge hardware kosten.

In dit proefschrift wordt een heterogeen multiprocessor architectuur met lage hardware kosten voor real-time datastroom verwerkende applicaties gepresenteerd tezamen met dataflow modellen voor real-time analyse. Deze architectuur staat toe om compositionele temporale dataflow analyse te gebruiken gebaseerd op onafhankelijk gekarakteriseerde componenten.

De voorgestelde architectuur is geschikt voor middelgrote multiprocessor ontwerpen en bevat ondersteuning voor fijnmazige synchronisatie met een lage overhead 
tussen datastroom verwerkende versnellers. De voorgestelde architectuur bevat aspecten van zowel de SMP als NUMA klasse, aangezien elke rekenkern een eigen lokaal geheugen heeft, maar ook een centraal gedeeld geheugen waar bijvoorbeeld instructies kunnen worden opgeslagen. De voorgestelde architectuur bevat een goedkope ring-gebaseerde verbindingsmodule welke ondersteuning voor allenaar-alle gegarandeerde doorvoer communicatie heeft en terwijl het werk behoudend is. Tevens is kost-effectieve integratie van datastroom verwerkende versnellers bewerkstelligd door twee goedkope ring netwerken te combineren tezamen met een kleine schil waarmee een krediet-gebaseerde stroomsturing gemaakt is.

Om datastroom verwerkende versnellers beter te benutten stellen we een aanpak voor het delen van versnellers tussen meerdere real-time datastromen. Onze aanpak maakt gebruik van zogenaamde "gateways" welke in paren samenwerken om datastromen over een aantal versnellers te multiplexen.

In dit proefschrift beschrijven we datastroomverwerkende algoritmes als een graaf bestaande uit communicerende taken. Taken kunnen geïmplementeerd worden in software of hardware. Elke software taak moet vrij zijn van bijwerkingen waardoor software taken uitgevoerd kunnen worden in een lokaal geheugen aangezien alleen lokale toestand wordt aangepast. Datastromen tussen taken worden over onze dubbele ring gestuurd. Softwaretaken communiceren met elkaar door middel van onze gedistribueerde software FIFO implementatie terwijl communicatie met datastroom verwerkende versnellers wordt afgehandeld met onze hardware krediet-gebaseerde stroomsturing.

Om te kunnen redeneren over het slechts mogelijke temporele gedrag van onze architectuur, worden temporele dataflow modellen gebruikt om grenzen aan doorvoer en wachttijden te bepalen. Een software FIFO-kanaal tussen twee taken wordt beschreven door middel van een Synchronous Data Flow (SDF)-model. We zullen aantonen dat voor communicatie met datastroom verwerkende versnellers een SDF-model niet volstaat en een Cyclo-Static Data Flow (CSDF)-model nodig is om het dubbel ring netwerk te beschrijven. Het delen van versnellers tussen meerdere datastromen is beschreven in een CSDF-model. Een theorie voor verfijning voor dataflow modellen is toegepast op het CSDF-model van een gedeelde versneller om een abstractie te maken in de vorm van een SDF-model welke verdere analyse versimpelt.

Drie studies zijn uitgevoerd om de hardware kosten en prestaties van de voorgestelde architectuur te evalueren. Er zijn meerdere instanties van de voorgestelde architectuur gemaakt voor deze studies op een Xilinx Virtex-6 FPGA. Voor de eerste studie wordt een multiprocessor instantie gebruikt in een software implementatie van een real-time $P A L$-video decoder applicatie gebaseerd op de PAL-uitzendingenstandaard. We laten zien dat een enkele software taak van deze decoder kan worden vervangen door een hardware datastroom verwerkende versneller met kleine aanpassingen aan het programma waarmee een $366 \%$ verbetering in doorvoersnelheid wordt bewerkstelligd. In de tweede studie wordt een multiprocessor instantie gebruikt voor de implementatie van een FM stereo audio decoder. In deze decoder 
worden versnellers gedeeld door meerdere datastromen waarbij meer dan $63 \%$ van de hardware kosten van versnellers wordt bespaard. In de derde studie werd een instantie ontwikkeld voor een GMSK radio decoder applicatie. Met behulp van deze applicatie kan een voorgestelde, nieuwe radio-standaard worden geëvalueerd door middel van een real-time implementatie welke meerdere versnellers bevat. Een belangrijke observatie uit deze case study is dat door de huidige kosten van de ingangs-gateway een reductie in de totale hardware kosten alleen behaald kan worden wanneer voldoende en afdoende groote versnellers gedeeld worden ten opzichte van de situatie dat versnellers simpelweg gedupliceerd worden.

De resultaten van onze studies laten zien dat ons ring netwerk erg goedkoop is en presteert binnen de grenzen die afgeleid zijn uit de analyse van onze dataflow modellen. Deze grenzen kunnen een overschatting zijn welke kleiner worden als blokgroottes toenemen. We concluderen dat een behoorlijke reductie in de hardware kosten behaald kan worden door een traditionele verbindingsmodule te vervangen door onze dubbele ring. Tevens concluderen we dat kost-effectieve integratie voor datastroom verwerkende versnellers de prestaties van applicaties kan verbeteren wat aantoont dat onze aanpak waardevol is. 


\section{DANKWOORD}

$\mathrm{Na}$ iets meer dan vier jaar werk kan ik eindelijk mijn dankwoord schrijven. En waar het dankwoord vaak wordt geschreven terwijl men nog werkt op de universiteit, schrijf ik dit vanuit een nieuw land. Maar wanneer ik terug kijk naar mijn tijd op de universiteit kan ik zeggen dat ik het naar mijn zin heb gehad.

Vaak begint een dankwoord met de historie hoe iemand op het idee is gekomen om te promoveren, dus bij deze. Tijdens mijn afstuderen zat ik bij Marcel en Kenneth op de kamer en kreeg zo wat mee van wat een promovendus zoal doet, behalve natuurlijk koffie drinken. Mijn ambities om te promoveren kreeg ik tijdens het doen van mijn afstudeeropdracht bij Marco Bekooij. Ik kreeg de opdracht om een prototype van een radio-ontvanger met behulp van een experimenteel stuk software op een bijna compleet multi-core systeem te plaatsen, wat zeer waarschijnlijk mogelijk zou moeten zijn. Mijn begeleider tijdens mijn afstuderen, Jochem, had dat multi-core systeem ontworpen gedurende zijn promotie en de complexiteit en mogelijkheden van een dergelijk systeem vond ik zeer interessant. Het duurde dan ook niet lang voordat ik dingen met het systeem deed waar het niet voor ontworpen was, maar wat prima werkte. Ik realiseerde me dat ik als promovendus de mogelijkheid zou krijgen om dieper op de materie in te gaan en toen de kans zich voordeed heb ik deze dan ook aangegrepen.

Ik heb met veel plezier gewerkt aan hetgeen wat in dit boekje beschreven is. Voor elk idee wat we uitgewerkt hebben, hebben we minstens net zo veel nieuwe mogelijke richtingen voor verdere verkenning ontdekt.

De inhoud van dit werk is mogelijk gemaakt door de mensen die direct of indirect hebben bijgedragen. Ten eerste wil ik Jochem bedanken voor de basis van het systeem. Tijdens mijn afstuderen en promotie heb ik meegeholpen met het opsporen van de laatste imperfecties waardoor het systeem nu vast en zeker vrij van fouten is.

De studenten die hebben bijgedragen aan delen van de realisatie: Gerald, Guus, Gerben en Joris bedankt. Ik heb met jullie menige discussie gehad die vaak voor zowel jullie als mijzelf tot nieuwe inzichten hebben geleid.

Degenen die leuke demo's hebben gebouwd met dit systeem: Oscar, Daniel en Harm bedankt. Het is mooi om te zien dat een complex systeem met de juiste abstracties gebruikt kan worden in allerlei applicaties.

Ik wil mijn begeleider Marco Bekooij bedanken voor de vele jaren waarin ik met veel plezier met hem heb samengewerkt, de vele discussies die tot nieuwe inzichten 
hebben geleid en de vele revisies die we gemaakt hebben om de tekst scherp op papier te krijgen.

Graag wil ik mijn collega's van de vakgroep bedanken voor de leuke jaren en de vele koffiepauzes vol zin en onzin, die ik aan het einde van mijn promotie eerlijk gezegd wat te vaak heb gemist.

Ook wil ik graag mijn oude kamergenoten Philip en Robert bedanken voor de leuke gesprekken en discussies die we hebben gehad.

Ik wil Gerard Smit bedanken voor de mogelijkheid om een promotie binnen de vakgroep te mogen doen.

Tevens wil ik de secretaresses bedanken voor hun ondersteuning in allerlei dingen: Marlous, Nicole en Thelma bedankt.

Graag wil ik mijn vrienden bedanken voor de gezellige avonden die we regelmatig tijdens mijn promotie hebben gehad. Ik hoop dat dit boekje meer duidelijkheid kan verschaffen over wat ik nou eigenlijk heb gedaan.

Ik wil mijn ouders bedanken voor de vrijheid en ondersteuning die ik altijd van hun heb gekregen, ongeacht of ik nou wel of niet in begrijpelijke taal kon uitleggen wat ik deed, en mijn zusjes Margriet en Jannemarie die altijd mijn (veel te) technische verhalen aan mochten horen.

Zuletzt möchte ich Anja danken für alles. Nicht nur für das Lesen meines "Meisterwerks" sondern für so viel mehr.

Berend Dekens

Trondheim, Noorwegen

September 2015 


\section{Contents}

1 INTRODUCTION 1

1.1 Background . . . . . . . . . . . . . . . . . . . . . . . . 1

1.2 Programming Multi-core ... . . . . . . . . . . . . 2

1.2.1 Real-time Properties of Architectures .......... 4

1.2.2 Classification of Architectures .............. 7

1.3 Problem Statement . . . . . . . . . . . . . . . . . . 9

1.4 Approach and Contributions . . . . . . . . . . . . . . 10

1.4.1 Contributions . . . . . . . . . . . . . . . 12

1.5 Structure . . . . . . . . . . . . . . . . . . . . 13

2 RELATED WORK 15

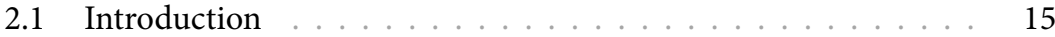

2.2 Background . . . . . . . . . . . . . . . . . . 16

2.3 Modern Stream Processing Architectures . . . . . . . . . 17

2.4 Interconnects . . . . . . . . . . . . . . . . . . . . 20

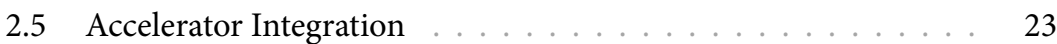

2.5 .1 Co-processors . . . . . . . . . . . . . . . 24

2.5.2 Point-to-point Streaming _. . . . . . . . . . . 24

$2.5 .3 \quad$ Wrappers ......................... 25

2.6 Accelerator Sharing . . . . . . . . . . . . . . . . . 26

2.7 Summary . . . . . . . . . . . . . . . . . . . . . . 27

3 Real-time Stream Processing Architecture 31

3.1 Introduction . . . . . . . . . . . . . . 31

3.2 Architecture Overview . . . . . . . . . . . . . . . . . . 32

3.2.1 Tile-based Architecture . . . . . . . . . . . . . . 33

3.2 .2 Communication .................. 34

3.3 Processor Tile . . . . . . . . . . . . . . . . . . . 35

3.4 Accelerator Tile . . . . . . . . . . . . . . . . . . 36 
3.5 Gateway Tiles . . . . . . . . . . . . . . . . . 37

3.6 Dual-ring Interconnect . . . . . . . . . . . . . . . . 38

3.6.1 Single Ring Design . . . . . . . . . . . . . . . . . 39

xvi

3.6.2 Software Flow Control . . . . . . . . . . . . . . . 41

3.6.3 Hardware Flow Control . . . . . . . . . . . . . . . 42

3.7 Secondary Interconnect for Shared Slaves . . . . . . . . . . . . 43

3.7.1 Shared Memory . . . . . . . . . . . . . . . . . . 44

3.7.2 Other Peripherals . . . . . . . . . . . . . 44

3.8 Programming . . . . . . . . . . . . . . . . . . 45

3.9 Summary . . . . . . . . . . . . . . . . . . . . . . . . . . 46

4 LOW-COST Ring INTERCONNECT 49

4.1 Introduction . . . . . . . . . . . . . . . . . . . . . . . . . . . 49

4.2 Ring Design . . . . . . . . . . . . . . . . . . . . . 50

4.3 Ring Slotting . . . . . . . . . . . . . . . . . . . . 51

4.4 Hardware Design . . . . . . . . . . . . . . . . . . . 51

4.5 Temporal SDF Model . . . . . . . . . . . . . . . . . 53

4.5.1 Work-conserving . . . . . . . . . . . 57

4.5.2 External Memory . . . . . . . . . . . . . . 57

4.6 Hardware Costs . . . . . . . . . . . . . . . . . . . . . 58

4.6.1 Synthesis Results and Power Estimates for ASICs . . . . . . 58

4.7 Design Improvements . . . . . . . . . . . . . . . . 59

4.7.1 Reorder Slots to Improve Average Latency . . . . . . . . 59

4.7.2 Reorder Slots to Improve Power Efficiency . . . . . . . . . 60

$4.7 .3 \quad$ Clock Gating . . . . . . . . . . . . . . . . 60

4.7.4 Slot Masking . . . . . . . . . . . . . . . . . . 60

$4.7 .5 \quad$ Slot Reallocation . . . . . . . . . . . . . . 61

4.7 .6 Mapping . . . . . . . . . . . . . . . . 61

4.8 Conclusion . . . . . . . . . . . . . . . . . . . 61

5 ACCELERATOR INTEgRATION 63

5.1 Introduction . . . . . . . . . . . . . . . . . . 63

5.2 Single-ring Interconnect . . . . . . . . . . . . . . . . . 64

5.2.1 Credit Ring . . . . . . . . . . . . . . . . . 65

$5.2 .2 \quad$ Ring Shell . . . . . . . . . . . . . . . . . 66

5.3 Dataflow Model . . . . . . . . . . . . . . . . . . . . . 67

5.3.1 Channel Description . . . . . . . . . . . . . . 69

5.3.2 Guaranteed Throughput . . . . . . . . . . . . . 71

5.4 Hardware Costs . . . . . . . . . . . . . . . . . . . . . . . 73 
6 Sharing OF ACCELERATORS $\quad 77$

xvii

6.1 Introduction . . . . . . . . . . . . . . . . 77

6.2 Basic Idea . . .

6.3 Dataflow Model . . . . . . . . . . . . . . . . . . . 81

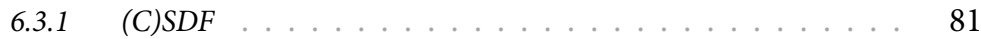

$6.3 .2 \quad$ CSDF Model . . . . . . . . . . . . . . . . 82

6.3.3 Single SDF Actor model . . . . . . . . . . . . 83

6.3.4 Minimum Throughput Verification ............ 84

6.3 .5 Non-monotone Behavior . . . . . . . . . . . 84

6.3.6 Computing Block Sizes . . . . . . . . . . . . . . . . . . 84

6.3 .7 Check for Space . . . . . . . . . . . . . . . . 86

6.4 Evaluation . . . . . . . . . . . . . . . . . . . . . 86

6.5 Future Improvements . . . . . . . . . . . . . . . . . . 88

6.5.1 Gateway Cost Reduction . . . . . . . . . . . . . . 88

6.5.2 Hierarchical Rings . . . . . . . . . . . . . . . . . 88

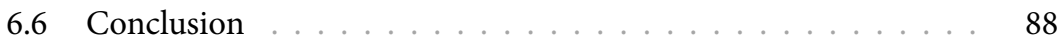

7 Case Study

7.1 PAL Television Decoder . . . . . . . . . . . . . . . . . 94

7.1.1 PAL Video Decoder . . . . . . . . . . . . . . . . . . . 95

7.1.2 Demodulating Video . . . . . . . . . . . . . . . 97

7.1.3 Accelerated Video Decoding . . . . . . . . . . . . . . . 100

7.1.4 Accelerated Stereo Audio Decoding . . . . . . . . . . . . 102

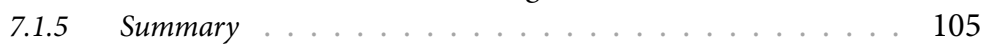

7.2 GMSK Decoder . . . . . . . . . . . . . . . . . . 105

7.2.1 Minimum Shift Keying . . . . . . . . . . . . . . 106

7.2.2 Gaussian Minimum Shift Keying . . . . . . . . . . . 107

7.2 .3 Correlator . . . . . . . . . . . . . . . . . . 108

$7.2 .4 \quad$ Matched Filter . . . . . . . . . . . . . . . . . 109

7.2 .5 Channel Equalizer. . . . . . . . . . . . . . . 110

7.2.6 Forward Error Correction Decoder . . . . . . . . . . 110

7.2 .7 Audio Output . . . . . . . . . . . . . . . . 111

$7.2 .8 \quad$ Results . . . . . . . . . . . . . . . . . 111

7.2 .9 Summary . . . . . . . . . . . . . . . . . 116

7.3 Conclusions . . . . . . . . . . . . . . . . 117 
8 Conclusion 121

8.1 Recapitulation . . . . . . . . . . . . . 123

xviii

8.2 Contributions . . . . . . . . . . . . . . . . 125

8.3 Future Work . . . . . . . . . . . . . . . . . . 128

$\begin{array}{ll}\text { ACronyms } & 133\end{array}$

8
3
4
营
0

LIST OF SyMboLS 135

$\begin{array}{ll}\text { BIBLIOGRAPHY } & 141\end{array}$

$\begin{array}{ll}\text { List of Publications } & 149\end{array}$

$\begin{array}{ll}\text { INDEX } & 151\end{array}$ 


\title{
INTRODUCTION
}

\begin{abstract}
Homogeneous multi-core architectures are usually not used for real-time stream processing applications. These architectures are not very efficient for computationally intensive applications as generic purpose processors are inherently unsuitable for such tasks. Heterogeneous architectures which are designed for stream processing applications are a better solution but tend to require interconnects with support for guaranteed throughput, for example based on crossbars or using mesh-based topologies, which can have high hardware costs. In this thesis we introduce a dual communication ring interconnect for shared accelerator integration which has a much lower hardware cost than the previously mentioned interconnects. This dual-ring interconnect and the sharing support are modeled using a temporal dataflow model which enables the temporal analysis of those components for use with real-time stream processing applications.
\end{abstract}

\subsection{BACKGROUND}

In the early years of computer development, each generation of processors became more complex and faster. This trend has been visible for over $35^{1}$ years, where the speed of processors was increased every year.

While the complexity of the processors and their features increased, so did their computational power. However, often the biggest boost in computational power came simply from increasing the clock speed: executing operations twice as fast doubled the processing power. In the quest for faster processors, this was often considered a "free" performance increase as it only required the hardware to run faster. However, after three decades this effect became one of diminishing returns as circuits become smaller and smaller while it is increasingly more difficult to provide power to transistors and at the same time extract the heat of those same transistors. This effect is known as the "Power Wall" and currently is one of the biggest

\footnotetext{
${ }^{1}$ Which began with the first monolithic CPU from Intel in 1971, the 4004
} 
limiting factors for high frequency IC design. To illustrate the severity of the heating issue, it has been said that the thermal dissipation of modern processors [62] $\left(30 \mathrm{~W} / \mathrm{cm}^{2}\right)$ is comparable to the heat transfer of a nuclear reactor ${ }^{2}\left(100 \mathrm{~W} / \mathrm{cm}^{2}\right)$.

Instead of making single CPUs faster, more processing power can be obtained by simply using multiple CPUs in the same computer chip. In 2005 the first multi-core CPU was produced by Intel and AMD, marking the availability of such technologies for general purpose computers to the public. While multi-core chips have increased available processing power in theory, harvesting this power turned out to be difficult as most programming languages using the imperative programming paradigm like Basic, $\mathrm{C}$ and Java. This allows the programmer to specify algorithms in a sequential manner.

\subsection{Programming Multi-CORE}

The previously mentioned programming languages fall within the imperative programming paradigm ${ }^{3}$ in which a program is expressed as a sequential list of statements. This programming paradigm is very well suited for a single CPU because by definition it describes how the state of a state-machine should be updated during program execution. As a result, these sequential descriptions do not fit well when used with multi-core CPUs. Compilers have been improved over the years to detect potential instruction or data level parallelism which can be exploited using special instructions or by reordering statements. While this has improved processing performance for a single core, it is sometimes impossible to detect data dependencies between statements, making parallel execution often very difficult [34]. Executing larger segments of a program in parallel - for example loop iterations, resulting in a large amount of task-level parallelism - requires that the data dependencies between such a segment of code and the rest of the program is explicit and is therefore often not possible using the program description alone. In order to allow algorithms to be properly converted into programs which work on multiple cores, a different approach is needed.

One solution is to try and amend the problem in existing languages by extending them with constructs to describe potential parallelism. OpenMP [57] is probably a well known extension for the $\mathrm{C} / \mathrm{C}++$ and Fortran languages which allows a programmer to augment source code with hints to the compiler to explicitly determine which code segments have no external data dependencies (aside from those explicitly marked) and as such can be executed in parallel. The main issue with this approach is that incorrect use of these hints results in data sharing violations that sometimes will not be detected by the compiler and as such results in programs which compile correctly but do not function as intended.

\footnotetext{
${ }^{2}$ See $[52]$, page 5

${ }^{3}$ The latest versions of $\mathrm{C}++$ and Java contain elements from the functional programming paradigm but were originally purely imperative.
} 
Another solution is to restrict the available syntax or take a subset of a language, which is for example done in Single Assignment C [69]. By allowing variables to be explicitly (e.g. no pointers) assigned a value once, dependencies between statements can be resolved at compile time. This allows the compiler to reorder statements as long as data dependencies are not violated and potentially execute statements in parallel. The actual extraction of potential parallelism and the mapping onto multiple threads is left to the compiler.

Programming languages like $\mathrm{SaC}$ are no longer imperative, instead they fall in the realm of functional programming. In the imperative programming paradigm, a programmer writes code which makes up a step-by-step guide for the computer to achieve a goal. Functional languages like Haskell on the other hand, consist of the composition of a set of functions. In essence, the imperative approach gives the computer a step-by-step guide to reach a goal whereas the functional approach leaves the ordering of steps up to the compiler. The main advantage of function composition is that data dependencies between statements are clearly visible in the language. As such, parallel execution is possible but now clustering of statements has to be applied to prevent executing trivial instruction in parallel which would result in programs with unacceptably high synchronization overhead.

An important issue arises from the support for "lazy" evaluation: programs in functional languages can implicitly require an infinite amount of memory for their execution. This means that the execution schedule of such programs can only be determined at run-time. It also means that the amount of memory required can potentially be unbounded, a property which is highly undesirable for safety critical embedded systems.

Coordination languages are orthogonal compared to other programming models as they treat process coordination as a separate activity from computation. This means that computation is allowed to be expressed in other programming languages. In a coordination language primitives can operate on an ordered sequence of data objects, similar to stream processing.

Definition 1.1 We define stream processing as a programming paradigm where a number of computation units process a potentially infinite stream of data in sequence. This paradigm matches closely with most algorithms from the Software Defined Radio (SDR) domain as well as other domains such as audio and video processing.

A program expressed in a coordination language describes the process coordination as communication between computation units. Computational units are presumed to be side effect free: aside from explicit data passing as described using the coordination language, one unit can not influence the functional behavior of another unit implicitly. This program description can be transformed into a directed task graph which is mapped onto multiple processors.

For the programming of the proposed multi-processor architecture the stream processing applications could be described using the coordination language OIL [23]. 
Unlike functional languages like Haskell, a program description written in OIL defines a valid execution schedule for all tasks which can be extracted at compile time. An important implication is that the maximum amount of required memory can be determined and as such compiled programs execute in bounded memory. Additionally, the OIL compiler (Omphale) can guarantee that the compiled program will be deadlock-free. Such properties are essential for programs used in safety critical embedded systems.

While the OIL language only describes a program consisting of communicating tasks or kernels, this description lends itself well for data-oriented applications with a low amount of control, like stream processing applications. Because OIL only defines that tasks are side effect free it allows embedding of tasks written in various languages. A practical advantage of this approach is the enabling of the use of legacy code.

The OIL language has many features similar to functional programming languages like Haskell. In both languages, a variable does not denote a memory address but a data dependency between statements. As a consequence there is no memory consistency model defined for these languages. Data dependencies can always be resolved, just like in a functional language, at least up to the array being addressed. However, the exact element in the array being addressed can not always be determined at compile time.

Differences between OIL and a functional language also exist. In Haskell, when using the IO Monad, the order in which I/O variables are written is defined in the program description. This means that pipelining is not possible and as such it is not suitable for stream processing applications.

The translation from the program description to a task graph which is suitable for parallel execution and a corresponding dataflow graph is defined for OIL. Such a transformation does not yet exist for a description in a functional language like Haskell.

We can compare the properties of these types of programming languages as is shown in Table 1.1. The "acceptance level" of a language type is based on the popularity of the type. When we consider a coordination language like OIL, we get the advantages of a functional language to describe the top level of an application with the familiarity of an imperative language for the tasks of a program which could aid in the acceptance of a new language.

\subsubsection{Real-time Properties of Architectures}

In order for an architecture to be suitable for real-time applications, all components used should have a predictable worst-case bound. This means that for each component a temporal model should be available which allows efficient analysis to derive sufficiently tight bounds on the worst-case temporal behavior.

\footnotetext{
${ }^{4}$ Based on the assumption that semantics expressing a step-by-step recipe for an algorithm similar to an imperative language results in easy acceptance
} 


\begin{tabular}{rccc}
\hline & $\begin{array}{c}\text { Suitable for } \\
\text { multi-core }\end{array}$ & $\begin{array}{c}\text { Acceptance } \\
\text { level }\end{array}$ & $\begin{array}{c}\text { Match stream } \\
\text { processing }\end{array}$ \\
\hline Imperative Languages & - & + & - \\
Functional Languages & + & - & - \\
OIL Coordination Language & + & $+^{4}$ & + \\
\hline
\end{tabular}

TABLE 1.1 - Comparison of types of programming languages

The concept of real-time means that data entering the system has to be processed before a certain deadline expires, as is illustrated in Figure 1.1(a). When real-time behavior is not required, a system is using best effort, as is shown in Figure 1.1(b): the sooner a result is produced the better but there is no deadline. We use a standard classification of real-time behavior which has three classes describing the effect of a missed deadline: soft, firm and hard real-time. With soft real-time systems, missing deadlines means results are less usable thus reducing the quality of service. Firm real-time systems allow the missing of deadlines but results are useless after the deadline and quality of service might suffer greatly. Hard real-time systems do not allow the missing of deadlines: one missed deadline means complete system failure with potentially catastrophic consequences.

Using the definition of real-time, we can define what a real-time application is:

Definition 1.2 In a real-time application, data is consumed and produced before a specified deadline expires.

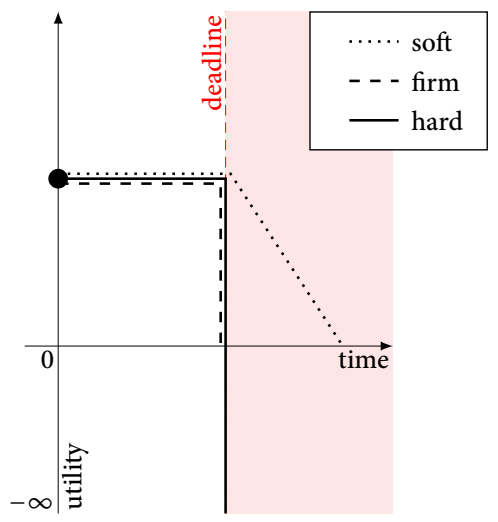

(a) For types of real-time systems

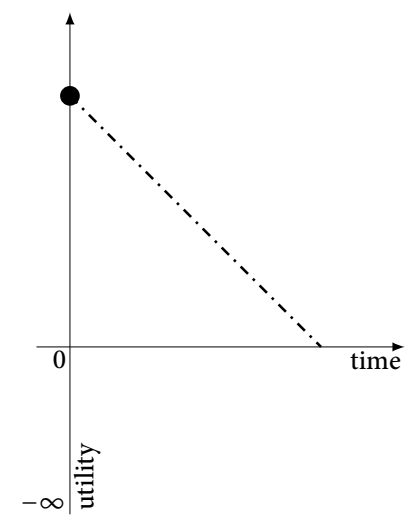

(b) For best-effort systems

FIGURE 1.1 - Utility vs time 
In this thesis our application domain for the developed architecture is firm realtime stream processing applications, such as are often found in SDR. Our focus lies on firm real-time systems where hard real-time techniques are used to evaluate worst-case scenarios which have to be feasible in order for the application to be considered admissible. This means that our architecture is designed to be predictable but contains components, such as the used SDRAM controller, for which no hard real-time guarantees are given; instead for some components like software functions estimates of Worst Case Execution Times (WCETs) are obtained by measuring. As such, these estimates can be optimistic.

Definition 1.3 A real-time system is a system, consisting of hardware and software, for which a model can be constructed which facilitates verification to establish that deadlines are met, and throughput and latency constraints are satisfied.

Note that Definition 1.3 only specifies that formal verification should be possible, in general it is of course desirable to have a model which facilitates verification with low effort. If we can derive accurate bounds on the temporal behavior we call the system predictable. We call a temporal analysis technique compositional if the global temporal behavior can be derived from the local temporal behavior of the components where all components are independently analyzable. A compositional analysis technique is required because it prevents that after global analysis the local analysis results must be updated and the complete analysis has to be repeated. A system with fixed priority arbiters requires an iterative analysis flow [86] and is therefore not compositional. This iterative analysis-flow has an exponential computational complexity and is therefore not efficient. Our objective is to define a real-time multiprocessor system for which efficient compositional analysis is possible.

The use of hard real-time verification methods simplifies the temporal analysis but results are only valid if the load hypothesis is satisfied [83], i.e. all tasks have an execution time smaller than the estimated upper bound that where obtained with measurements. Hard real-time techniques are applied because they simplify analysis considerably compared to probabilistic techniques. For probabilistic techniques such as POOSL [80] often a very large number of states need to be considered to derive the worst case. This is under the assumption that the input characteristics in terms of a probability mass function is an accurate representation of the behavior which is nearly impossible in practice [82].

The difference between the measured worst-case bound and the worst-case bound computed with a temporal model of a system is called the accuracy. Lower accuracy on the worst-case behavior of a system does not necessarily imply that such a system will have worse worst-case bounds than a system with higher accuracy. Allowing lower accuracy on the worst-case bounds in the behavior of a system results in a higher degree of freedom during the design of such a system and can result in potentially lower hardware costs. This concept is used in our work. 


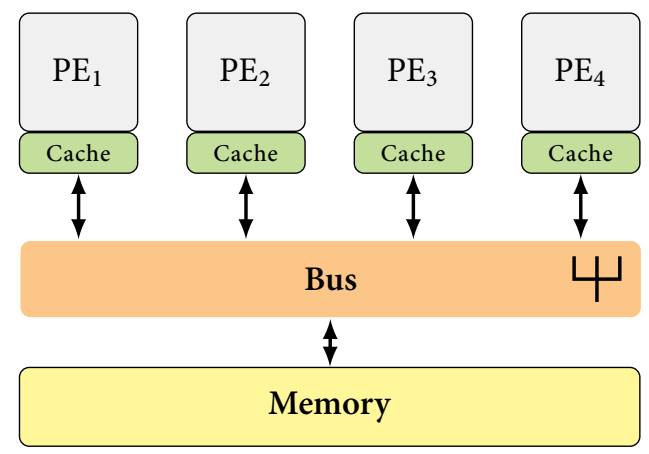

FIGURE 1.2 - Example of the SMP architecture class

\subsubsection{Classification of Architectures}

In this thesis we present a classification for multi-processor architectures to position our work. The first considered class is the Symmetric Multi Processor (SMP) architecture, as is shown in Figure 1.2. This architecture is an evolution of the classical single processor architecture as was used in early (personal) computers and is in essence a Von Neumann architecture. In this class of systems, a centralized shared memory is used by two or more processors. Often SMP architectures employ homogeneous processors running the same Operating System (OS). In this tightly coupled multiprocessor system peripherals are shared using a central interconnect, usually a bus, multi-layer bus or crossbar. Each processor can execute different programs independently and sharing of data and peripherals is usually managed by the OS. Often multiple levels of data and instruction caches are used to speed up processing and reduce the amount of concurrent access to shared resources like the main memory. Access to the main memory will have the same worst-case latency for every processor in an SMP architecture.

The often homogeneous nature of the SMP class architecture results in low efficiency for computationally intensive applications such as are used often in the SDR domain and the fact that the central interconnect and caches can become a bottleneck during program execution $[59,60]$. This will even become worse as the number of processors increases.

Task migration is usually supported and multi-threading allows a single program to use multiple processors. Architectures in the SMP class are optimized for besteffort applications which is also indicated by the use of superscalar processors and support for branch prediction. This makes the SMP class not very suitable for use in real-time embedded systems [61].

The second class of architectures we considered is the distributed architecture class which is similar to a Non-Uniform Memory Architecture (NUMA) architecture, as is shown in Figure 1.3. In this class, each processor has its own memory resulting in low-latency access to this memory for that processor. NUMA architectures are 


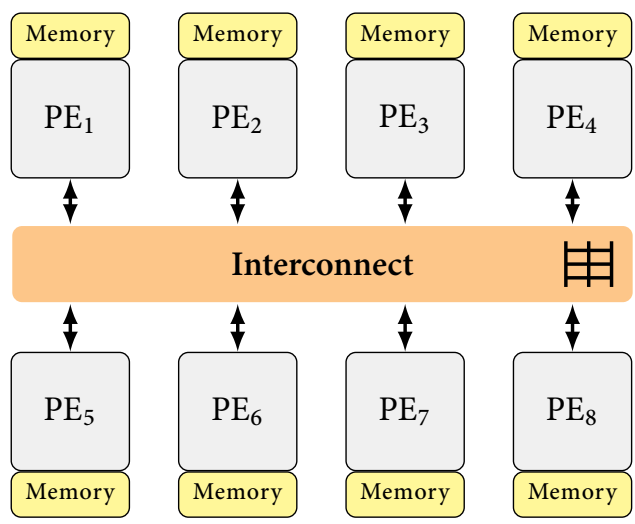

FIGURE 1.3 - NUMA class

more often heterogeneous than SMP architectures. Memories may or may not be remotely accessible and data exchange usually requires the use of a Direct Memory Access (DMA) controller. Examples of this class are the Kalray MPPA [41] and Recore Systems Many-core [63] architectures. Often the interconnects used (multilayer buses, crossbars or mesh-based topologies) are not very suitable for real-time use (only loose real-time guarantees can be provided) and tend to be expensive in terms of hardware costs. As memories are usually not shared between multiple processors there is little to no contention for access to the memory ports which can improve performance [8]. This potentially high performance resulting from using such a system comes at the cost of increasing the complexity of programming such a system $[10]$.

We can summarize the properties of the previous two classes as is shown in Table 1.2. When considering the domain of real-time stream processing applications we conclude that this type of application requires an architecture which supports both point-to-point data streaming and a shared memory to store large blocks of data or instructions. Data streams for stream processing accelerators should have a low synchronization overhead to allow cost-effective integration of small accelerators. As Networks-on-Chip (NoCs) providing integration support for stream processing accelerators are using crossbars or mesh-based topologies, they tend to have a high hardware cost. For example, the Æthereal [27] interconnect provides guaranteed throughput but its hardware costs are high [67]. A low-cost interconnect providing integration for stream processing accelerators and with support for guaranteed throughput is desired.

These desired properties are noted in Table 1.2. From this we can see that previously considered classes for multi-core architectures are not very suitable for real-time stream processing applications. As such, for real-time stream processing applications another class of multi-core architectures is desired. 


\begin{tabular}{rccc}
\hline & SMP & NUMA & SPA \\
\hline Scalability & - & + & + \\
Ease of programming & + & - & + \\
Heterogeneity & - & + & + \\
Suitable for real-time processing & - & + & + \\
Local memories & - & + & + \\
Central shared memory & + & - & + \\
Low-cost interconnect & $+/-$ & $+/-$ & + \\
\hline
\end{tabular}

TABLE 1.2 - Architecture classification overview (SPA = Stream Processing Architectures)

Another important aspect of the SMP and NUMA architectures is that they employ the classical master-slave protocol for communication. This type of communication is shown in Figure 1.4. For every transaction, there is a master and a slave where masters always initiates the transaction and slaves will wait for requests. For a data write, the master sends an address and a data word to write (1) and then waits for the acknowledgment that the write was accepted (2). A master performs a read by sending an address to the slave to read from (1) and waits for the slave to respond with the requested data (2). This means that a slave can not begin a transaction by itself and a master will have to wait during transactions for the slave to respond.

\subsection{Problem Statement}

Given the architecture classification presented in the previous chapter we distinguished that real-time stream processing applications require an architecture with properties which belong to both the SMP and NUMA class.

We observe that a low-cost architecture for stream processing applications could be very desirable. To keep programming effort low, a programming model suitable for stream processing applications is needed which additionally has to match with the desired architecture. As stream processing applications in the SDR domain will have real-time constraints, the desired architecture is required to be suitable for real-time stream processing applications. Cost-effective integration of stream processing accelerators will improve the performance of the desired architecture.

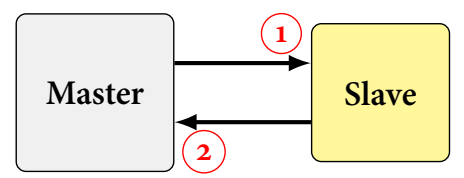

FIGURE 1.4 - Schematic overview of master-slave communication 
This leads to the following problem statement:

What key concepts are required for a realization of an architecture for low-cost real-time stream processing application which allows efficient compositional performance analysis?

More specifically:

1. On which concept can a low-cost real-time interconnect be based compared to existing NoCs and buses?

2. Which concepts can be applied to improve the computational performance of an architecture without increasing hardware costs significantly?

3. What concepts are needed to obtain independently analyzable components for a new architecture such that global temporal behavior can be derived from local temporal behavior of the components?

\subsection{Approach AND Contributions}

For real-time stream processing applications the principle of communicating tasks is typically used. As such, the proposed architecture should support point-to-point communication channels. In this thesis we present an interconnect with a ring shaped topology which implements a protocol for point-to-point streaming.

This protocol differs from the classical master-slave protocol as was described in Section 1.2.2. In the point-to-point protocol there are no masters and slaves, instead every Processing Element (PE) interacts with a FIFO communication channel. The PE sending data writes data into the FIFO buffer whenever there is space (1). After the write was accepted, the sending PE can continue processing (2). The PE receiving data reads data from the FIFO buffer whenever there is data available (1'). After the read returns data, the receiving PE can continue processing (2'). As such, in this protocol the sending PE does not have to send addresses to the receiving PE nor does the receiving PE have to reply with data to requests. In this fashion, the sender and receiver are uncoupled: as long as the FIFO buffer is not empty of full, both can continue processing without having to wait for each other.

Very low hardware costs are realized by sharing input buffers at the Network Interfaces (NIs) between communications and by using the concept of "guaranteed

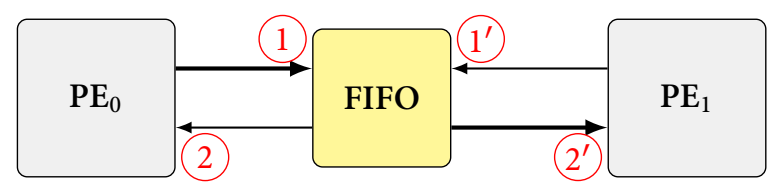

FIGURE 1.5 - Schematic overview of point-to-point communication 
acceptance" which allows us to omit buffers in the interface of the network and any logic for back-pressure. This means that memories connected to the ring are required to be able to accept data that is written to them from the network every cycle. Our bandwidth reservation mechanism for the network is work-conserving to improve the average-case throughput and latency without altering the worst-case bounds. We define work-conserving as:

Definition 1.4 A network is work-conserving when unused bandwidth reserved for streams can be claimed by another stream (under certain conditions).

The use of a predictable work-conserving bandwidth reservation mechanism enables the creation of a temporal analysis model. With this model real-time guarantees can be given.

While the concept of guaranteed acceptance is very suitable for dual-ported memories, it does not work for stream processing accelerators without some form of flow control. As our unidirectional ring has a very low hardware cost, we can provide cost-effective accelerator integration by combining two rings and applying creditbased flow control. In this concept, one unidirectional ring transports data for every peripheral while a second, smaller ring is used by a small shell in the NI to provide hardware flow-control for accelerators.

Additionally, accelerators can process data streams at a very fine granularity. As such, it is undesirable to perform coarse synchronization as this would result in the use of large buffers at the inputs of accelerators. The application of credit-based flow control in hardware allows for efficient synchronization at the word level.

Often not all processing capacity of stream processing accelerators is needed for the processing of a single data stream. Furthermore, functionality might be required multiple times within the same application or even multiple applications. This can result in high hardware costs due to duplicated accelerators with low utilization making this an undesired situation. However, the utilization of the hardware can be improved by multiplexing streams of data over an accelerator. As stream processing accelerators usually have internal state, multiplexing streams is not trivial to realize. A sharing approach for accelerators is needed for which an accurate temporal analysis model can be created. This model can be used to give real-time guarantees for each data stream which is multiplexed over a specific accelerator.

A stream processing application executing on such a platform can use the dualring interconnect to stream data from task to task. The instructions for all processors can be stored in a central memory and the use of sufficiently large caches will reduce the contention to the shared memory. Access to the central memory is implemented using a classical master-slave protocol and the worst-case latency to this memory is symmetrical for all PEs. As data and instructions are not necessarily stored in the same memory, the proposed architecture is not a Von Neumann machine. Temporal analysis models can be used to describe the communication channels in the proposed architecture. Therefore, it is possible to reason over the 


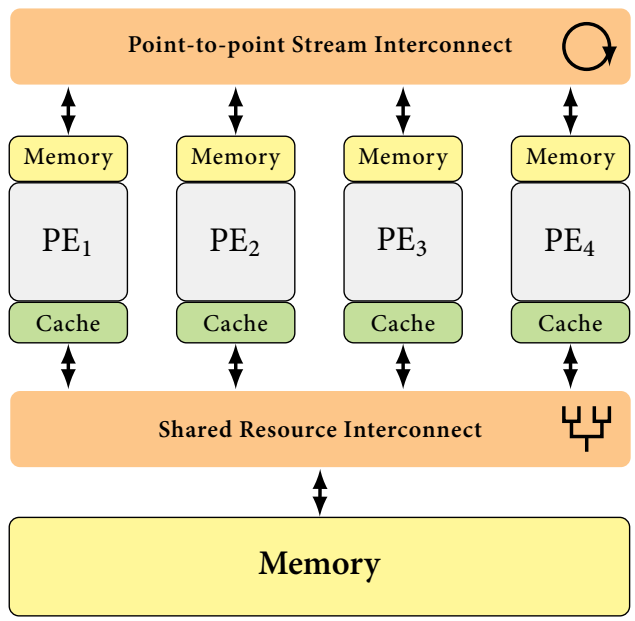

Figure 1.6 - SPA class

application performance under real-time constraints when the WCETs of each task is known, which was a requirement for real-time stream processing applications.

An abstract overview of the desired architecture is presented in Figure 1.6.

\subsubsection{Contributions}

The contributions described in this thesis are:

1. Introduction of a real-time heterogeneous stream processing architecture that allows efficient compositional performance analysis and supports both master-slave and streaming communication protocols such that stream processing accelerators and a shared memory is supported.

2. Introduction of a predictable low-cost communication interconnect with a ring-shaped topology.

3. The creation of a temporal analysis model for this interconnect in the form of a Synchronous Data Flow (SDF) model.

4. The creation of hardware flow control by introducing a second ring for oppositely directed flow control packets. This facilitates the integration of hardware stream processing accelerators that can not be used to implement flow control in software.

5. The creation of a temporal analysis model of the dual-ring interconnect in the form of a Cyclo-Static Data Flow (CSDF) model.

6. The development of an approach to share stream processing accelerators by different data streams under real-time constraints. 
7. The creation of a Synchronous Data Flow (SDF) model which enables the computation of the granularity at which blocks of data must be multiplexed over stream processing accelerators given real-time constraints.

8. The implementation and evaluation of instantiations of the proposed architecture on a Virtex 6 FPGA. For evaluation purposes a PAL video decoder has been developed given the specification of the PAL broadcast standard. Additionally, a stereo FM audio decoder and a GMSK decoder have been implemented.

\subsection{Structure}

The structure of this thesis is as follows. In Chapter 2 related work is presented on the various aspects of architectures for real-time stream processing. In Chapter 3 the proposed architecture is presented in a top-down manner. Chapter 4 describes the details of the unidirectional data ring for software-based stream communication and the accompanying formal temporal analysis model which is used to derive a hard real-time throughput bound. Chapter 5 will combine two unidirectional ring interconnects together with a small shell in each NI in order to support flow control which enables the integration of stream processing accelerators. The resulting combination of software and hardware-based FIFO communication is then analyzed using a temporal analysis model in order to determine the guaranteed throughput bounds. Chapter 6 introduces gateways as a solution to share stream processing accelerators between real-time data streams to improve their utilization. Chapter 7 evaluates the proposed architecture by means of three applications: a PAL video decoder, a stereo FM audio decoder and a GMSK decoder application. We present a summary and our conclusions in Chapter 8. 


\title{
2 \\ RELATED WORK
}

\begin{abstract}
The design and use of multi-core architectures is an active field of research. In this chapter we describe related work on multi-core architectures for Digital Signal Processing (DSP) applications as well as the type of interconnects used within such architectures.

We observe that traditional multi-layer buses are often replaced by Networkson-Chip (NoCs) with a mesh topology to reduce contention and to increase throughput. Both the multi-layer bus and its proposed replacements have a significant hardware cost which often scales superlinearly with the number of cores.
\end{abstract}

The proposed architecture will employ stream processing accelerators that have no notion of memory addresses. Therefore, the addition of such accelerators requires some form of interfacing before they can be integrated. Sharing of accelerators can improve utilization and improve flexibility of an architecture. Most approaches tend to be unsuitable for sharing under real-time constraints.

\subsection{INTRODUCTION}

In the previous chapter we described what we consider to be stream processing applications. The concept of communicating tasks presents the option of streaming data from $\mathrm{PE}$ to $\mathrm{PE}$, thereby removing the need to share data using a central memory. This implies that our proposed heterogeneous multi-core architecture should employ an interconnect which supports point-to-point communication.

Networks-on-Chip (NoCs) have been proposed as a replacement for traditional multi-layer buses. These NoCs often have a mesh-shaped topology and aim to reduce contention and increase throughput. Both the multi-layer bus and its proposed replacements have a significant hardware cost which often scales superlinearly with the number of cores. For multi-core architectures, this is a problem as with every generation more cores are added. 
In order to improve the performance at the same or reduced hardware costs, the proposed architecture will employ stream processing accelerators. As stream processing accelerators work on a stream of data, there is no notion of memory addresses. When applied in a memory-mapped multi-core system, some form of interfacing is required before accelerators can be integrated. Therefore, we will describe related work on the integration of accelerators in multi-core architectures.

The results from our case studies, as presented in Chapter 7, show that stream processing accelerators are often not fully utilized. At the same time, functional units such as filters are often used multiple times within the same signal processing application. To improve the flexibility concerning real-time applications that can run on a given multiprocessor system, to prevent the need for duplication of hardware blocks and to improve the utilization of existing stream processing accelerators, it is desirable to be able to process multiple data streams by the same accelerator while satisfying real-time constraints. In this chapter we present a number of sharing approaches for accelerators and conclude that for most approaches real-time guarantees are not provided.

The structure of this chapter is as follows. We will first present the definitions of properties used to classify networks in Section 2.2. A state-of-the-art overview of stream processing architectures is given in Section 2.3. Interconnects used in such architectures are explored further in Section 2.4. Related work on accelerator integration in memory-mapped systems is presented in Section 2.5. Sharing of accelerators under real-time constraints is discussed in Section 2.6. A summary of this chapter is presented in Section 2.7.

\subsection{BACKGROUND}

Before presenting related work for our architecture, we will first introduce some definitions that will be used to classify Networks-on-Chip (NoCs).

When considering on-chip interconnects for multi-core architectures, we distinguish two major classes: connection-oriented and connectionless interconnects. These are defined as follows.

Definition 2.1 We define an interconnect to be connection-oriented when it has separate connections between masters and slaves where properties can be specified for each individual connection [65].

Definition 2.2 We define an interconnect to be connectionless when it does not have separate connections between masters and slaves where properties can be specified for each individual connection.

In a connectionless network, communications are not separate and as such can influence each other which makes it hard to provide real-time guarantees [30]. 
Connection-oriented networks tend to have dedicated buffers per connection at the edges of the network while in connectionless networks buffers at the edge of the network can be shared.

In order for an interconnect to be suitable for use in safety critical systems, it need to supports Guaranteed Throughput (GT). We define this as:

Definition 2.3 Guaranteed Throughput (GT) is a property provided by the network for a communication channel which defines that a lower bound on throughput and an upper bound on latency is guaranteed [72].

In contrast to GT traffic, there is Best Effort $(B E)$ traffic:

Definition 2.4 Best Effort is a property provided by the network for a communication channel which defines that only the arrival order of the data is guaranteed but no bandwidth or timing guarantees are given.

We distinguish two switching policies that are used in NoCs: circuit-switched and packet-switched [70]. The first policy is commonly associated with for example buses and cross bars. Routers in the interconnect are set up to provide a dedicated channel for communication between endpoints [53,87]. While suitable for use in a real-time system, without detailed knowledge of communication within applications at design-time, contention for access to a circuit-switched link can cause the set up of a communication channel to fail.

The second and more actively studied [70] switching policy is packet switching. In these networks packets move from router to router based on routing information embedded into the data packet. While this type of network might share links for packets to different recipients, this sharing can also result in contention. Without detailed knowledge of communication within applications at design-time, it is impossible to determine the throughput.

In the next section we will present state-of-the-art multi-core architectures that can be used for stream processing applications.

\subsection{Modern Stream Processing Architectures}

Stream processing applications could perform better on an architecture that is designed for the concept of communicating tasks where point-to-point communication between tasks is explicit. We will now consider a number of state-of-the-art architectures for such applications. A comparison of these architectures is shown in Table 2.1. Each work is classified according to the architecture classification from Chapter 1.

The Chameleon [72] architecture is designed for stream processing applications. It combines a traditional multi-layer AMBA bus with either a packet-switched [42] 


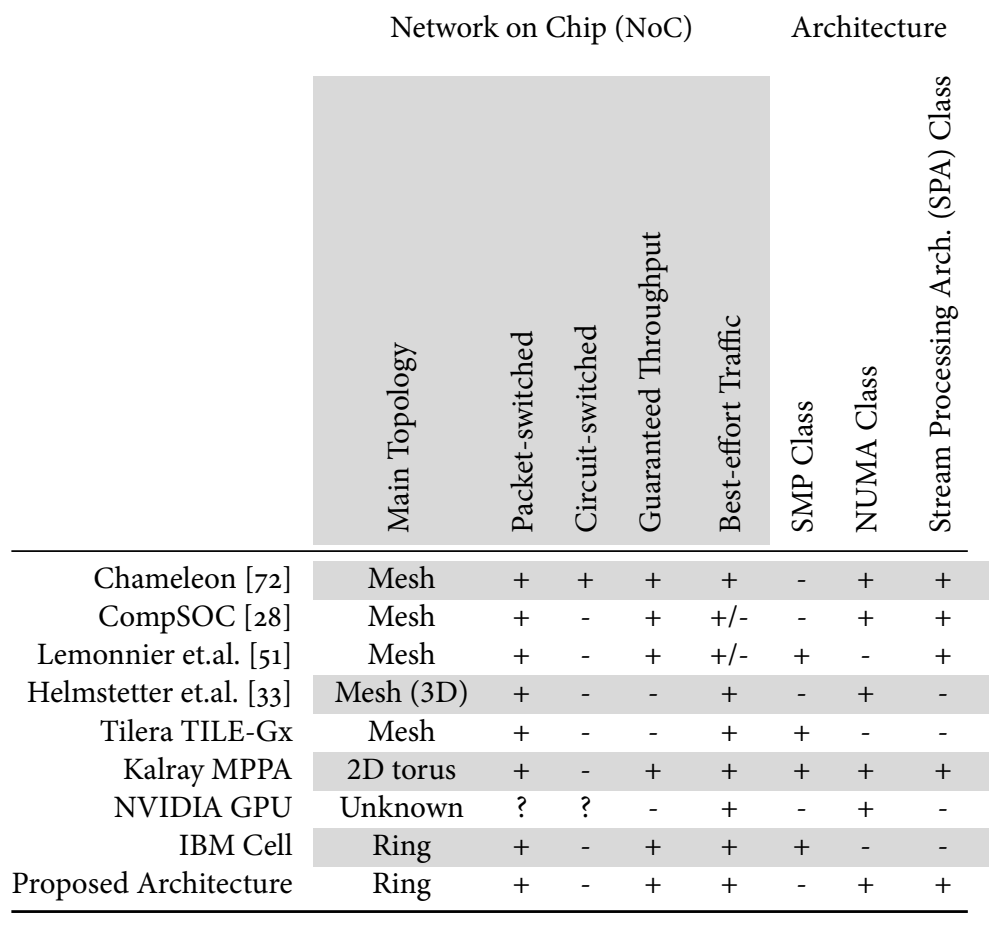

TABLE 2.1 - Comparison of architectures

or circuit-switched [87] NoC. The packet-switched interconnect from [42] uses a mesh topology. The circuit-switched interconnect from [87] uses a mesh topology with crossbars in the routers. The type of NoC can be selected at designtime and both interconnects support GT and BE traffic. The circuit-switched interconnect is more energy efficient as less arbitration is required compared to the packet-switched interconnect. However, every circuit-switched interconnect suffers from contention as the number of connections increases. Additionally, the circuit-switched interconnect from [72] is not work-conserving whereas the packetswitched interconnect is. While Guaranteed Throughput (GT) NoCs are used, no formal temporal analysis model is presented for this architecture.

CompSOC $[28,54]$ is an architecture for real-time stream processing applications. It presents virtualized instances of components such as processors to tasks. Each task executes using local memories. External memory is only indirectly addressable through the use of a DMA controller. As a result there is no need for processor caches. Applications are converted into dataflow graphs which can be analyzed to determine the minimum capacity requirements needed for the virtual components to obtain the required throughput. While it is claimed that Cyclo-Static Data Flow $(C S D F)$ can be used to describe applications, only the use of Homogeneous Synchronous Data Flow (HSDF) is demonstrated in publications. Processors and 
other components are shared by means of Time Division Multiplexing (TDM) between tasks. This strict virtualization provides "composability" where different applications can be composed to execute on the same architecture without the possibility of inter-application interference. This virtualization prevents the system to be work-conserving: if a task is not fully utilizing its virtual resources, another task can not use the free slack time. As such, CompSOC is not work-conserving, unlike our architecture. The Æthereal [27] interconnect or one of its variations is used which has high hardware costs [67] and provides point-to-point communication as well as access to shared components such as background memory. In contrast, our architecture realizes low hardware costs for the interconnect by using a small NoC for point-to-point communication and another small NoC for access to shared components. While the CompSOC architecture supports real-time stream processing applications, there is no support for the integration of stream processing accelerators.

In [51], a multi-core system is proposed for real-time applications. In this architecture, a multi-core system with numerous general purpose processors is augmented with an embedded FPGA solution to implement accelerators on demand and where needed. Accelerator interfaces connect instantiated accelerators to a NoC where a master-slave connection is implemented from a processor to an accelerator. This solution does not implement accelerator sharing but relies on adhoc instantiation. In the proposal from [51], there is no formal model to provide real-time guarantees. Additionally, they do not address the incurred delays of reconfiguring FPGA logic for task switching. Therefore, this architecture seems to target best-effort applications.

In [33] the P2012 architecture from STmicroelectronics is extended with a wrapper for the meshed-based NoC. This wrapper is designed for stream processing applications as it enables point-to-point data streaming for channels with dynamic behavior. While the authors present relative hardware savings compared to various implementations, the hardware costs of the meshed-based interconnect together with the wrappers for data streams are significant. Similar to our dual-ring interconnect in the proposed architecture, a credit-based handshake is used to provide hardware flow control. However, due to the underlying mesh-shaped interconnect, the hardware cost of the network in [33] is higher than our dual-ring interconnect. While the architecture from [33] targets stream processing applications, it only supports best-effort processing.

Multi-core architectures suitable for stream processing applications are also recently commercially introduced. Even so, none of these architectures target realtime stream processing applications.

Architectures like Tilera's TILE-Gx [77] family or Kalray's MPPA family of Multiple Processor Systems on Chip (MPSoCs) contain respectively up to 72 and 1024 processing cores. Both architectures provide a homogeneous multi-core platform with VLIW processors connected with an interconnect with a mesh topology. To ease the programming of so many cores, Kalray provides support for a "dataflow-style" 
programming where data streams are processed by tasks or kernels. This style of programming is very suitable for programs which contain task-level parallelism. The top-level program description which describes the communication channels between tasks is similar to the coordination language OIL. Both the OIL compiler and the Kalray compiler extract a dataflow graph for analysis. A key difference is that the OIL compiler can handle modal stream processing applications [24]. This dataflow graph, which is a form of CSDF in the Kalray compiler [14], is then used to perform mapping and allocation of communication channels [13], similar to what the OIL compiler does. However, for both architectures is it not shown that temporal guarantees can be given which is required for hard real-time applications.

Modern NVIDIA Graphical Processing Units (GPUs) like the G200 family and newer products are marketed as stream processing platforms [64]. These platforms perform operations on data streams in parallel using specialized languages which are in fact a type of coordination language. With every generation, the support for more complexity in these kernels increases but in essence, it remains a form of Single Instruction Multiple Data (SIMD). NVIDIA themselves like to refer to it as Single Instruction Multiple Threads (SIMT) as the processing cores use fast thread switching during I/O waits to improve performance. This sort of stream processing is very suitable for algorithms with lot of data parallelism [64] where massive amounts of data require the same treatment in parallel. This also implies that the operations have virtually no dependencies between streams as that would inhibit parallel execution. In this thesis we target stream processing in the SDR domain where data parallelism can occur but more often task-level parallelism is present. Additionally, while NVIDIA GPUs have a very high processing capacity, no techniques to derive temporal guarantees are provided which prevents the use of such platforms for hard real-time applications.

The Cell [38] processor from IBM uses an interconnect with a quad unidirectional ring topology. Data is transferred over the rings in counter-orientated pairs where burst transfers are required. The use of a ring network instead of a mesh or a crossbar was because of its low hardware footprint in combination with its "impressive throughput" [16]. No temporal analysis model has been developed for the ring network from the Cell processor. In contrast, our interconnect design is similar but allows us to describe the behavior of our interconnect by means of a temporal analysis model.

From these architectures, we can observe that networks are used with multiple topologies. In the next section we will present work on interconnects using various topologies.

\subsection{INTERCONNECTS}

In this section we discuss interconnects for multi-core architectures. We look into interconnects based on buses, mesh-topologies, crossbars and rings. We compare 


\begin{tabular}{|c|c|c|c|c|c|c|c|c|c|c|}
\hline & 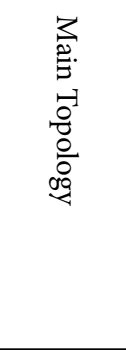 & 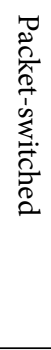 & 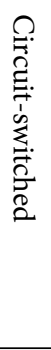 & 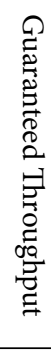 & 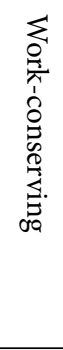 & 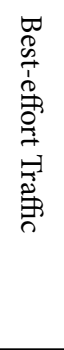 & $\begin{array}{l}z \\
z \\
0 \\
0 \\
0 \\
0 \\
0 \\
0 \\
0 \\
0 \\
0 \\
0 \\
0 \\
0 \\
0 \\
0 \\
0\end{array}$ & 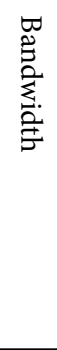 & $\begin{array}{l}\tilde{\hat{Z}} \\
\tilde{\tilde{\tilde{E}}} \\
\text { E. }\end{array}$ & 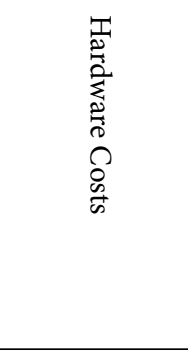 \\
\hline Single or multi-layer bus & Bus & - & + & - & & $+/-$ & + & $+/-$ & - & Medium \\
\hline Crossbar [45] & Crossbar & - & + & + & - & - & - & ++ & $+/-$ & High \\
\hline SoCBUS [53] & Mesh & - & + & - & & - & - & $+/-$ & - & Medium \\
\hline Wolkotte et al. [87] & Mesh & + & + & + & + & + & - & + & + & High \\
\hline Æthereal [25] & Mesh & + & - & + & $+/-$ & + & $+/-$ & + & $+/-$ & High \\
\hline dAElite [74] & Mesh & + & - & + & - & - & $+/-$ & + & + & Medium/High \\
\hline EIB from IBM Cell [44] & Ring & + & - & + & - & + & + & + & $+/-$ & Low \\
\hline Ring in Intel Nehalem CPU [46] & Ring & + & - & - & & + & $?$ & + & $+/-$ & Low \\
\hline Hierarchical Ring [18] & Ring & + & - & - & - & + & + & + & + & Low \\
\hline Our dual-ring interconnect & Ring & + & - & + & + & + & + & + & $+/-$ & Low \\
\hline
\end{tabular}


each interconnect with our ring network. An overview of the discussed interconnects is presented in Table 2.2.

Single-layer or multi-layer buses are commonly used in industry. Buses are a form of circuit-switched interconnects $[4,36]$. Compared to buses, our ring interconnect uses a small two-port multiplexer per connection instead of an $M$-port multiplexer per slave to select between $M$ masters. To support all-to-all communication, each multiplexer in a bus needs as many input ports as there are slaves, resulting in high resource usage and high wire count.

When a lot of bandwidth between masters and slaves is required and buses are no longer viable, a possible solution is to resort to using crossbars which are often fully connected, circuit-switch matrices [45]. This topology is usually constructed from a large number of multiplexers and therefore can also become quite expensive in terms of number of wires, power and area. This will result in a much higher resource usage compared to our ring network.

SoCBUS [53] is a solution which tries to combine the low and fixed latency of a bus with the flexibility of a mesh network. Along with relatively high hardware usage, SoCBUS locks a dedicated route through its mesh topology for each connection making it circuit-switched. This means that connections can fail to complete when all channels on a critical router are in use and as such there is always contention for capacity. Our ring provides automatic serialization due to its topology and as a result there is no contention within the network.

Another mesh-based network with virtual channels is presented by Wolkotte et al. [87]. This network can be used both in packet-switched and circuit-switched mode. While the circuit-switched version lowers hardware requirements because less buffering in routers is required, all packet decoding and flit routing still requires a significant amount of logic and buffers. In contrast, our ring network uses packets consisting of a single word and destination address which makes routing trivial and requires no address decoding.

Æthereal [25] is a packet-switched network which also employs a mesh topology where all buffering for GT traffic is done in the NIs. When used for BE traffic, buffering is needed in all routers. The network uses a precalculated TDM schedule to provide GT where both bandwidth and/or latency can be guaranteed. The amount of buffering required for each connection in the NIs results in more hardware compared to our ring.

Smaller and faster than Æthereal is its successor called dAElite [74]. In this version the support for BE traffic is removed which results in less buffers in the routers. The NIs still contain a buffer per connection. We will show that despite being connectionless, our packet-switched ring network can also support GT traffic. Unlike dAElite, our arbitration policy for the ring interconnect not only provides guarantees but makes it work-conserving as well. This means that we can use the interconnect whenever it has capacity available instead of just using a fixed transmission schedule. 


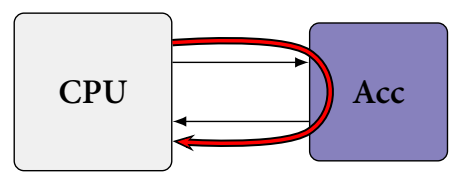

FIGURE 2.1 - An example of a co-processor

The use of a ring network as interconnect in a multi-core system is not new; it has been used in the Cell processor from IBM [44] and more recently by Intel in the Nehalem processor architecture [46]. The difference between our ring and existing implementations are the guarantees that our ring interconnect provides while keeping hardware costs low.

However, while a small ring results in low latency communication, increasing the ring size also increases the maximum latency. Increasing the latency usually is less of an issue in stream processing applications as the use of pipelining can hide most if not all latency. In case of a large design, the number of nodes might become too large for a single ring network. A potentially interesting solution is to use a hierarchical topology to promote locality while being able to service a large number of peripherals [18].

\subsection{ACCELERATOR INTEgration}

In a real-time architecture, the integration of stream processing accelerators in a memory-mapped architecture does not only impose requirements on the NoC. The used NoC also directly influences the method chosen for accelerator integration. In this section we present related work on accelerator interfacing for integration in an architecture.

We distinguish two separate approaches to accelerator integration. The first approach is the use of an accelerator as a co-processor. The second approach is the use of stream processing accelerators. We will now describe both approaches.

Co-processors process data dispatched by a master processor. As such, they are placed in the data path of the processor. Data is often transferred using a classical master-slave protocol. This concept is shown in Figure 2.1. The use of an accelerator as co-processor creates a latency-critical loop in the throughput of the processor where communication latency has a big impact. Normally, a processor must wait for data to be returned by the co-processor before the processor can advance. This means that the processor is idle when the co-processor is busy processing.

Stream processing accelerators are not placed in the data path of a processor but instead are placed between processors. This concept is shown in Figure 2.2. In our architecture this is facilitated by a protocol for point-to-point data streaming which is implemented in our dual-ring interconnect. The data stream originating from $\mathrm{CPU}_{0}$ is processed by an accelerator and the result is sent to $\mathrm{CPU}_{1}$. The 


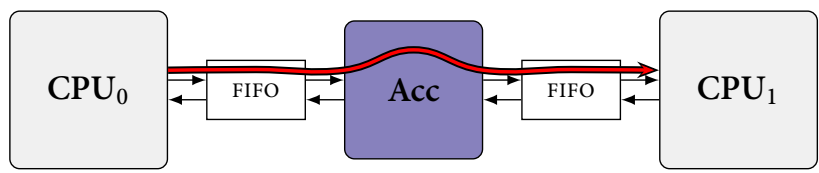

FIGURE 2.2 - An example of a stream processing accelerator

use of decoupling FIFOs for communication of the data stream allows the use of posted writes. This means $\mathrm{CPU}_{0}$ does not have to wait for the accelerator to accept data as long as there is space in the FIFO between the CPU and the accelerator. Additionally, since $\mathrm{CPU}_{0}$ does not have to wait for results from the accelerator, it can continue processing immediately after writing data to the decoupling FIFO, increasing its utilization. Another advantage is that communication latency will not influence throughput, unlike when co-processors are used.

In the next sections we will consider work on accelerator integration. We will first present work on integrating accelerators as co-processors. Work on stream processing accelerators is split into accelerator integration using interconnects supporting point-to-point streaming and work on integration by means of wrappers for accelerators to provide stream support.

\subsubsection{CO-PROCESSORS}

An example of a solution which integrates fine-grained accelerators in the data path of a programmable processor is the Imagine stream processor [43]. This processor employs a crossbar for the communication between accelerators and the Arithmetic Logic Units (ALUs). The accelerators and ALUs execute in a SIMD fashion under the control of a program counter. In contrast, the accelerators and processors in our design are data driven which introduces additional scheduling freedom compared to the tight centralized control from Imagine.

The concept of "conservation cores" [81] is similar to co-processors. In this work parts of a software algorithm are synthesized into dedicated hardware blocks which are called conservation cores. The software offloads work to these co-processors wherever possible in order to reduce energy consumption. As the master processor has to wait for the co-processor to complete before it can advance the program thread, this system is instruction driven. Because our design is data driven, once again we have the freedom to perform processing data in pipelined fashion whenever possible.

\subsubsection{Point-To-Point Streaming}

Interconnects with point-to-point streaming facilitate accelerator integration by simply removing the memory-mapped aspect of the architecture for the accelerator. Ideally, data streams are transferred using their own dedicated FIFO channel such as provided by the AXI4 Stream Interconnect [89] and Avalon Streaming Bus [3]. 
Both interconnects require design-time set up of point-to-point channels between peripherals which can not be changed at run-time making these interconnects inflexible. When communication patterns between components are not known at design-time, an interconnect with support for all-to-all connectivity is required which tends to result in high hardware costs.

Alternatively, circuit-switched interconnects like a single layer bus, cross-bar or interconnects supporting virtual channels $[25,53,74,87]$ could be used to integrate stream processing accelerators in an MPSoC design as long as the setup of the pointto-point channels is handled elsewhere. For real-time applications support for GT traffic is mandatory. Usually supporting all-to-all connectivity in such NoCs results in high hardware costs where this is supported by default in our low-cost dual-ring interconnect.

Contention in such NoCs can prevent channels from being set up when a critical router is in use. The automatic serialization of the ring topology coupled with our arbitration policy prevents contention and provides GT support. Streaming data support is realized by a shell in the NI of the dual-ring interconnect.

Rings have been used in commercial systems in recent years such as the Cell processor from IBM [44], the Intel Nehalem processor architecture [46] and more recently in the Maxeler dataflow computers [19]. The difference between our ring and existing implementations are the throughput and latency guarantees that our ring interconnect provides.

In [58], a ring network is introduced which proposes Rotating Time Division Multiplex Access (RTDMA) as a possible scheduling algorithm to divide bandwidth fairly between peripherals, which is similar to our arbitration policy. They support hardware flow-control but provide no back-pressure mechanism. Additionally, they do not support point-to-point data streams and are not work-conserving.

\subsubsection{WRAPPERS}

Instead of using an interconnect with support for data streams, a wrapper can be placed between a stream processing accelerator and an interconnect in order to provide stream support. The C-HEAP [56] FIFO protocol can be implemented in such a wrapper. However, these wrappers have a significant hardware cost as their functionality is comparable to that of a DMA controller. Our hardware flow control uses static or key-hole addresses for data and credit updates to prevent the need for dynamic address generation. By using C-HEAP only for software FIFO communication, we have maximum flexibility without high hardware costs.

A method to embed accelerators in a homogeneous memory-mapped system is presented for the STHORM architecture [9]. Both accelerators and their wrappers have a notion of memory addresses and as such require logic for dynamic memory address generation. The use of static addressing in our shells for each accelerator results in low hardware costs. 
In this thesis we implemented a small shell in the NI of the ring interconnect to provide point-to-point streaming for stream processing accelerators. This means our solution can be seen as both a very low-cost wrapper or an interconnect with low-cost run-time-configurable point-to-point streaming.

\subsection{ACCElERATOR SHARING}

In this section we present related work on accelerator sharing. The results from our case studies indicate that accelerators are often not fully utilized by a single data stream. At the same time specific functionality can be required multiple times in an application. Therefore, utilization of accelerators can be improved when accelerators can process multiple data streams.

Based on how they are interfaced in the integration in an architecture, sharing can be possible. In this section we will consider sharing for accelerators that have been integrated as an Instruction-Set Extensions (ISEs), by means of Remote Procedure Calls (RPCs) or by making use of DMAs. We additionally will describe architectures designed for accelerator integration which employ DMAs.

ISEs allow fine-grained interaction with application-specific accelerator hardware due to low communication latency and usually without synchronization overhead. This type of accelerator integration is applied in (general purpose) processors $[1,2,37]$ and proposed as a way to save power by integrating so-called conservation cores [81]. A potential drawback is that the processor is usually stalled until the accelerator returns the result which limits the maximum achievable performance gain. An additional drawback is that accelerators usually belong to a single core, limiting sharing of such accelerators to tasks executing on the same processor. Sharing between cores to improve utilization is not possible using this integration approach.

The use of RPCs [51] allows a less tight integration between processors and accelerators. For communication with accelerators synchronization needs to be added which results in some overhead. While concurrent processing of processor and accelerator is possible in theory, in practice it is difficult as results from the accelerator are often required for application progression. Additionally, it is not trivial to keep the processor occupied during the exact duration of the RPC call to maximize performance gains [71]. Effective sharing between cores requires some form of arbitration between all potential tasks that want to use an accelerator. When the number of cores is large, this arbitration tends to result in significant overhead.

An architecture which makes use of DMAs for the communication with accelerators is described in [78] and allows concurrent processing of data streams by processors and accelerators. In [78] they also describe a CSDF model for the derivation of the minimum throughput for the case that accelerators are shared by multiple streams. What is missing in [78] is the check for space at the output of a chain of accelerators, before processing of a block of data begins. Without this check, no correct CSDF model can be derived. Furthermore, determining an appropriate 
block size for every multiplexed stream given a throughput constraint has not been addressed in [78]. We will introduce a single actor Synchronous Data Flow (SDF) model for the derivation of the minimum block size which takes into account both the overhead caused by the flushing of data out of a pipeline of accelerators and the time needed for saving the state of the accelerators.

In [76] a real-time stream processing platform is introduced which supports stream multiplexing by providing each accelerator with multiple stream in- and outputs. Unlike our solution, in [76] state is stored within accelerators which allows for very fast context switches at the cost of having a limited number of streams that can be multiplexed. While the described platform targets hard real-time video processing applications, there is no form of formal analysis used. To increase the potential number of data streams that can be multiplexed, more memory has to be added to each accelerator to store state. In our sharing approach, state is stored in a single memory at the gateway. As a result, more flexibility is realized as state for all accelerators can be stored in one memory.

PROPHID [50] is an architecture which uses a crossbar with a precalculated TDM scheme to provide real-time guarantees. Accelerators stream data through FIFO channels, hiding all network aspects. Like our design, a special bus is used to configure accelerators while a streaming network handles all data. Processors in [50] are not connected to the data streaming interconnect, unlike our approach, making it impossible to integrate them in a stream processing application directly. Multiplexing of data streams is supported where state is retained within the accelerators, limiting the number of multiplexed streams per accelerator.

Eclipse [68] employs a shell to integrate stream processing accelerators, an approach similar to the dual-ring network used in our design. The use of the CFIFO [21] algorithm in a hardware shell results in larger hardware costs than the credit-based hardware flow-control support in our network. A shell around every accelerator manages arbitration between data streams. Our implementation only requires logic for arbitration in the gateways instead of in every accelerator. There is no temporal analysis model presented and performance is measured instead within a simulator.

Our approach of using a network to stream data between accelerators and a dedicated bus to save and restore accelerator state is similar to [6]. The interconnect from [6] provides real-time guarantees. However, the use of a switch to implement point-to-point connections results in higher hardware costs compared to the dualring interconnect which is presented in this thesis.

\subsection{Summary}

In this chapter we compared state-of-the-art stream processing architectures. These architectures contain a variety of interconnect topologies - some consisting of multiple topologies - and employ various approaches to program them. However, from all mentioned architectures only the CompSOC architecture is geared 
towards real-time applications using temporal analysis techniques. In this thesis we will present our architecture for stream processing applications. This architecture is constructed using components for which we provide dataflow models. These dataflow models are used for temporal analysis. The real-time bounds derived from these models for the proposed components can be used to provide real-time guarantees at the application level.

We presented related work on interconnects suitable for point-to-point streaming data as well as interconnects suitable for accelerator integration. The latter can be an extension of the first as stream processing accelerators usually have no notion of memory addresses and as such are not suitable for inclusion in a memory-mapped architecture without some form of interfacing support.

We observed that multi-layer buses are still often used despite their relatively high costs and drawbacks. These issues become worse when the number of connected peripherals grows. Mesh-shaped topologies or crossbars are proposed to replace buses but seem not often used in practice due to their high hardware costs. Lately ring-shaped topologies have been used as an alternative to buses in modern desktop processors and gaming consoles as their topology makes them a relatively lowcost replacement while still providing enough performance to be an attractive solution. However, these interconnect tend to have no explicit support for GT traffic.

In this thesis we present a dual-ring communication interconnect which has a low hardware cost and matches well with the concept of point-to-point communication as is used in stream processing applications. A lower bound on throughput and upper bound on latency can be guaranteed. This interconnect enables the integration of stream processing accelerators that can be shared between multiple data streams under real-time constraints. In the next chapter we will introduce the proposed architecture. 


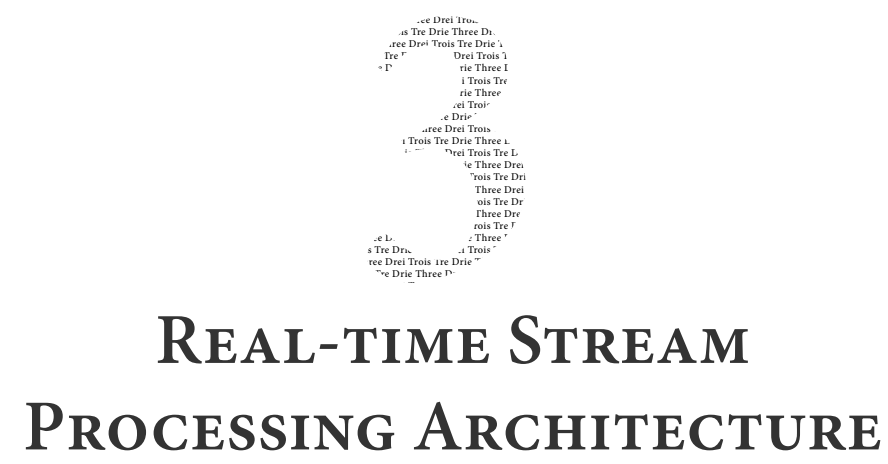

\begin{abstract}
In this chapter we introduce our real-time heterogeneous multicore architecture designed for the real-time stream processing domain. In this architecture concepts of the SMP and NUMA class architectures are applied. An interconnect with a ring topology is used for tile-to-tile communication and enables the integration of stream processing accelerators in a memory-mapped system. To improve the utilization of stream processing accelerators and to prevent the need for duplicated logic, accelerators can be shared between multiple data streams. This is enabled by the introduction of so-called gateway tiles for multiplexing data streams over accelerators under real-time constraints. A secondary tree-shaped interconnect is used to provide fair access to shared peripherals such as an external memory.
\end{abstract}

\title{
3.1 INTRODUCTION
}

In Chapter 1 we introduced a classification for multi-core architectures. We briefly describe the task-based programming style that we use for stream processing applications. In Chapter 2, aspects such as NoCs with various topologies and approaches for accelerators integration are described. In this chapter we introduce our heterogeneous MPSoC architecture for real-time stream processing applications. This architecture is designed to offer both the ease of programming for the SMP class of architectures with the scalability of NUMA class architectures in a design which matches with the concept of programs consisting of communicating tasks. This concept is supported in the proposed architecture by the introduction of a low-cost interconnect to provide all-to-all communication between all PEs. At the same time, the proposed architecture contains a large shared memory for example to store instructions.

In our architecture we call each PE a tile. Each tile contains the necessary periph- 
erals needed for that specific type of PE. For example, a tile containing a general purpose processor can also contain a local memory, instruction and data caches, a timer for interrupts and a local processor bus to connect these components. Communication with the rest of the architecture is facilitated by a memory-mapped bridge on such a tile. As another example, a tile containing a Finite Impulse Response (FIR) filter consists solely of a block of hardware implementing the filter, reading from an input and writing to an output connected to the rest of the system. Our architecture consist of numerous tiles connected by two interconnects: one for tile-to-tile communication and a second interconnect for master-slave communication such as is commonly used with components such as external memory. A schematic overview of the proposed architecture is depicted in Figure 3.1.

This chapter is structured as follows. In Section 3.2 an abstract overview of the architecture is given. The four different types of tiles are described in more detail in Section 3.3, Section 3.4 and Section 3.5. An overview of the dual-ring interconnect is presented in Section 3.6. The secondary interconnect used to share peripherals like external memory is described in Section 3.7. We provide a brief description how this architecture could be programmed using a top-level program description written in $\mathrm{C} / \mathrm{C}++$ or a coordination language such as OIL in Section 3.8. At the end of the chapter we summarize the described architecture.

\subsection{Architecture Overview}

The proposed tile-based architecture is oriented towards stream processing applications. This type of application consists of a set of communicating tasks and tends to be a mix of computationally intensive tasks and control oriented tasks. Often this means that the computationally intensive tasks contain little to no control logic but consist mostly of straight-forward arithmetical operations on data.

As such, application performance could improve significantly by mapping such tasks to a PE which is better suited: a stream processing accelerator. We define a stream processing accelerator as a configurable PE for a specific operation on a stream of data: for example a signal scaler or a FIR filter. When accelerators are implemented in a pipelined fashion, their throughput can be significantly higher than a similar function implemented on a general purpose processor. However, as stream processing accelerators operate on a stream of data, they have no notion of memory addresses and as such require some form of integration when applied in a memory-mapped multi-core architecture. A key difference between accelerators and programmable processors is that only processors have access to shared central memory to store large amounts of data. As such, accelerators are only suitable for applications that do not require to store large amounts of data. Most SDR applications belong to this class of applications.

Furthermore, we observe that while the use of pipelining in accelerators improves the maximum throughput, this processing capacity might not be utilized fully by a single data stream. The sharing of an accelerator by multiple data streams is there- 


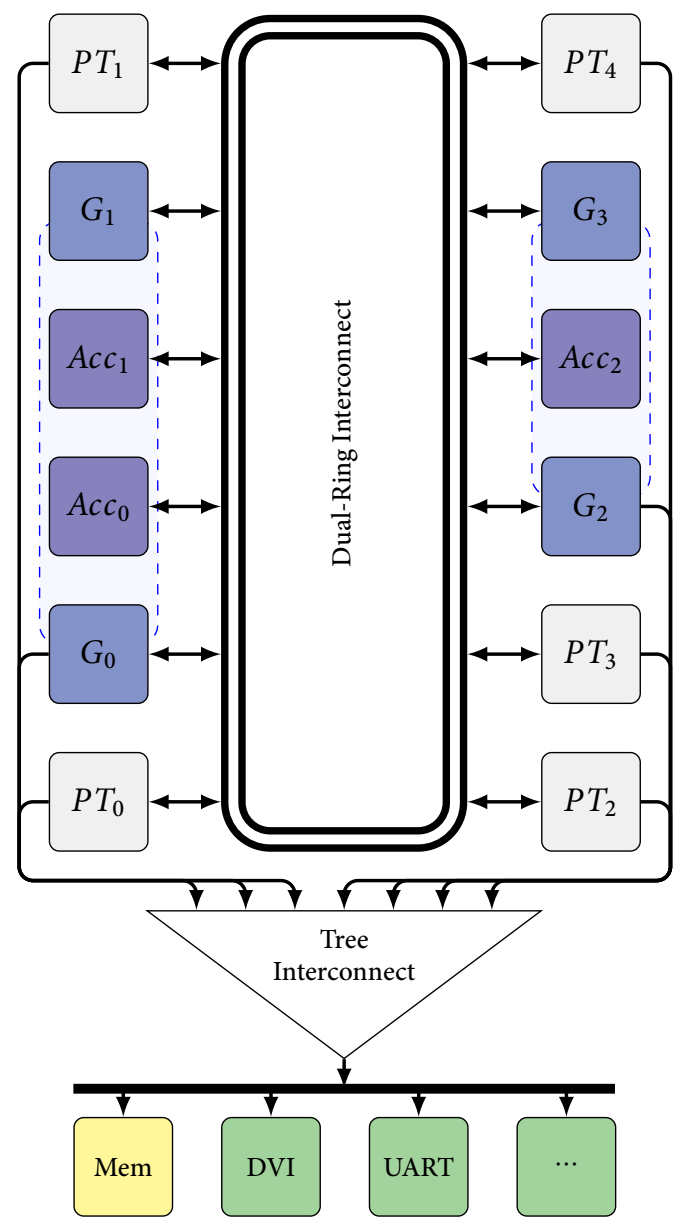

FIgURE 3.1 - Example architecture overview with all types of tiles

fore a desired property of the proposed architecture to improve the utilization of stream processing accelerators. At the same time this prevents the need to duplicate accelerators when a certain functionality is required by multiple data streams. Integrating stream processing accelerators and extending them with support for multiplexing multiple data streams will improve application throughput and lower hardware costs compared to a classical homogeneous approach.

\subsubsection{Tile-BAsed Architecture}

In our architecture a cluster of peripherals belonging to a PE (i.e. a processor or accelerator) is called a tile. We have four types of tiles in our design, each providing a different sort of PE. An abstract overview of our tile-based architecture is depicted 
in Figure 3.1.

The tiles come in four types: the "Processor Tile" (PT), which contains a processor and peripherals like local memories and a timer, the "Accelerator Tile" (Acc) which usually contains a single configurable stream accelerator, the "entry-gateway" tile (denoted by $G_{0} / G_{2}$ ) and the "exit-gateway" tile (denoted by $G_{1} / G_{3}$ ). To improve the utilization of stream processing accelerators and to prevent the need for duplicate logic in order to lower hardware costs, it is desirable to share stream processing accelerators between multiple streams. However, as most accelerators have internal state, this state has to be retained for each data stream when a context switch is performed. The granularity at which data streams are multiplexed is determined by the number of data samples in a block of data for a specific stream. This means that each data stream can use a different block size which remains constant for that specific stream. Multiplexing of data streams in blocks of data is performed by the gateway tiles. Each pair consisting of an entry- and exit-gateway handles multiplexing of data streams over a specific set of accelerator tiles. Such a pair of gateways is indicated by the dashed boxes in Figure 3.1.

Reconfiguring an accelerator or replacing state in accelerators while data is still being processed within those accelerators will result in corrupted data. Additionally, the time spent to process a complete block of data for a specific stream should have an upper bound to be able to provide real-time guarantees. As a result, one or more accelerators should be able to finish processing a block of data without introducing undefined (or even unbounded) waiting time because of back-pressure. This means that the output buffer at the last accelerator in such a chain should have sufficient space available to store the results of the complete block of input data before such a block can be scheduled for execution. If this would not be the case, it might be possible that the processing of a block of data will block the pipeline indefinitely, making it impossible for other streams to use the same accelerators, as is described in Section 6.3.7. To fulfill these requirements, the entry- and exit-gateway have to work together to ensure that both the pipeline is idle before another data stream is multiplexed and the output buffer at the last accelerator has enough space to store the resulting data. Only when these conditions are satisfied and when using an interconnect with temporal guarantees, hard real-time guarantees can be provided with the dataflow analysis that we apply.

\subsubsection{Communication}

In our architecture we use a low-cost dual-ring communication network to provide tile-to-tile communication. A fair bandwidth distribution algorithm is used to guarantee absence of starvation and allows us to determine a hard lower bound on the achievable throughput and an upper bound on the end-to-end latency for data packets traversing the ring. Integration for stream processing accelerators requires some form of fine-grained flow control as accelerators operate on streams of data and have no notion of memory addresses. Therefore, to integrate stream 


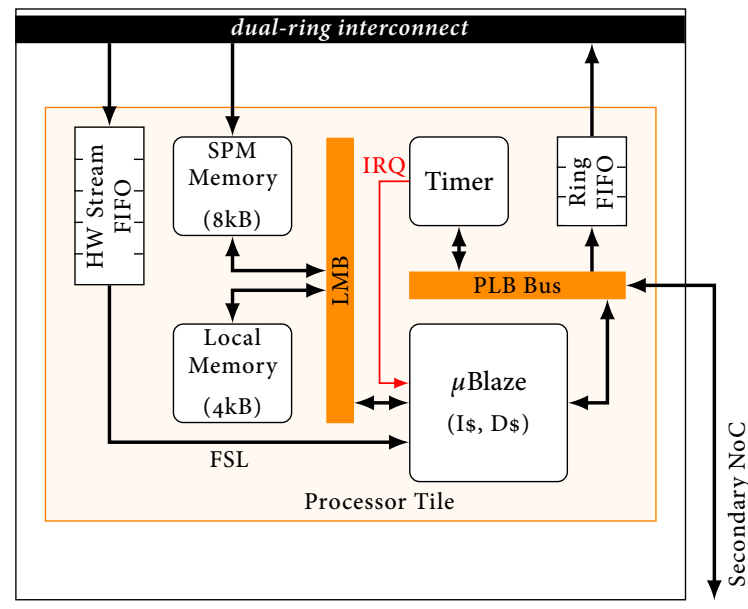

FIgURE 3.2 - Processor Tile

processing accelerators, hardware-based flow control is supported in our dual-ring communication network.

A second low-cost interconnect is used to connect each processor tile to a shared external memory and other peripherals [66]. This type of communication is masterslave oriented and is implemented as a binary tree interconnect. Each request is transmitted as a packet consisting of multiple flits. Arbitration between packets is done based on timestamps: at each router in the binary tree, the packet with the lowest timestamp is prioritized. This means that the amount of incurred latency has a worst-case upper bound and throughput has a worst-case lower bound.

In the next section we will introduce the first type of tile in our architecture: the processor tile.

\subsection{Processor Tile}

Figure 3.2 shows an overview of a processor tile. Every tile contains a RISC MicroBlaze processor and some peripherals like a timer for interrupts, a small local memory for a boot-loader and a small Scratchpad Memory (SPM) used to store more local data or buffers for the software-based C-FIFO communication. Every processor is equipped with an instruction and data cache which is sufficiently large ${ }^{1}$ to cache program code of tasks running on the processor. Tasks are governed by a realtime budget scheduler [75] from a POSIX compliant kernel with multi-threading support which was developed for this system [66]. The budget scheduler guarantees a minimum amount of time for each task to execute on the processor in a maximum interval of time.

\footnotetext{
${ }^{1}$ Architecture instances are used in our case studies with $32 \mathrm{kB}$ instruction and data caches
} 
Every processor tile is connected to a tree-shaped interconnect providing fair access to external memory, see Section 3.7. It also has a connection to a dual-ring interconnect providing streaming tile-to-tile communication for data streams. In order to keep hardware costs low, the inter-tile interconnect only supports posted writes: a write completes for a producer when the interconnect accepts, it does not wait until the write actually arrives at its destination memory.

The interconnect provides lossless communication where guaranteed acceptance (see definition Definition 3.1 on page 40) at every tile is required to prevent loss of data which removes the need for hardware flow control for communication with memories. Tiles can only write data to memories belonging to other tiles; when data from a remote tile is needed, both tiles need to work together to exchange data.

Processor tiles support two types of data streams: software FIFO communication, using the C-FIFO [21] algorithm which allows an arbitrary number of simultaneous streams between processor tiles, or hardware FIFO communication which is used to communicate directly with accelerator tiles. To support hardware FIFO communication we use a credit-based flow control mechanism. This is implemented with a second ring for the communication of credits in the opposite direction as the data. Currently it is only possible for a processor tile to receive one data stream from an accelerator tile and send one data stream to an accelerator tile to minimize hardware costs.

The hardware stream FIFO depicted in Figure 3.2 is used to receive a data stream from an accelerator tile, which is described in Section 3.4. This FIFO is connected directly to the MicroBlaze processor by means of a Fast Simplex Link (FSL) [88] connection allowing for single cycle access to the data stream.

\subsection{Accelerator Tile}

Accelerator tiles, as depicted in Figure 3.3, consist of a coarsely programmable accelerator and a streaming I/O port to the inter-tile dual-ring interconnect. The NI handles the transition from address-based communication to streaming data, which is explained in detail in Chapter 5.

In order for accelerators to be part of a real-time application, their WCETs should be provided. More complex accelerators are likely to use pipelined execution in order to be able to achieve high throughput rates. In our architecture, pipelined accelerators are required to produce data in a predictable manner. For example, for every data sample consumed, one output sample is produced where the worst-case temporal behavior of the stream processing accelerator is bounded by its worstcase latency and rate.

Accelerators have no notion of other aspects of the memory-mapped system: they consume a stream of incoming data from the NI and produce a stream of data which is returned to the NI. Accelerators can stall the incoming or outgoing stream 


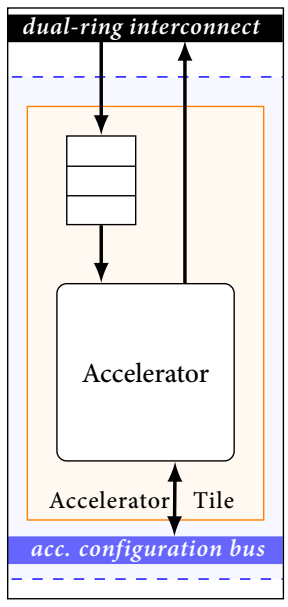

FIgURE 3.3 - Accelerator Tile

of data if they run out of data to process or buffer space to store results. To this end, the NIs of the dual-ring interconnect for accelerators use a credit-based flow control algorithm.

Each accelerator is connected to a bus to load and save its state and configuration. As such, accelerators which are to support multiplexing of multiple data streams are required to have logic to load and restore internal state. This is used to provide context switches when different data streams are multiplexed.

Accelerators are chained together at run-time by a description written by a programmer which describes the flow of data between tiles. A support library abstracts the implementation details and allows a programmer to simply connect blocks of functionality in a programming language like $\mathrm{C} / \mathrm{C}++$ or a coordination language like OIL.

\subsection{Gateway Tiles}

In our architecture we have two types of gateways: entry-gateways and exitgateways. Entry-gateways are based on processor tiles and as such contain mostly the same components plus some extra components needed for managing accelerators and multiplexing data streams, as is shown in Figure 3.4. Configuration and state of accelerators are loaded and saved by means of an accelerator configuration bus which is connected to all managed accelerators. Configuration and state are stored in the local memory of the entry-gateway for every multiplexed stream. To prevent the speed of the processor becoming a bottleneck in the data path, data is forwarded to the accelerators by a small DMA.

The entry-gateways handle streams using a Round-Robin $(R R)$ scheduling policy. 


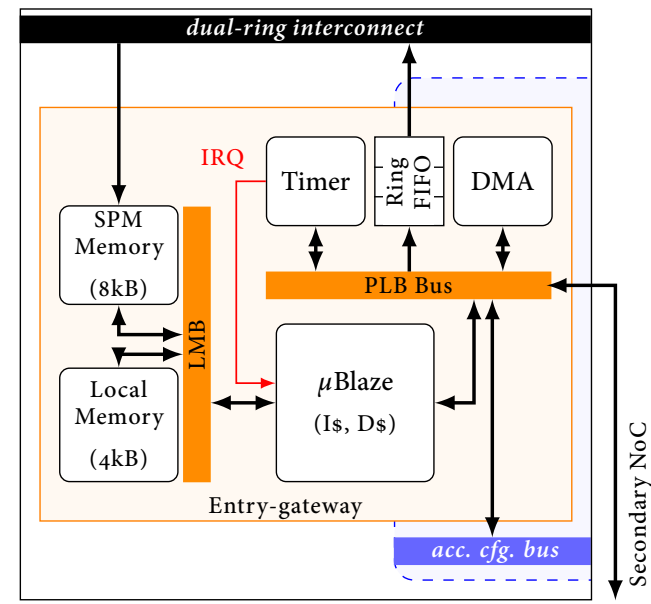

FIGURE 3.4 - Entry-gateway

The use of RR allows multiplexing of unrelated streams for multiple applications that are being executed simultaneously on the MPSoC.

Data streams arriving at the entry-gateway use software FIFOs to store their data in the dual ported C-FIFO memory. The DMA controller copies data for the currently active stream from this local FIFO memory to an accelerator using hardware creditbased flow control. Produced data by the last accelerator in the chain is passed to the exit-gateway, which converts from hardware to software flow control, to a processor tile or entry-gateway.

The exit-gateway is an accelerator containing a DMA converting the hardware flow controlled data stream to the address-based software flow controlled FIFO, as is shown in Figure 3.5. When the last sample from a data block passed through the exit-gateway, the entry-gateway is notified that the pipeline is idle and another data stream can be multiplexed.

\subsection{DUAL-RING INTERCONNECT}

In our architecture for real-time stream processing applications, an interconnect is used which provides point-to-point data streaming between processor tiles and accelerator tiles. This network is designed to have low hardware costs compared to most alternative topologies like multi-layer buses or mesh networks, provide fair arbitration between all traffic sources and has to be predictable in order to allow the derivation of a dataflow model used for temporal analysis.

Our dual-ring interconnect combines two unidirectional rings to support software and hardware-based flow control. By applying hardware flow control only for data streams involving accelerators, we keep hardware costs low while the use of soft- 


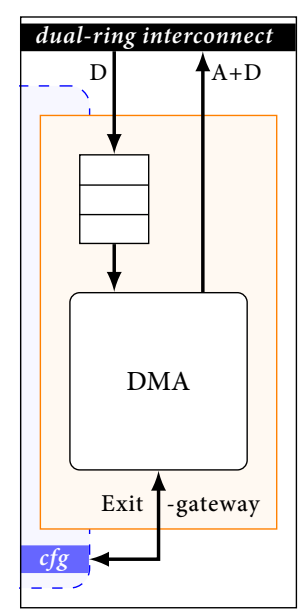

FIgURE 3.5 - Exit-gateway

ware flow control between software tasks maximizes flexibility in communication patterns.

\subsubsection{Single Ring Design}

In our architecture, we have multiple tiles connected to a dual-ring interconnect consisting of two unidirectional rings. In this section we explain the global structure of such a unidirectional ring, for a detailed description see Chapter 4 . The ring interconnect allows tile-to-tile communication and synchronization for processors using our software FIFO channels (see Section 3.6.2).

It is designed with the following requirements:

» Fair arbitration for all traffic sources resulting in a guaranteed minimum throughput for every source

"Work-conserving to improve the average packet latency

»Scale linearly with the number of connected tiles

"Decentralized arbitration to improve scalability and prevent the need for long wires for high performance

Our ring design is based on the design from [66] which was extended with a new packet arbitration algorithm which we call "ring slotting", see Section 4.3. In this arbitration algorithm, the passing of data from one NI to the next is considered the passing of a unique container which is numbered and contains an address and a data word. Additionally, each NI has its own unique number. By only allowing NIs to use their own slot - the slot which has the same number as the NI - every tile on the ring is guaranteed to have a available slot to transmit data every time 


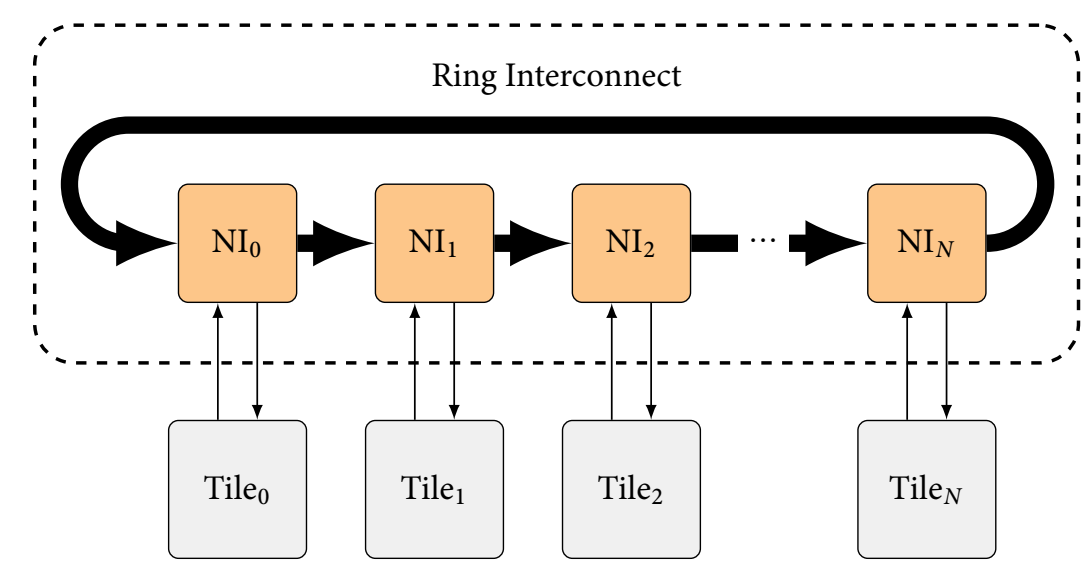

FIGURE 3.6 - Single ring design consisting of chained ring Network Interfaces

the slots do a full revolution. The arbitration is work-conserving by allowing NIs to inject traffic into an empty slot when this slot will not reach its owner before the destination NI for the data is reached. This algorithm is explained in more detail in Section 4.3.

The arbitration algorithm we designed can be implemented at a low hardware cost as slot numbers are fixed at design-time. This is an implementation choice; other bandwidth distributions can be realized using different numberings but this will affect the corresponding temporal analysis model. The implementation of the arbitration results in hardware consisting of only comparators and simple logic gates, resulting in fast logic with a small footprint.

We do not distinguish between routers and NIs in our ring; a ring topology is formed by chaining a number of NIs together. See Figure 3.6 for an abstract example of this design.

Processor tiles contain a dual ported memory to receive incoming data which is connected to the local output port of a ring NI. In this design, data is written directly into the dual ported memory based on the destination address accompanying each data word. The address used for writing data describes a destination NI to deliver the data to as well as an address within the destination tile.

The need for buffers between NIs and flow control within the ring can be prevented by requiring that every tile adheres to "guaranteed acceptance".

Definition 3.1 "Guaranteed Acceptance" means that data presented at the local output of a NI has to be accepted by the tile in a single cycle.

All used on-tile memories are capable of single cycle reads and writes and as such adhere to concept of guaranteed acceptance. The use of software-based flow con- 
trol on top of the unidirectional ring to implement FIFO channels using ScratchPad Memories (SPMs) allows tasks implemented in software to stream data between them. However, stream processing accelerators do not use an SPM but instead have a small FIFO buffer; in order to integrate these into the architecture, the single ring design does not suffice.

\subsubsection{Software Flow Control}

In this section we describe the software algorithm which implements a FIFO channel with distributed pointers using local and remote SPMs on Processor Tiles.

Despite only supporting writes to remote memories our ring network can be used for a distributed FIFO implementation, based on C-HEAP [56] which we call CFIFO. C-FIFO provides support for back-pressure at the application level using only one ring of the dual-ring interconnect. These FIFOs are used for the communication between parallel tasks in stream processing applications that are described by task graphs.

The FIFO uses data containers which are an arbitrary number of words in size and works by means of distributing read and write pointers. These pointers are used in a Round-Robin (RR) fashion on the containers of the FIFO instance. At the FIFO input the local write pointer is compared with the read pointer to determine whether there is space to write data. When the pointers are the same, a special bit on both pointers, called a wrap flag [56], is used to distinguish between an empty and a full FIFO. This wrap flag is toggled every time a pointer rolls over: when the pointers and their wrap flags are identical the FIFO is empty, otherwise its full. The C-HEAP algorithm only requires support for ordered writes: it does not require locks, mutexes or atomic read-modify-write support.

The task writing into the FIFO buffer (the writer) has a copy of the read pointer which is managed by the task reading from the FIFO: it is only updated by the "remote" task which acts as the reader. See Figure 3.8(b) for an example of writing a container into a FIFO between two tasks running on two different cores. Whenever data is written, it is sent to the allocated FIFO memory at the remote peripheral (1). After the data is written, the write pointer is updated (2) and the update is written to the remote peripheral as well (3). Note that posted writes to a remote memory are always guaranteed to be received in the same order as they were issued; as such the pointers are always modified after the data is written. Our hardware supports streaming memory consistency [79] which guarantees that reads and writes from a specific processor to the same memory arrive in the same order that they were issued.

At the reader - the task that reads from the FIFO buffer - the copy of the write pointer is compared to the read pointer to determine if data is available. If this is the case (the read and write pointer differ), data is already stored in the local data memory of the peripheral. As such, reads from the FIFO are always from local memory which also means every read is performed in a single cycle. 
See Figure 3.9(c) for an example of reading a container from a FIFO. After a data container is consumed from the FIFO (1), the read pointer is updated to reflect this (2). The read pointer is then written to the memory of the remote peripheral (3) to update the state of the FIFO.

The use of C-FIFO allows every processor tile to use multiple software FIFO channels simultaneously to other processor tiles or gateway tiles. The overhead resulting from having to compute and update two pointers every read or write of a container to the software FIFO means that C-FIFO is suitable for coarse-grained communication. By using data containers that are much larger than the size of the pointers that have to be updated, the relative overhead is reduced.

Not only do stream processing accelerators require flow control in their input and output channels, stream processing accelerators can usually process data at a very fine granularity. Thus fine-grained synchronization is required which makes the use of the C-FIFO algorithm less attractive.

\subsubsection{Hardware Flow Control}

The single ring design works excellent for software-based FIFO communication but the lack of flow control, which resulted in low hardware costs, makes it unsuitable when integrating stream processing accelerators. In order to facilitate this, stream processing accelerators need a form of flow control.

We combine two unidirectional rings as described in Section 3.6.1 where one ring is used to transport data between tiles and the other is used for flow control for stream processing accelerators. A small shell at each NI implements a credit-based handshake [47] for flow control by combining these two rings into a single dualring interconnect. The second ring is used to transfer credits that are only one bit wide - in contrast to the 32-bit data words used on the other ring - resulting in lower hardware costs for the second ring compared to the first.

By implementing hardware-based flow control only for stream processing accelerators, it is possible to transfer streaming data at a very fine granularity - down to a single data word - which implies that very small buffers can be used at the inputs of accelerators. Data is exchanged using data containers which have a defined capacity of $D$ data words, which can differ per stream ${ }^{2}$. The depth of these buffers is denoted in the flow control algorithm with a corresponding number of credits. As long as the shell at the producing accelerator has credits, it consumes one of these credits each time a data word is sent to the shell at the receiving stream processing accelerator. When data at the input of an accelerator is consumed, a credit is sent to the shell at the data producing accelerator using the second ring to indicate that a buffer position is free.

The use of two oppositely-oriented rings keeps the latency between two communicating stream processing accelerators balanced: data travels the same number of

\footnotetext{
${ }^{2}$ In our current implementation the size of these data containers is fixed at design-time
} 

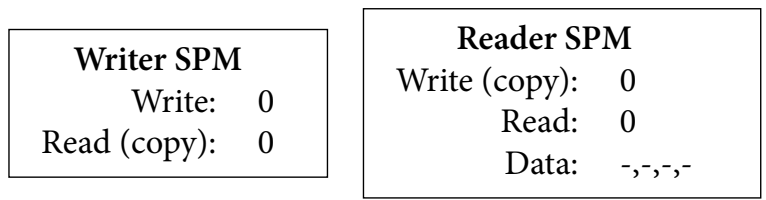

(a) Empty FIFO

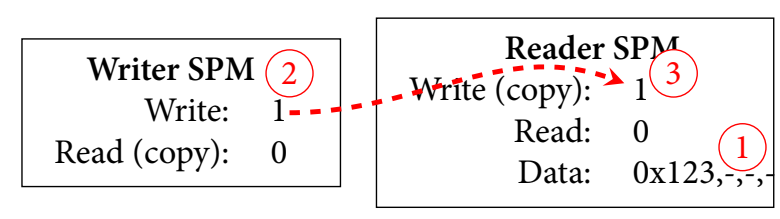

(b) Write data to remote memory, update local write pointer and update remote write pointer

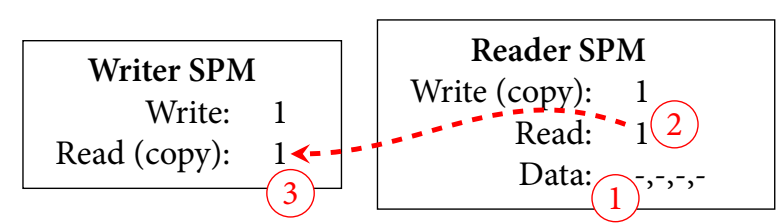

(c) Read from local memory, update local read pointer and update remote read pointer

FIGURE 3.10 - Examples for a distributed FIFO with capacity 3

hops as the credits. This means that when processing is kept local, i.e. by using accelerators which are only a couple of hops from each other, low latency communication is possible.

A more detailed description and derivation of a temporal dataflow analysis model is presented in Chapter 5 .

\subsection{Secondary Interconnect for Shared Slaves}

In the architecture overview from Figure 3.1 a secondary low-cost tree-shaped interconnect [66] is shown which provides master-slave access to shared components such as an external memory and DVI video output. In this interconnect, a binary tree-shape topology is used to route traffic from masters (tiles) to slaves.

This interconnect was designed to have the following requirements [66]: 
» fair scheduling to ensure liveness of all cores

» work-conserving to optimize for average read latency

" scale linearly in hardware costs compared to the number of tiles connected

" pipelined and decentralized arbitration to avoid long wires to obtain high performance

Every read or write request is split up into multiple packets called flits and transmitted with a command, destination, return address and a time stamp upon entering the tree. This time stamp is used for First-Come-First-Served (FCFS) arbitration: when two flits arrive at the same time at a router, the packet with the oldest (highest) time stamp is serviced first. As such, arbitration is done per packet and not per flit. Time stamps are generated at each router and are synchronized during reset. As long as all routers generate time stamps synchronously, the FCFS arbitration is fair. The number of bits used in timestamps depends on how much clock cycles difference there can be between two packets that are in the tree simultaneously. At the root of the tree, a multiplexer is used to select the slave for which a command is received.

The tree interconnect has back-pressure-based flow control for commands sent to slaves as a response might take multiple clock cycles. Reply packets from slaves do not require any arbitration or back-pressure as the NIs at the masters are always ready to accept data for outstanding commands. This reduces the complexity of the return path and as such lowers hardware costs.

\subsubsection{Shared Memory}

One of these slaves is a central shared memory as is also used in SMP architectures. This shared memory can be used to store a single binary program to be executed by all processors in the system. As explained in Section 3.3, processors can be fitted with a data and instruction cache to reduce the amount of traffic to the shared memory. The kernel state and stack is located in local memory, FIFO data is stored in a local SPM; only data stored in the heap is stored in shared external memory.

\subsubsection{Other Peripherals}

Other slaves available in our implementation are those available on the used development board: a Xilinx ML-605 sporting a Virtex 6 FPGA. These include a serial port, LCD panel, DIP switches, buttons and a number of LEDs.

In our case study of the PAL video decoder we use a DVI output to display the resulting video on a monitor. The control interface of this DVI controller is connected as a shared slave to this tree-shaped interconnect. 


\subsection{Programming}

Programming this system is facilitated by means of a program description written in the OIL coordination language or in $\mathrm{C} / \mathrm{C}++$. When using plain $\mathrm{C} / \mathrm{C}++$, the programmer has to perform the mapping of tasks to processing elements, set up communication channels between communicating tasks and determine the depth of the buffers used in those communication channels. This is a tedious and error prone process and as such is not desired. Instead, the set up of communication channels and allocation of tasks to cores can be generated from a program description using a coordination language such as OIL.

An OIL program can be compiled by the Omphale compiler. This compiler can compute the minimum buffer sizes needed to guarantee absence of deadlock and perform mapping of tasks to PEs and compute minimum buffer sizes in order to obtain a specified minimum throughput. As the compiler handles the mapping of tasks, it automatically generates the code required to set up all communication channels.

Software tasks contain one or more functions that are usually described in $\mathrm{C} / \mathrm{C}++^{3}$ and the only restriction imposed by our programming model is the requirement that a function is side effect free.

Definition 3.2 A function is side effect free when an execution of this function will only affect the local state belonging to this function: the global state or state belonging to other functions is never modified by the execution of this function ${ }^{4}$

This means that a task can not perform hidden state manipulation: it can not update memory addresses belonging to the global state or other functions. However, it is possible to have internal state within a function: for example a FIR filter will retain the coefficients and other state variables between invocations of the function. As such, it matters in which order the data is passed through such functions; reordering input data can yield corrupt results. The requirement for side-effect free tasks results in the possibility to map tasks on any suitable processing element as inter-task dependencies are explicitly described as interconnected in- and outputs in the top-level program description.

When a task only uses local variables and reads and writes to data structures belonging to the FIFO communication channels, only locally available memories will be used. As such, advanced knowledge of the memory map per PE is not required at design-time.

When large data segments have to be shared, dynamically allocated memory is needed using the shared central memory. While dynamic memory allocation can

\footnotetext{
${ }^{3}$ The use of the GNU Compiler Collection (GCC) allows software tasks to be implemented in any supported language - in this thesis we have used $\mathrm{C} / \mathrm{C}++$ in all our case studies

${ }^{4}$ This differs from (purely) functional programming where a function can not modify global state or retain internal state after it completes
} 
also be used for local memories, data can not be shared between tiles as such memory segments are not readable from other tiles. Each processor has a cache for the shared memory to reduce contention but there is no hardware cache coherency as this tends to result in high hardware costs. Instead, software cache coherency [66] is implemented.

When writing programs for safety critical real-time systems, the use of dynamically allocated memory from within software tasks makes it impossible to derive an upper bound on the required memory for the application. Aside from using malloc () and similar functions, another form of dynamic memory allocation is recursion: if a task does recursive calls to a function based on its inputs, in general no upper memory bound can be given.

As such, the following rule is defined:

Rule 3.1 In order to limit the amount of memory used, the use of dynamic memory allocation or recursion is prohibited from within a software task

Using Rule 3.1 we can guarantee that a software task always uses an at design-time known amount of memory which allows us to guarantee an upper bound for the total amount of memory required for all tasks within an application.

Computing elements without a processor (e.g. accelerators) need some form of initial set up in order for an application to begin execution. Currently, extensions of the Omphale compiler are in development to process mapping hints to describe which tasks are to be executed in software and which tasks could be executed on stream processing accelerators. At the time of writing, the programmer needs to set up the use and sharing of a stream processing accelerator using a support library which abstracts the use of shared stream processing accelerators to a simple interface which connects inputs and outputs.

After compiling the source code, the resulting binary executable is placed at one location in shared memory for all processors. Every processor runs an instance of its own POSIX compliant real-time OS [66] and executes the same program; the internal core identifier is used to determine which subroutine is to be executed on a specific core.

\subsection{SUMmary}

In this chapter we introduced our heterogeneous multi-core architecture for realtime stream processing applications. PEs are grouped into tiles which are interconnected by two networks: our dual-ring interconnect provides real-time pointto-point data streaming while a tree-shaped interconnect provides fair access to slaves such as an external memory and other shared components.

In our architecture we currently have four tile types: processor tiles, accelerator tiles, entry- and exit-gateway tiles. Processor tiles use a software-based FIFO 
communication channel and accelerator tiles use credit-based flow control implemented in hardware in the shells of the NIs. This provides low-cost communication between software tasks with relatively low overhead when using coarse-grained communication. Smaller buffers can be used at accelerator tiles compared to processor tiles due to fine-grained data streaming with low synchronization overhead resulting from the hardware flow control.

The resulting architecture is intended for stream processing applications which can have a top-level description using a coordination language such as OIL. The use of a top-level description to explicitly describe the communication between tasks abstracts the use of the underlying hardware from a programmers perspective. This simplifies the development of programs for our real-time multi-core stream processing architecture. 


\title{
4 \\ LOW-COST RING INTERCONNECT
}

\begin{abstract}
Aвstract - Connection-oriented guaranteed-throughput mesh-based Networks-on-Chip (NoCs) have been proposed as a replacement for buses in real-time embedded multiprocessor systems such as used for Software Defined Radios (SDRs). However, even with attractive features like throughput and latency guarantees they are not always used because their hardware cost tends to be higher than buses.

In this chapter we present a communication ring that provides guarantees for minimum throughput and maximum latency. This ring is an attractive communication network as replacement for buses for small to medium scale embedded multiprocessor systems for real-time stream processing because of its relatively low hardware cost. Additionally, all-to-all communication can be used for point-to-point streaming without the need for shared memory which matches with the stream processing paradigm.
\end{abstract}

We show that the data serialization of our ring makes it contention free and enables sharing of buffers which reduces the hardware cost. A further cost reduction is achieved by implementing end-to-end flow control in software and by supporting only writes over the network. Dataflow analysis techniques are used to prove that throughput and latency guarantees can be given despite that the proposed communication ring is connectionless.

\subsection{INTRODUCTION}

When an MPSoC for real-time processing is used for SDR applications, the used NoC should ideally be able to provide guarantees at the application level. As defined in Definition 2.1, we distinguish between connection-oriented networks and connectionless networks. Connection-oriented networks tend to have dedicated

This chapter is based on work presented in [BHJD:2]. 
buffers per connection at the edges of the network while in connectionless networks buffers at the edge of the network can be shared. As such, the hardware costs of connectionless networks tend to be lower than connection-oriented networks.

In this chapter we present a low-cost ring interconnect as a replacement for buses for small to medium scale real-time multiprocessor systems. This ring will be used in our dual-ring interconnect for our architecture, as described in Chapter 3. Despite that the ring is connectionless we show with an SDF model that throughput guarantees can be given at the application level. Guarantees can be given by reserving "slots" for each master. Reserved but empty slots can be claimed by other masters which makes the network work-conserving. Under the assumption that slaves always accept data in a single cycle, we show that only one FIFO buffer in the NI is needed as this buffer can be shared between streams. The sharing of these buffers enables the support for all-to-all communication using the ring interconnect. Furthermore, there is no need for FIFO buffers in the routers because no contention or head-off-line blocking can occur inside the ring network.

The organization of this chapter is as follows. We first introduce a description of the ring network in Section 4.2. In Section 4.3 we introduce our algorithm which provides fair bandwidth for every NI. A dataflow model for temporal analysis is presented in Section 4.5. Section 4.6 describes the hardware costs of the ring interconnect. We will present the conclusions of this chapter in Section 4.8.

\subsection{Ring Design}

For multiprocessor systems that are designed without precise knowledge of the applications which will run on these systems, it is often desirable that all masters can communicate with all slaves. However, the support of all-to-all communication can result in an expensive communication network if buffers at the edges of the network cannot be shared between different data streams. In contrast, input and output buffers can be shared in our NIs, reducing the required amount of buffering and thereby the hardware costs.

In order to reduce network complexity, we do not have support for back-pressure: whenever a packet enters the ring, it will travel one hop per cycle until it reaches its destination. As such we require that the receiving slave has to accept the packet in the same cycle it is delivered. This guaranteed acceptance also means we can determine the number of hops a packet will travel and therefore how much time is required to reach its destination. The concept of this guaranteed write acceptance is similar to what is required for many multi-layer buses.

Our ring interconnect is write-only: there is no support for remote reads. If remote data is required from a specific peripheral, this peripheral must act like a master and respond to a request by sending data.

The ring is unidirectional which has various advantages over other alternatives. Routing decisions are trivial as packets on the ring cannot be stalled or de- 
flected [18]. When a new packet arrives and the ring is available, a packet is injected into the ring. The decision whether or not the ring is available for a certain packet is further explained in Section 4.3.

Unserialized multiple master to one slave communication usually requires memory port arbitration. As the topology of our ring interconnect provides automatic serialization, no memory port arbitration is required if a dedicated memory port is used such that data can be written at the same speed as the network can produce data.

\subsection{Ring SLOtTING}

To prevent starvation we implement a bandwidth-reservation algorithm. We call our algorithm "ring slotting": we consider the information contents of each NI a "slot". Each clock cycle, the content of all NIs is passed to their neighbors. This way, slots are cycled around the ring. By numbering these slots, we can uniquely identify each one.

By numbering the NIs themselves as well, we introduce the concept of "owned" slots: a slot which has an identifier which matches the NI it currently resides in, is owned by the NI. We now present the first rule of our arbitration:

Rule 4.1 If a slot identifier matches the identifier of the NI it currently resides in, it is "owned" by that NI. Thus NIs can always use their own slot to inject data onto the ring.

Figure 4.1 shows an example of our slot-based arbitration. In this example a write is stalled one cycle until its "own" slot comes up. Data is transmitted to the NI which will take the maximum number of hops, e.g. three hops. The data is in transit the second and third cycle and delivered in the fourth cycle.

It is clear from Rule 4.1 that waiting time, i.e. the time between emitting the data to the input of the NI and the actual injection into the ring, is limited to $\sigma-1$ where $\sigma$ is the number of peripherals on the ring. Similarly, as each $\sigma$ cycles one slot is available to the peripheral, the available bandwidth is exactly $\frac{1}{\sigma}$.

\subsection{Hardware Design}

In our architecture, we have multiple tiles connected to the ring. Processor tiles contain a dual ported memory for data streams which are connected to the ring output. As such, data is written directly into the memory from the output port of the NI.

All the memories are capable of single cycle reads or writes and as such adhere to concept of "guaranteed acceptance" which is required for the ring network. Guaranteed acceptance means that the hardware connected to the output of a ring NI 


\section{NI 2}

\begin{tabular}{|c|}
\hline Slot ID: 0 \\
\hline Destination: \\
\hline
\end{tabular}

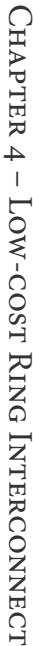

Write: $0 \times 123$ to NI 1
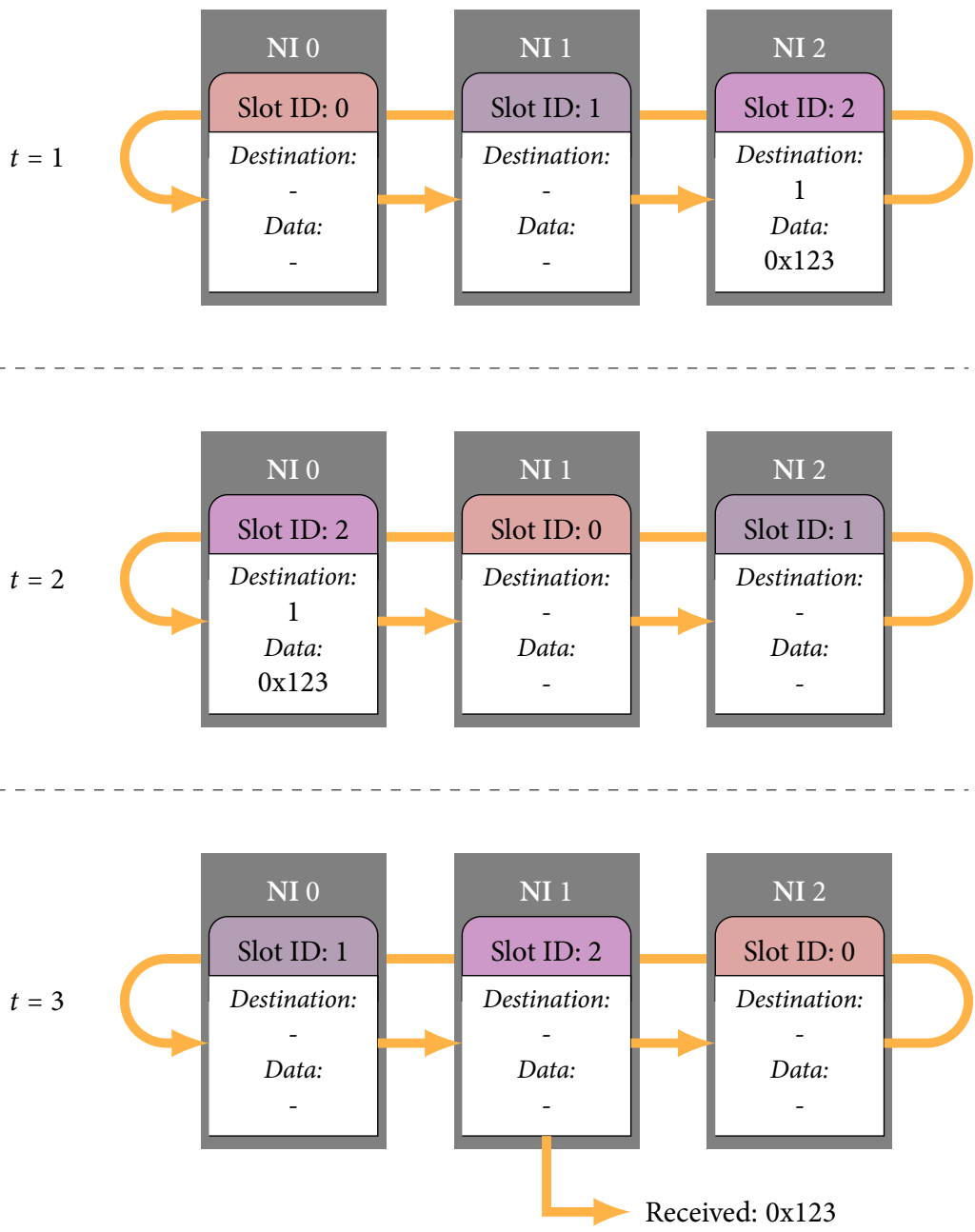

FIGURE 4.1 - Ring slot arbitration example for a word write 
is able to accept data in the same cycle it is presented. We expand the address to not only address data to a specific ring peripheral but also use some bits to denote a specific remote memory location at the target peripheral.

See Figure 4.2 for a schematic overview of a single NI. Our ring network does not have distinct routers; by chaining a number of NIs together the ring topology is formed. The "Arbitration Control" block contains only combinatorial logic.

At the local input port of the network interface there is a small FIFO. This FIFO contains tuples consisting of a network address and a data word. The depth of the FIFO is configurable at design-time. To prevent confusion with the distributed FIFO from Section 3.6.2 we will refer to this hardware FIFO simply as "buffer" for the remainder of this chapter. Whenever it is full, any further writes to the network will stall the CPU. Increasing the buffer depth will allow for larger write bursts without incurring stall cycles. However increasing the buffer depth will also increase the worst-case waiting time as with a full buffer a single data word is dispatched every $\sigma$ cycles. Therefore, with a buffer of depth $\psi$ in the worst case a new word has to wait $\sigma \cdot \psi$ cycles before it is dispatched.

The ring interconnect allows core to core communication and synchronization. We can run applications from the local memories at each processor tile and stream data over the ring interconnect.

\subsection{Temporal SDF Model}

As explained in Section 3.6.2, we can use the write-only scheme from the interconnect for a software FIFO channel where the read and write pointers are placed in

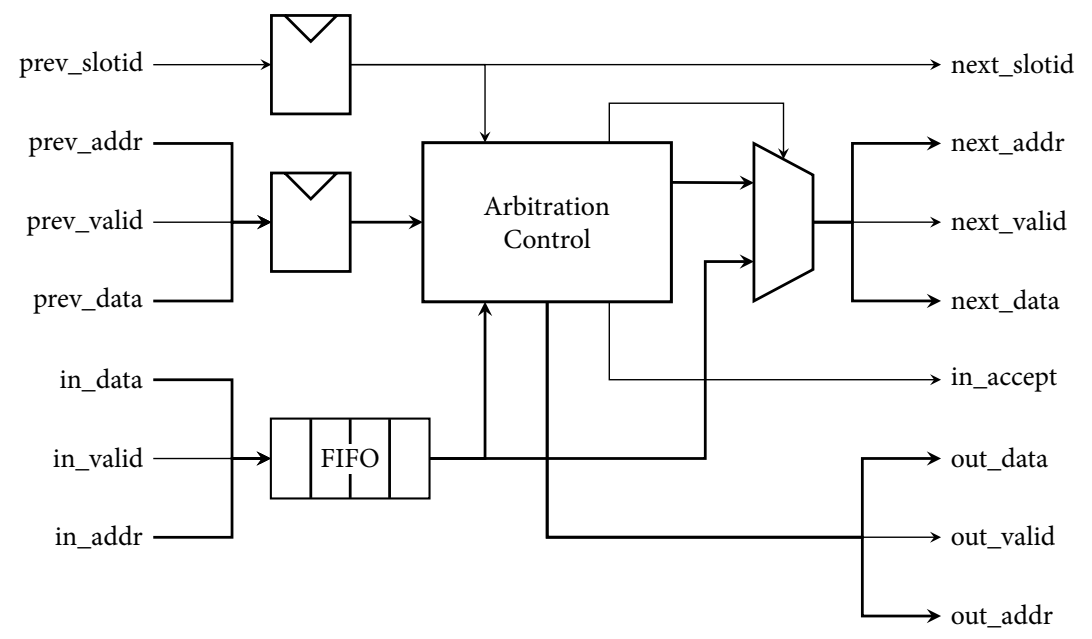

FIGURE 4.2 - Schematic overview of a single NI 


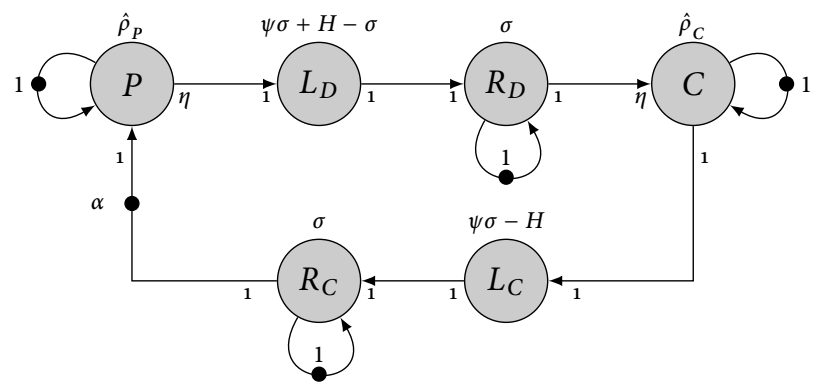

FIGURE 4.3 - SDF graph for a task with core-to-core communication

the memories of both the producer and consumer. This is similar to a credit-based handshake system which can be modeled as an SDF graph [29].

We will now briefly introduce SDF models and the concepts needed to describe the single-ring network in a temporal analysis model. An SDF model is a directed graph $G(V, E)$ consisting of actors $v \in V$ and directed edges $e \in E$. Each edge describes a directed connection between two actors: $e=\left(v_{i}, v_{j}\right)$. The edges represent an unbounded FIFO queue to store tokens.

Actors have a firing rule: when at least a specific number of tokens, called quanta, at the input queue or queues of an actor are available, the actor fires. This means that the required input tokens are consumed instantly and after the execution time of that actor has elapsed a specified number of tokens are instantly produced. When enough input tokens are available, an actor can fire multiple times simultaneously. To prevent parallel execution of a single actor where this is not desired, we can use self-edges with one token to prevent overlapping firings of actors.

We model our software FIFO with capacity $\alpha$ as an SDF graph as is depicted in Figure 4.3. This graph is an abstract model describing both hard- and software where an actor does not need to describe a single hardware or software component. In this model we have a producing actor $v_{P}$ and consuming actor $v_{C}$ with their corresponding execution times: $\rho_{P}$ and $\rho_{C}$. The sharing of a small hardware input buffer, as depicted in Figure 4.2, with capacity $\psi$ by multiple tasks can be modeled by incorporating the waiting time into the execution time. We use the same $\psi$ for all NIs in the rest of this chapter. Each write can cause a wait time $\beta$ of at most $\beta=\psi \cdot \sigma$ cycles if the hardware input buffer is full. Our operating system uses a TDM scheduler with time slice length $\xi$ and as such, a single execution of a task will be preempted $\left\lceil\frac{\rho}{\xi}\right\rceil$ times. In the worst case the input buffer is full each time our task starts or resumes. Therefore, the total maximum wait time $\varphi$ during the write of a complete container is:

$$
\varphi=\beta \cdot\left\lceil\frac{\rho}{\xi}\right\rceil=\psi \cdot \sigma \cdot\left\lceil\frac{\rho}{\xi}\right\rceil
$$

We now incorporate this wait time into the original execution time: $\rho+\varphi=\hat{\rho}$. 
As $\varphi$ directly influences the execution time of the producer and consumer in this model, it should be kept small. This can be done by increasing the length of time slice $\xi$ or decreasing capacity $\psi$. Reducing buffer capacity $\psi$ lowers $\varphi$ but potentially increases the occurrence of processor stall cycles.

Latency from the network is based on the number of hops $H$ that a word travels from the master to the slave with a maximum of $\sigma-1$. The total transit time of a single write is the sum of the waiting time $\beta$ and the number of hops $H$ :

$$
L=\beta+H=\psi \sigma+H
$$

The communication between actors $v_{P}$ and $v_{C}$ is rate limited to $\frac{1}{\sigma}$ which is modeled by actor $v_{R_{D}}$. The number of hops needed to get from $v_{P}$ to $v_{C}$ is $H$. We use two separate actors [84] to model latency and rate between $v_{P}$ and $v_{C}$ where we need to subtract the execution time of the rate limiter from the execution time of the latency actor. The latency from $v_{P}$ to $v_{C}$ is denoted by actor $v_{L_{D}}$ and has execution time:

$$
\rho_{L_{D}}=(\psi \sigma+H)-\sigma
$$

After $\eta$ tokens have been received and consumed by the consumer, a credit token can be sent back to the producer. The communication between $v_{C}$ and $v_{P}$ is again rate limited to $\frac{1}{\sigma}$ which is modeled by actor $v_{R_{C}}$. As the number of hops between $v_{P}$ and $v_{C}$ was $H$ hops, the credit token will travel $\sigma-H$ hops from $v_{C}$ to $v_{P}$ as the total number of hops from $v_{P}$ to $v_{C}$ and back will always be $\sigma$. Together with waiting time $\beta$ we can model the total credit token latency as:

$$
\rho_{L_{C}}=\beta+(\sigma-H)-\sigma=\psi \sigma-H
$$

We will now give the definition of the Cycle Mean (CM) of an HSDF graph [11]. Let $G_{H}$ denote an HSDF graph, $C_{H}$ the set of all directed simple cycles from $G_{H}$ and let $c \in C_{H}$ denote a specific cycle. The weight $w(c)$ of $c$ is defined as the sum of all execution times of the actors on cycle $c$. The number of tokens on cycle $c$ is called $n_{c}$. The mean between the execution times of the actors on the cycle and the number of tokens on the edges of it is the CM:

$$
\lambda(c)=\frac{w(c)}{n_{c}}
$$

The CM of $v_{C}$ gives a weighted average rate between the execution times of all actors in that cycle and the number of tokens on that cycle. This average also describes the throughput of that individual cycle. The Maximum Cycle Mean (MCM) of $G_{H}$ is defined as:

$$
\lambda^{*}=\max _{\forall c \in C_{H}}\{\lambda(c)\}
$$

The throughput of an HSDF graph is the inverse of its MCM. In order to calculate the MCM for the SDF graph from Figure 4.3 we transform it into an HSDF graph [15, 
49, 73]. In the transformation to an HSDF graph, the actors and edges between $v_{P}$ and $v_{C}$ are duplicated to $\eta$ parallel paths with quanta 1 . The self-cycle on $v_{R_{D}}$ becomes a single cycle $c_{1}$ across all copies of $v_{R_{D}}$ with a single token on it:

$$
c_{1}=\left(\left(v_{R_{D_{1}}}, v_{R_{D_{2}}}\right), \ldots,\left(v_{R_{D_{\eta}}}, v_{R_{D_{1}}}\right)\right)
$$

The CM of all cycles from the transformed SDF graph from Figure 4.3 can now be determined. We define the cycle $c_{2}$ as the sending of a complete container from actor $v_{P}$ to $v_{C}$ and the credit from $v_{C}$ to $v_{P}$. This simple cycle is denoted by:

$$
c_{2}=\left(\left(v_{P}, v_{L_{D_{1}}}\right),\left(v_{L_{D_{1}}}, v_{R_{D_{1}}}\right), \ldots,\left(v_{R_{C}}, v_{P}\right)\right)
$$

We denote the CM of cycle $c_{1}$ as $\lambda\left(c_{1}\right)$ :

$$
\lambda\left(c_{1}\right)=\frac{\eta \sigma}{1}=\eta \sigma
$$

Similarly we derive the CM of cycle $c_{2}$ :

$$
\begin{aligned}
\lambda\left(c_{2}\right) & =\frac{\hat{\rho}_{P}+\psi \sigma+H-\sigma+\eta \sigma+\hat{\rho}_{C}+\psi \sigma-H+\sigma}{\alpha} \\
& =\frac{\hat{\rho}_{P}+\hat{\rho}_{C}+2 \psi \sigma+\eta \sigma}{\alpha} \\
& =\frac{\rho_{P}+\varphi+\rho_{C}+\varphi+2 \psi \sigma+\eta \sigma}{\alpha} \\
& =\frac{\rho_{P}+\rho_{C}+2 \psi \sigma\left[\frac{\rho}{\xi}\right]+2 \psi \sigma+\eta \sigma}{\alpha}
\end{aligned}
$$

We can now consider the throughput of the entire HSDF graph by deriving the MCM as is shown in Equation 4.11.

$$
\lambda^{*}=\max _{\forall c \in C_{G}}\left(\lambda\left(c_{1}\right), \lambda\left(c_{2}\right), \hat{\rho}_{P}, \hat{\rho}_{C}, \sigma\right)
$$

From Equation 4.11 we can see that as long as $\lambda\left(c_{2}\right)$ is the largest component in $\lambda^{*}$, increasing the FIFO capacity $\alpha$ directly lowers $\lambda\left(c_{2}\right)$ and thus the MCM. We can also see that when $\alpha$ is sufficiently large, it is no longer the largest component in $\lambda^{*}$ and as such no longer influences the MCM. At this point we can say that the network latency no longer influences application throughput as the execution time of actors $v_{P}$ or $v_{C}$ or the rate limiters $v_{R_{D}}$ and $v_{R_{C}}$ will limit throughput.

Note that increasing the capacity $\alpha$ of the FIFO placed at $v_{C}$ increases the initial number of tokens on the edge between actor $v_{R_{C}}$ and $v_{P}$ but it does not influence the execution time of any of the actors. This means that the latency resulting from the ring interconnect and the FIFO algorithm does not change by altering the capacity of the FIFO instance.

For most stream processing applications latency requirements are usually less tight than throughput and as such using buffers with sufficient capacity can often be used to compensate for latency. 


\subsubsection{WORK-CONSERVING}

The use of unique slots on the ring interconnect provides strict latency and bandwidth guarantees required for real-time applications. However just like most GT networks it is not work-conserving as unused slots remain empty which we will now change with a small modification to our arbitration policy.

Since we know when a slot reaches the rightful owner, who may or may not use the slot, we can use an empty slot to address peripherals up to and including the owner of the slot. This would allow the use of otherwise wasted bandwidth without interfering with the GT packets and thus without interfering with all guarantees. We now define the second rule in our arbitration policy:

Rule 4.2 If a NI is ready to send data, the current slot is empty and the owner of the slot is not reached before the destination NI is reached, data can be injected into that slot.

This small addition adds a small amount of hardware to the arbitration and injection logic but makes the ring interconnect work-conserving. By making more slots available we increase the upper bounds of the bandwidth based on the distance a packet has to travel on the ring.

We define the GT bandwidth as $B=\frac{1}{\sigma}$. We use $M_{\text {hops }}$ to denote the number of hops a packet has to travel between peripherals. As we are not allowed to use any of the slots belonging to NIs between the source and destination peripheral, we can potentially use $\sigma-M_{\text {hops }}$ slots out of $\sigma$ slots. We can now derive the upper bound on the available bandwidth, called $\hat{B}$ for traffic between two NIs:

$$
\hat{B}=B+\frac{\sigma-M_{\text {hops }}}{\sigma}=\frac{\sigma-M_{\text {hops }}+1}{\sigma}
$$

We can see from Equation 4.12 that when the distance data has to travel on the ring increases, the upper bandwidth bound decreases proportionally.

\subsubsection{External Memory}

As long as the stream processing can exploit data locality while containers are relatively small the use of local memories suffices. When this is not the case, larger memory buffers might be required. An example of this is a video deinterlacer which requires the buffering of at least half a frame. For pixels using 32-bit color at VGA resolution $-640 \times 480$ - this video data already requires more than $600 \mathrm{kB}$ of memory.

For these types of buffers, we can use the shared memory which is shared between all processor tiles using a latency bounded tree shaped interconnect, as described 


\begin{tabular}{rccccccc}
\hline & \multicolumn{2}{c}{ Complete Design } & \multicolumn{2}{c}{ Ring } & \multicolumn{2}{c}{ Ratio } \\
CPUs: & Sl.Regs: & LUTs: & Sl.Regs: & LUTs: & Sl.Regs: & LUTs: \\
\hline 2 & 5746 & 7905 & 115 & 119 & $2.0 \%$ & $1.5 \%$ \\
4 & 11492 & 15810 & 237 & 241 & $2.1 \%$ & $1.5 \%$ \\
8 & 22984 & 31620 & 481 & 452 & $2.1 \%$ & $1.4 \%$ \\
16 & 45968 & 63240 & 977 & 1006 & $2.1 \%$ & $1.6 \%$ \\
32 & 91936 & 126480 & 1985 & 1975 & $2.2 \%$ & $1.6 \%$ \\
\hline
\end{tabular}

TABLE 4.1 - Logic usage on a Virtex-6 FPGA showing a linear scale in resource usage for our ring interconnect.

in Section 3.7. The use of instruction and data caches for each CPU lowers the contention for access to the external memory and improves the average case throughput and access latency for these large buffers. We have used this memory for the video deinterlacer task - which stores output pixel data from our video decoder application - and the frame buffer for the DVI controller, as explained in Section 7.1.1.

In theory this shared memory can be used to store data for the C-FIFO channels which enables the use of large data containers or a large number of data containers for a FIFO channel at the cost of high latency access to data. Additionally, it is difficult to give a worse case bound on the access latency and throughput due to the unpredictability of the external memory caused by other traffic using the shared memory. However, experiments with our test application showed that this communication method can reduce throughput by a factor 2 , as discussed in more detail in Section 7.1.1 and as such this approach is not very desirable.

\subsection{Hardware Costs}

We implemented our ring interconnect for an MPSoC with 16 CPU cores on a Xilinx ML-605 prototyping board.

See Table 4.1 for more details on hardware usage. These results indicate that the hardware usage of our ring scales linearly with the number of CPUs. The minor relative increase in hardware costs is due to the increasing width of the address bus for addresses on the ring itself when the number of NIs increase. We can see that the ring interconnect accounts for only $\sim 2 \%$ of the total hardware costs which is small compared to other components in the design.

\subsubsection{Synthesis Results and Power Estimates for ASICs}

As stated before, the structure of the ring results in low hardware costs which can be seen from the presented hardware resource costs for a Virtex-6 FPGA. We also synthesized our ring interconnect into ASIC logic using a standard cell implementation, where low hardware costs are also observed. The Synopsys synthesis tooling 


\begin{tabular}{ccccc}
\hline $\begin{array}{c}\text { Voltage } \\
V\end{array}$ & $\begin{array}{c}\text { Area } \\
\mu m^{2}\end{array}$ & $\begin{array}{c}\text { Speed } \\
G H z\end{array}$ & $\begin{array}{c}3 \% \text { Load } \\
m W\end{array}$ & $\begin{array}{c}100 \% \text { Load } \\
m W\end{array}$ \\
\hline $1.0 \mathrm{GP}^{2}$ & 1100 & 2.0 & $1.24(5 \%)$ & $1.60(6 \%)$ \\
$1.2 \mathrm{LP}^{3}$ & 1107 & 1.0 & $0.79(0.09 \%)$ & $1.00(0.07 \%)$ \\
\hline
\end{tabular}

TABLE 4.2 - Post-synthesis results of $65 \mathrm{~nm} \mathrm{ST} \mathrm{implementation} \mathrm{in} \mathrm{ASIC} \mathrm{logic,} \mathrm{leakage} \mathrm{is}$ specified after power.

was used with the low power and low leakage $65 \mathrm{~nm}$ library from STmicroelectronics.

Synthesis results indicate that for every NI $1100 \mu \mathrm{m}^{2}$ of silicon is required, see Table 4.2. While wiring and the small input buffer are not included, we can compare this rough estimate to specified area of routers of other interconnects. Compared to the latest version of Æthereal light, our $\mathrm{NI}^{1}$ is 20 times smaller than their smallest GS router $\left(0.07 \mathrm{~mm}^{2}\right)$ [25].

\subsection{Design IMPROVEMENTS}

The presented design has a low hardware cost and is very suitable for real-time stream processing applications in a homogeneous multi-core architecture. However, we recognize that in this design aspects of the single ring can be improved depending on the use case. In this section we present potential improvements.

\subsubsection{Reorder Slots to Improve Average Latency}

Using ordered slots on the ring is a design decision which is not imposed by our arbitration policy but rather a result of a straight-forward choice. For example, in the current design the number of available slots for traffic to a specific tile arrive in numerical succession. As a result, our arbitration policy will disallow the use of a successive series of slots depending on which NI they belong to and which NI is the destination of the current write. This results in a sort of sliding window of available slots - assuming all arriving slots are empty - to use in such a transmission for every revolution of the ring. In practice, due to the use of ADCs in radio receiver applications will see transmission of data tokens at an interval instead of transferring a continuous burst of data. As such, it might be better for the average case to shuffle the order of the slots on the ring to facilitate the availability of multiple smaller but more evenly spaced windows for such transmissions.

Note that our arbitration policy operates per slot. Furthermore, no knowledge of which NIs are used by streams nor which streams overlap is used - as was the case

\footnotetext{
${ }^{1}$ Including all logic and registers, without the optional small input buffer

${ }^{3}$ General Purpose Logic, Low Voltage: $1.00 \mathrm{~V}, 25^{\circ} \mathrm{C}$

${ }^{3}$ Low Power Logic, Nominal Voltage: $1.20 \mathrm{~V}, 25^{\circ} \mathrm{C}$
} 
in this chapter. As such, reordering the slots has no impact on the arbitration policy or the guarantees we derive from it. Therefore, the same number of slots will be considered to be available by our arbitration policy per revolution of the ring. Thus upper and lower bounds on throughput and latency will be preserved.

\subsubsection{Reorder Slots to Improve Power Efficiency}

Using gray coding has a similar effect as reordering the slots but aside from more evenly distribute the available slots it can reduce the amount of power used to transfer the slot ID between NIs. In this approach the numbering of the IDs of the NIs is not altered. Because we only reorder the sequence of slots, the same number of slots will be considered to be available by our arbitration policy per revolution of the ring and the lower and upper bounds on throughput and latency are preserved.

If the IDs of the NIs would be changed to be non-linear in sequence, the workconserving aspect of our arbitration policy would no longer function. As workconserving behavior is enabled by applying Rule 4.2 , logic in the ring NI is required to be able to determine which NIs are passed by claiming an unused slot. As such, the logic implementing Rule 4.2 would either become much more complex or require some form of lookup table.

\subsubsection{Clock Gating}

While slot identifiers have to be cycled around the ring, the contents of the address and data registers within a slot are only useful when they are loaded with valid data. It should be possible to clock gate the address and data registers within all NIs to only enable them when valid data is to be stored. In this fashion, address and data registers will be idle until needed.

\subsubsection{SLOT MASKING}

In our model we assume every NI can use any free slot which does not interfere with the arbitration policy. However, for most applications, the communication channels between tasks are known at compile time. This knowledge can be used to determine how many streams pass a specific NI. By masking which slots are available for a specific NI we can effectively allocate this bandwidth to other (earlier on the ring) NIs, thereby increasing the guaranteed minimum throughput for that NI. At the same time, as multiple slots will now be available to a specific NI every rotation, the worst-case latency can be reduced.

While this approach does not allow bandwidth allocation per task - but rather per tile - it can improve the worst-case throughput by applying this application knowledge. We implemented slot masking in an experimental build but did not explore this option further and omitted it in the design instances used in our case studies in order to minimize the hardware costs of the single ring. As such, this technique is not further used in this thesis. 


\subsubsection{Slot Reallocation}

Unused PEs on the ring could be forced to yield their slot to be available to other NIs on the ring. This could be implemented with small changes to the arbitration policy and can improve for example the bandwidth for communications traveling many hops.

A similar improvement is to allocate multiple slots per NI per rotation of the ring. This is a simple method to improve the worst-case bandwidth for a specific NI but can impact the guarantees that can be derived for other NIs.

\subsubsection{MAPPING}

Currently, allocation of tasks to PEs can be done by hand or by Omphale. The mapping performed by Omphale is based on an algorithm which attempts to distribute the loads of tasks over cores evenly.

As such, we currently are missing a mapping algorithm which takes the properties of the ring interconnect into account. Based on the distance - measured in number of hops traffic over the ring will take - between cores, the worst-case latency and bandwidth should be computed during mapping to determine if a mapping of an application would result in acceptable real-time performance.

A more advanced version of such an algorithm can also take into account which data streams will occupy the same links between NIs and the bandwidth and latency requirements for each overlapping stream. When masking of slots is used, see Section 4.7.4, suitable slot masks could be computed to distribute bandwidth more appropriately based on a specific mapping.

\subsection{Conclusion}

In this chapter we presented a low-cost communication ring for real-time multicore stream processing architectures as used in the SDR domain. Our single-ring interconnect realizes low hardware cost by sharing buffers within a NI. This is possible because the ring provides automatic serialization and requires guaranteed acceptance at the slaves. Despite being connectionless, our ring provides bandwidth and latency guarantees by reserving slots for each master. The work-conserving scheduling policy of the ring interconnect allows tasks to use the slack of other tasks which can improve the average throughput at the application level.

We derived an SDF model for the communication channel between a master and a slave which can be used to determine the required capacity in order to reduce the effects of network latency on application throughput.

We implemented our design of the ring interconnect in an MPSoC on an FPGA and in ASIC logic. On the FPGA we found that our interconnect scales linearly and occupies $2 \%$ of the resources used in the design. 


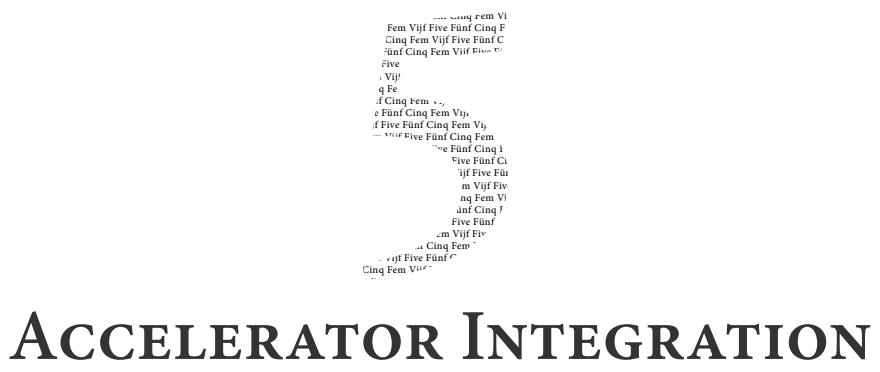

ABstract - Connection-oriented GT mesh-based NoCs have been proposed as a replacement for buses in real-time stream processing systems but are currently rarely used as hardware cost tends to be higher than conventional interconnects. An interconnect with a ring topology was introduced in Chapter 4 as a low-cost alternative for use in medium scale homogeneous MPSoC designs. Cost-effective integration of stream processing accelerators requires an extension of this single-ring interconnect.

In this chapter we present a dual-ring communication infrastructure for heterogeneous MPSoC designs. Data and credits are transferred between tiles using their separate oppositely-directed rings. The minimum throughput is determined by analysis of a CSDF model for a system with communication between accelerators and processors.

\subsection{INTRODUCTION}

Programming of homogeneous MPSoCs tends to be easier than the programming of heterogeneous MPSoCs. However, the use of general purpose processors for DSP applications is inherently less efficient compared to the use of dedicated hardware. As such, the use of stream processing accelerators which contain dedicated logic can improve throughput. However, an interconnect which supports stream processing accelerators should not be so complex that it negates the energy and performance advantages gained from using accelerators. At the same time, such an interconnect should provide real-time guarantees as required by many stream processing applications, for example in the SDR domain.

In order to improve the efficiency and performance of our system, we propose to use stream processing accelerators. Integrating stream processing accelerators in a

Large parts of this chapter have been published in [BHJD:4]. 
memory-mapped MPSoC introduces new issues as these accelerators usually work on a stream of data and as such have no notion of addresses. Communication with these accelerators requires an interconnect which supports hardware flow control. We will extend the single-ring interconnect from Chapter 4 with support to facilitate the integration of stream processing accelerators.

Our contributions in this chapter are as follows. We introduce a dual-ring communication infrastructure for heterogeneous MPSoCs with accelerators intended for stream processing applications. Hardware flow control is credit-based where the number of credits denote the depth of the buffer between a producer and a consumer. A producer consumes credits upon producing data and a consumer sends the producer a credit whenever it consumed data. As flow control is implemented in hardware by means of a small shell in each NI of the dual-ring interconnect, the number of simultaneous FIFO channels for this implementation is limited. As such, all communication involving stream processing accelerators is handled in hardware, including streaming data from processor-to-accelerator or vice versa, while processor-to-processor communication is handled in software by our C-FIFO algorithm, which was explained in Section 3.6.2 and modeled as an SDF graph in Section 4.5. We present a CSDF [7] model for hardware-based flow control in order to derive application-level throughput guarantees. This model is valid for processor-to-accelerator communication when said processor only executes a single task. We show by means of a real-time Phase Alternating Line (PAL) video decoder application, which is described in Section 7.1.3, that adding the second ring and accelerator results in a significant improvement in throughput but results only in a relatively small increase of the hardware cost.

The outline of this chapter is follows. Section 5.3 presents the dataflow model of the hardware flow control and in Section 5.4 hardware costs are presented. The conclusions are presented in Section 5.5.

\subsection{SINGLE-RING INTERCONNECT}

Our dual-ring interconnect combines two unidirectional rings to support software and hardware-based flow control. By only applying hardware flow control for data streams involving accelerators, we keep hardware costs low while the use of software flow control between software tasks maximizes flexibility in communication patterns.

We will now briefly summarize the properties of our single-ring interconnect as was explained in Chapter 4. Our packet-switched unidirectional interconnect for all-to-all communication has low hardware cost. Our slot allocation algorithm for the ring, as explained in Section 4.3, provides GT traffic and makes the ring workconserving. All-to-all communication is supported without using further internal buffers, aside from registers between NIs. This results in low hardware cost and does not reduce predictability. 
A ring interconnect consists of a number of NIs that are chained together from one to another, forming a ring. When a packet is received from a neighboring NI, it is either ejected when the current NI is its destination, or passed on to the next NI if it is not. Packets that are to be injected, enter the ring when it is available: which means it is injected when the current NI has no packet to pass along and the arbitration policy allows injection. Because packets have a single route between two tiles, it is guaranteed that data writes arrive in the same order as they were issued. As routing is trivial, we consider the "router" to be part of the NI.

To keep hardware costs low, our single-ring interconnect relies on the concept of guaranteed acceptance: connected tiles are required to accept and register any packet in a single cycle at any time, which is similar to many multi-layer buses. As such, data is ejected from the ring whenever it reaches its destination. This means that there is no need for back-pressure or packet deflection to manage packet flow within the ring. This also allows us to determine the transit latency precisely since we know for every packet how many hops it will need to reach its destination.

The nature of a ring provides serialization for all NIs, preventing the need for memory port arbitration. As input and output buffers can be shared between streams in our NIs, there is no limit to the number of simultaneous streams that can be multiplexed over the same NI.

\subsubsection{Credit Ring}

We extend the interconnect consisting of a single, unidirectional data ring as described in the previous section with a second ring for flow control for stream processing accelerators. To keep hardware costs low, it is undesirable to have large buffers at the inputs of accelerators to hold large tokens when processing can be fine grained. This means containers for the accelerator input FIFO could be as small as a single data word and buffers have to hold at least one such container. To prevent a large communication overhead on the data ring, we will use this second ring to send credits between consumers and producers.

By using a credit-based handshake [47], we implement flow control for accelerators. In this flow control scheme, we have a producer-consumer pair with a number of credits. The number of credits available at the producer determines how many data containers can be buffered at the consumer. When the producer wants to send data, it is required to have at least a single credit available. When no credits are available anymore, the producer has to wait for the consumer to free up its buffer. When a consumer consumed a data container, it sends a credit back to the producer to signal that it freed up space for a single data container.

In this case, the number of credits indicate the number of data containers that can be buffered at the input of an accelerator. As long as a producing accelerator has credits and input data, it can send data to another accelerator.

As credits are not unique, we only need to transfer the fact that a credit is present; this can be done in a single bit instead of 32 bits for a data word. As such, the credit 


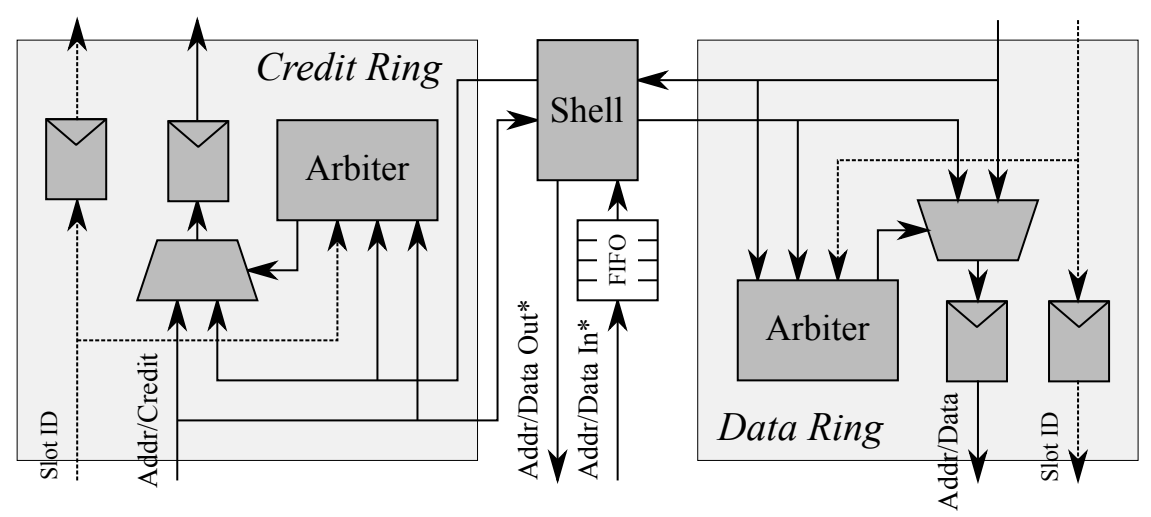

FIgURE 5.1 - Schematic overview of a single NI. *Accelerators only read and write data and omit addresses.

ring requires less hardware than the data ring.

The credit ring has the same limitations as the data ring: high throughput and low latency is only possible when processing is kept local, i.e., data is sent to a neighbor which is only a few hops away. To keep latency low and prevent the needless intersection of credit streams, the credit ring transfers its payload in the opposite direction of the data ring. This means that if data is transferred $H$ hops, a credit will traverse $H$ hops back from the data consumer to the data producer.

\subsubsection{RING SHELL}

Credit-based flow control is implemented in the NI in a small shell which makes use of both rings. This shell passes traffic for the software FIFO to the data ring. Data traffic to or from accelerators is subject to hardware flow control.

Figure 5.1 shows a schematic overview of a NI of the dual-ring network, consisting of the routers of both the data and credit ring and the shell. At the local input port of the NI there is a small FIFO which has a capacity of one word for accelerators and has a configurable number of locations for processor tiles. This FIFO holds tuples consisting of a network address and a data word. Addresses are provided when writing data by processor tiles while accelerators only write data. The depth $\psi$ of the FIFO is configurable at design-time. To prevent confusion we will refer to this hardware FIFO simply as "buffer" for the remainder of this chapter. Whenever this buffer is full, any further writes from the local tile to the network will stall this tile.

Each shell contains a single credit-return address per input and single dataforwarding address per output. As such, our shell uses static or key-hole addressing which prevents the need for dynamic address generation. As data arrives at the accelerator tile, the shell will pass it to the inputs of the accelerator. When the accel- 
erator accepts the data at one of its inputs, a credit will be sent to the return address. Every input for an accelerator is buffered by the shell where at least a single input token is buffered. Data produced by an accelerator is passed to the shell which will forward data to the data-forwarding address as soon as credits are available. In this fashion, data is streamed from one accelerator to the next.

While flow control supports the use of multiple credits per stream, input buffers with a matching capacity at the accelerators are required. In the current implementation we use a fixed depth of at least two credits per stream. This enables concurrent processing for accelerators using hardware flow control. Often, only a low number of credits already results in the required minimum throughput for the applications considered in our case studies.

\subsection{Dataflow Model}

The dual-ring interconnect is used for software FIFO communication between processor tiles and hardware FIFO communication for all other combinations between tiles. The software FIFO communication has been modeled as an SDF graph, as was shown in Chapter 4.

An SDF model is a directed graph $G=(V, E)$ with actors $v \in V$ and directed edges $e \in E$. Each edge $e_{i, j}$ describes an unbounded queue for atomic data objects called tokens between actors $v_{i}$ and $v_{j}$. The head and tail of each edge is annotated by quanta which denote the number of tokens an actor will consume or produce. Every actor has a firing duration $\rho_{v}$. An actor can fire when its incoming edges have at least the number of tokens as denoted by their quanta. To prevent concurrent firing where this is not desired, self-edges are used with a single token to prevent overlapping firings of actors.

CSDF [7] extends SDF by introducing the concept of phases. Furthermore, each actor has a cyclic behavior during which its phases are fired. The firing duration for every phase $p$ is denoted as $\rho_{v}[p]$. Both firing durations and quanta are expressed as a list of values with a length equal to the number of phases of the corresponding actor.

The original CSDF definition from [7] specifies that an implicit self-edge is present on every actor. However, in this chapter we define that actors in a CSDF graph do not have an implicit self-edge and as such phases could fire concurrently. To prevent token reordering all phases of an actor that does not have a self-edge with one token must have the same firing duration. If parallel execution of phases of the same actor is not desired, a self-edge is added with a single token to the CSDF actor.

In order to keep hardware costs low, inputs of an accelerator tile should have a FIFO with a very small capacity as we assume that most stream processing accelerators can work on individual data words. Processor tiles on the other hand, transfer 


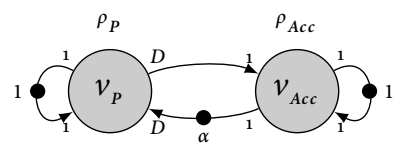

FIGURE 5.2 - Potentially dead-locked model for accelerator communication

larger chunks of data to minimize overhead resulting from the acquire and release calls on containers in the software FIFO administration.

If we would attempt to capture the exchange of data containers between a processor tile and an accelerator tile in a simple SDF model containing a Producer-Consumer pair of actors, we see that this model is not accurate because it models that all $D$ words are produced at once when the task $v_{P}$ finishes its execution instead of word by word during the execution of the task. The result is shown in Figure 5.2 and contains actors $v_{P}$ and $v_{A c c}$ where $v_{P}$ produces data in FIFO containers of size $D$ words and $v_{\text {Acc }}$ consumes data one word at a time. The depth of the FIFO between both actors is expressed in data words and denoted by $\alpha$. When the hardware FIFOs should be kept small for cost reasons, it is desirable to make $\alpha$ smaller than $D$. However in this case the model will deadlock.

We will now introduce a new model for temporal analysis of the hardware FIFO communication. To this end we derive a CSDF model which can be used to determine whether the requirements are satisfied for a specific real-time stream processing application. We need CSDF as we just showed that SDF is not sufficiently expressive to accurately model the communication between a processor and an accelerator.

In our CSDF model, the incremental transference of data $v_{P}$ to $v_{A c c}$ is modeled by the explicit release of individual data tokens where every token denotes a data word. In Figure 5.3, actor $v_{P}$ produces $D$ times a token (notation $\langle D \times 1\rangle$ ), each of which is released explicitly after each phase. Note that the sum of all firing durations for each phase of $v_{P}$ from Figure 5.3 is identical to the firing duration of $v_{P}$ from Figure 5.2 and for simplicity we can assign the first phase the summed duration. At the start of the firing of $v_{A c c}$, one token will be consumed and at the completion one credit token is emitted.

Note that if $\alpha=1$, parallel execution is impossible as $v_{P}$ and $v_{A c c}$ will execute one after another. As such, we assume that $\alpha \geq 2$ where increasing FIFO capacity im-

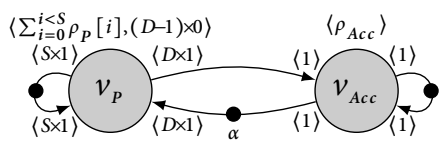

FIGURE 5.3 - CSDF model of hardware FIFO communication 
proves pipelining and throughput. We will show that a minimum FIFO buffer capacity can be computed given a minimum throughput requirement.

In our system a processor will stall if a write can not complete. During a stall the processor does not execute instructions. A write can not complete if the buffer in the network interface is full. How often this buffer is full during a write depends on the actual moments in time that $v_{A c c}$ consumes its data.

Firing durations must be known before analysis of the latest possible consumption moments can be performed. However, to obtain the firing duration an iterative analysis approach would be required, as is described in [32]. In such an iterative approach the firing durations are updated based on schedules computed in the previous iteration of the model. This iterative approach complicates analysis and drawing conclusions about bottlenecks. Therefore, we use an analysis method flow without iterations which implies that all firing durations must be known before schedules are computed. As such, the actual consumption moments are not known at design-time and therefore the total time that the processor will stall is unknown.

As a consequence it is not possible to determine at design-time how many cycles are left for other tasks executing on the same processor. Therefore, to obtain a compositional analysis method we do not allow sharing of a processor by multiple tasks if one of these tasks communicates with an accelerator.

At the completion of every phase of $v_{P}$ a write is issued which may stall the processor. However, as writes only occur at the end of a phase and not during its firing, the processor will never stall during the firing of a phase. As such the firing durations of all phases of $v_{P}$ are not affected by the stalling of the processor.

When we consider a FIFO channel between accelerators, a producing accelerator would write output word by word to the consuming accelerator which will send a credit for every accepted word. As such, the model from Figure 5.3 can also be applied to accelerator-to-accelerator communication when $D=1$.

One type of data exchange between tiles that has not been covered so far, is streaming data from an accelerator to a processor tile. As shown in Figure 3.2 on page 35, data arrives at a processor tile in a hardware FIFO which is connected to the shell from the NI like an accelerator. Data delivered to the hardware FIFO is directly available to a task running on the processor. As such, from a modeling perspective, this case is no different than inter-accelerator communication.

\subsubsection{Channel Description}

The model from Figure 5.3 only describes the communication between a producer $v_{P}$ and consumer $v_{A c c}$ where the interaction at the end points are described. As such the interconnect that actually transports the data is assumed to be ideal: it introduces no latency and has unlimited bandwidth. In an actual system, data is transferred by an interconnect which introduces latency and imposes a bandwidth limit. 
These properties can be described by a Latency-Rate Server model [84] which is shown in Figure 5.4.

By adding two actors in both the data and credit path, we can express latency and rate for both channels. The latency actor $v_{L}$ from Figure 5.4 introduces a delay expressing the worst-case latency experienced by any packet that traverses the interconnect to an arbitrary tile. This includes all wait times like access delays and queuing effects and thus requires a deterministic interconnect which can provide such guarantees. Because there is no queuing in the network itself, the latency for a packet going from $v_{P}$ to $v_{C}$ in Figure 5.4 is $\rho_{L}+\rho_{R}$. Therefor, the firing duration of $\rho_{L}$ should be the worst-case latency reduced by the firing duration of $\rho_{R}$.

The second actor $v_{R}$ is used to model the maximum rate or bandwidth of the interconnect. In the ideal case, both the firing duration $\rho_{R}$ and latency $\rho_{L}$ are 0 which implies unlimited bandwidth and no packet latency. As this is never the case, the self-loop on $v_{R}$ determines the rate at which tokens are passed on to $v_{C}$. As $v_{R}$ can fire once every $\rho_{R}$ time units, its maximum throughput will be $\frac{1}{\rho_{R}}$.

We will now use the properties from the ring interconnect as described in Section 5.2 to obtain the CSDF model for hardware FIFO communication on the dualring interconnect. As both credits and data traverse the interconnect, both paths in the model use a Latency-Rate Server to describe the channel as is shown in Figure 5.5.

From the ring-slotting arbitration as described in Section 4.3 we see that a ring with $N$ tiles will fairly distribute all available bandwidth under full load and as such the worst-case throughput will be $\frac{1}{N}$. The worst-case latency for the transfer of a data packet over the ring is the combination of the stall time before a packet is admitted onto the ring and the traversal time on the ring itself. The worst-case stall time occurs when the input buffer at the NI is filled with $\psi$ data words and we missed the owned slot of the NI by one cycle. After $N-1$ cycles, the first data packet is injected into the owned slot from the buffer and every $N$ cycles after that, the next data word is injected into the owned slot on the ring until all data is transmitted. As such the stall time is $\beta=(N-1)+(\psi-1) N=\psi N-1$. The worst-case latency for a single packet is as such the sum of stall time $\beta$ and the traversal time based on the number of hops $H$ a packet takes until it reaches its destination: $\beta+H=\psi N-1+H$. As credit tokens traverse the dual-ring in the opposite direction of the data, the latency for both data and credit packets is identical.

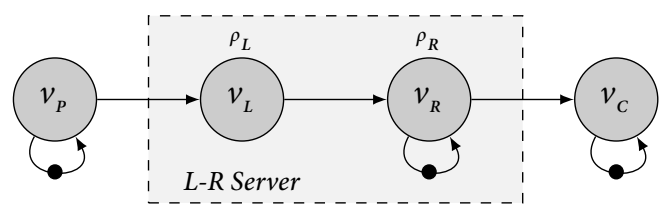

FIGURE 5.4 - HSDF model of a Latency-Rate Server 


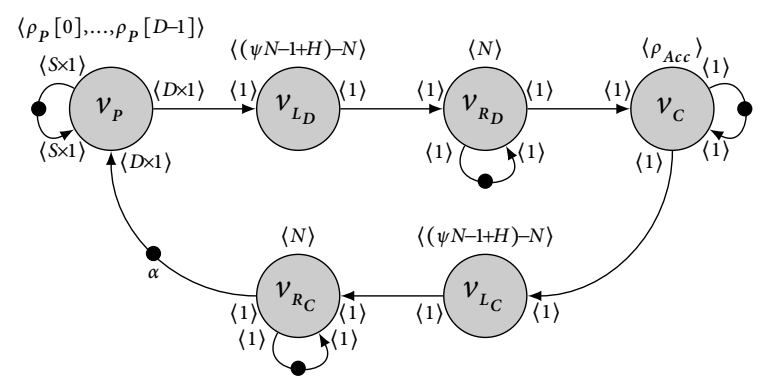

FIGURE 5.5 - CSDF model of hardware FIFO including channel characteristics

\subsubsection{Guaranteed Throughrut}

In order to determine exact throughput and latency bounds, we need to transform the CSDF model from Figure 5.5 to its HSDF counterpart. HSDF is a simplified form of SDF where all quanta are one.

The model from Figure 5.5 does not contain the stall time introduced by the network at the producer when the input buffer at the NI is full. When we are only interested in the worst-case stall time before a packet is admitted into the input buffer at the NI, we see that within $N-1$ cycles a packet from the input buffer is injected into the ring. One cycle later the input buffer can accept a new packet in the input buffer. As such, in the worst-case, at the end of each phase of $v_{P}$, writing a single data word stalls the processor $N$ cycles.

This stall time can be aggregated as the total stall time for any actor $v$ during the write of $D$ words:

$$
\varphi=N \cdot D
$$

This wait time can be incorporated into the original firing duration of actor $v: \hat{\rho}=$ $\rho+\varphi$. As $\varphi$ directly influences the firing duration of $v_{p}$, it should be kept small. This can be achieved by reducing the capacity of the input buffer $\psi$ at the NI. However, lowering $\psi$ can make the average case throughput worse by increasing the amount of stall cycles.

We need to transform the model from Figure 5.5 from CSDF to its HSDF counterpart [7] in order to determine its MCM. The throughput is the inverse of the MCM. The MCM is the maximum of all Cycle Means (CMs) of every simple directed cycle $C$ in the HSDF graph $G$ :

$$
\lambda^{*}=\max _{\forall C \in G}\{\lambda(C)\}
$$

Where the $\mathrm{CM}$ of a cycle $C$ is defined as:

$$
\lambda(C)=\frac{w(C)}{\tau}
$$




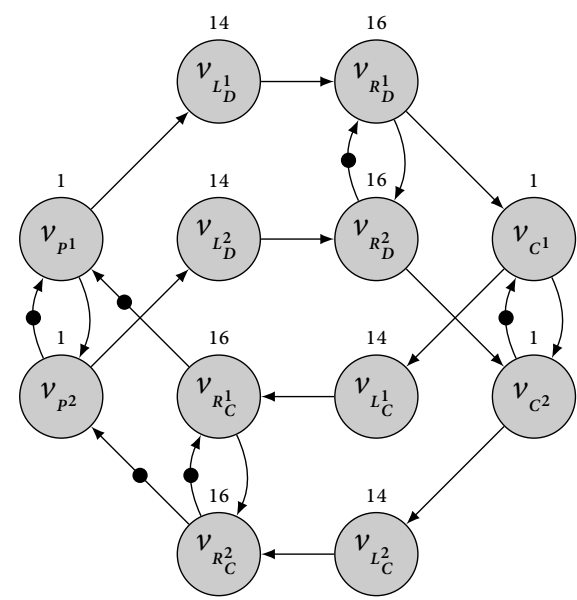

FIGURE 5.6 - HSDF obtained from transformation of the CSDF model from Figure 5.5

Here $w(C)$ is the sum of all firing durations of all actors on $C$ and $\tau$ is the sum of number of tokens on all edges in $C$. The cycle with the largest CM is called the critical cycle.

In the transformation from CSDF to HSDF of the model for hardware FIFO communication, the value of $\alpha$ and $D$ determine the number of actors in the resulting model and the critical cycle through them. We will show the transformation for the case where $\alpha=2$ and $D=2$ and derive the MCM. As the resulting HSDF model will have many cycles, rather than obtaining the MCM symbolically, we will present a numerical example. We use the following parameters: input buffer $\psi=1$, worstcase traversal time is $H=15$ for traffic on a ring with $N=16$ tiles. Additionally, we define that $\forall i \bullet \rho_{P}[i]=\rho_{A c c}=1$, where $i$ denotes all phases for $v_{P}$.

We transform the CSDF model to a HSDF model [7], which is shown in Figure 5.6. Using the parameters as specified before, we can now numerically calculate the firing duration for each actor. We find that two cycles have the maximum CM and as such are critical:

$$
\begin{aligned}
& c_{1}=\left(v_{P^{1}}, v_{L_{D}^{1}}, v_{R_{D}^{1}}, v_{C^{1}}, v_{L_{C}^{1}}, v_{R_{C}^{1}}, v_{P^{1}}\right) \\
& c_{2}=\left(v_{P^{2}}, v_{L_{D}^{2}}, v_{R_{D}^{2}}, v_{C^{2}}, v_{L_{C}^{2}}, v_{R_{C}^{2}}, v_{P^{2}}\right)
\end{aligned}
$$

The MCM can now be determined:

$$
\lambda^{*}=\lambda\left(c_{1}\right)=\lambda\left(c_{2}\right)=\frac{62}{1}
$$

The transformation of the CSDF model for other values of $D$ and $\alpha$ is done in a similar fashion. For completeness, we show the MCM for various combinations of 


\begin{tabular}{ccccccc}
\hline & \multicolumn{6}{c}{ Container size in number of words $(D)$} \\
& 1 & 2 & 3 & 4 & 5 \\
\hline & 1 & 62 & 124 & 186 & 248 & 310 \\
& 2 & 31 & 62 & 93 & 124 & 155 \\
FIFO capacity $(\alpha)$ & 3 & $\frac{62}{3}$ & $\frac{124}{3}$ & 62 & $\frac{248}{3}$ & $\frac{310}{3}$ \\
& 4 & 16 & 32 & 48 & 64 & 80 \\
& 5 & 16 & 32 & 48 & 64 & 80
\end{tabular}

TABLE 5.1 - MCM for various $D$ and $\alpha$ on for hardware FIFO communication between a processor and accelerator.

$D$ and $\alpha$. The resulting MCMs are presented in Table 5.1 where we can see that the dual-ring interconnect is rate limited in this example by $v_{R_{D}}$ when $\alpha=4$. Latency introduced by the distance between $v_{P}$ and $v_{C}$ influences throughput only when $\alpha<4$. As such, when an accelerator tile has a FIFO buffer of four words, our guaranteed lower bound on throughput is reached and further increasing $\alpha$ can only increase best-effort throughput for this network size.

\subsection{Hardware Costs}

In this section we will evaluate our dual-ring implementation by looking at the hardware costs and putting them into perspective compared to the hardware costs of the rest of the design. A performance evaluation is done in a case study using a PAL video decoder which uses a single accelerator to replace four processors, as is explained in Section 7.1.3.

We implemented our dual-ring interconnect for an MPSoC with 16 CPU cores and one accelerator on a Xilinx ML-605 prototyping board. In Chapter 4 we showed that the ring scales linearly with the number of processor tiles and occupies only $\sim 2 \%$ of the total hardware costs in a homogeneous multi-core design.

When we consider our dual-ring interconnect, the NI comprises of the interface for both the data and credit ring and the shell which implements hardware flow control. In Table 5.2, we present the logic usage for our dual-ring interconnect normalized to the size of a single MicroBlaze processor. Note that the size of a MicroBlaze core depends not only on the features enabled for the processors in our design but also the total number of processors in that design ${ }^{1}$ : we compare the usage of our ring with the hardware costs of the $16 \mathrm{CPU}$ core design (which uses less logic per core than an eight or four core design). The ring routers for both the data and credit ring use $3.7 \%$ of the amount of logic needed for a single processor.

\footnotetext{
${ }^{1}$ We observed that the amount of required resources for a MicroBlaze with a specific set of features in an implemented design changes based on the size of the complete design. We assume that the extra effort needed to map increasingly bigger designs on an FPGA result in decreasing hardware costs per core. This seems counter-intuitive but was observed consistently over many instances.
} 


\begin{aligned} & \hline Component: Ratio: \\ & \hline Data Ring: $2.1 \% \\ &$ Credit Ring: $1.6 \% \\ &$ Shell: $4.8 \% \\ &$\hline\end{aligned}

TABLE 5.2 - Logic usage on a Virtex 6 FPGA of the dual-ring NI components compared to a MicroBlaze processor

As we want every processor to be able to communicate with an accelerator, the shell is part of the NI. The combination of both ring routers and shell results in a NI which is $8.5 \%$ of the size of a single processor.

\subsection{ConClusion}

In this chapter we presented extensions of our ring network from Chapter 4 required for efficient stream processor accelerator integration. Accelerator integration is facilitated by the addition of hardware flow control by means of a creditbased handshake. The first ring is used to transfer data. For hardware-based flow control a second unidirectional communication ring in the opposite direction of the first is introduced for the transfer of credits. Credits are only one bit wide and as such the credit ring has a lower hardware cost than the data ring.

Communication involving accelerators uses hardware flow control so stream processing accelerators see a streaming data channel without the need for using addresses. As such, stream processing accelerators have no notion of the underlying network. Communication between processor tiles is using software-based flow control using only the data ring.

The SDF model introduced in Chapter 4 was shown to be unsuitable for correctly modeling communication involving stream processing accelerators. Therefore, we derived a CSDF model which describes a hardware FIFO between two tiles. This model can be used to determine the required FIFO capacity in order to reduce the effects of network latency on application throughput.

The presented results indicate the potential of the dual-ring communication interconnect for use in low-cost heterogeneous medium-scale multiprocessor systems for real-time stream processing applications. 


\title{
Sharing of ACCELERATORS
}

\begin{abstract}
Stream processing accelerators are often applied in MPSoCs for software defined radios. Sharing of these accelerators between different data streams could improve their utilization and reduce thereby the hardware cost but is challenging under real-time constraints.

In this chapter we introduce entry-and exit-gateways that are responsible for multiplexing blocks of data over accelerators under real-time constraints. These gateways check for the availability of sufficient data and space at the output and thereby enable the derivation of a dataflow model of the application. The dataflow model is used to verify the worst-case temporal behavior based on the sizes of the blocks of data used for multiplexing. The use of this model for computing block sizes to determine multiplexing granularity is a novel application of dataflow analysis.

We demonstrate that minimum required buffer capacities are non-monotone in the block size. Therefore, an Integer Linear Program (ILP) is presented to compute minimum block sizes and sufficient buffer capacities (instead of minimum buffer capacities).
\end{abstract}

\subsection{INTRODUCTION}

Nowadays programmable signal processors are applied in SDRs. A hardware cost reduction can be achieved by specialization of the instruction set of these DSPs. However, the design effort and associated cost to create such processors and their accompanying compilation tool-chains can be significant.

Stream processing hardware accelerators can be an attractive alternative compared to the use of specialized DSPs, as was shown in Chapter 5. These accelerators can improve the performance and power-efficiency at the cost of reduced flexibility.

This chapter is based on work presented in [BHJD:6]. 
Sharing of accelerators between data streams could improve their utilization and reduce thereby hardware costs when their functionality is required multiple times in an application. Additionally, sharing of accelerators improves the flexibility of a single design as applications can use functionality provided by accelerators multiple times. Accelerators can be shared by different streams within one application or by data streams from different applications that are executed simultaneously on the multiprocessor system. However, sharing of accelerators is challenging under realtime constraints as a system implementation is required which can be captured in a temporal analysis model. With such an analysis model the minimum granularity at which blocks of data, i.e. the block size, must be multiplexed over the accelerators can be computed given the minimum throughput requirement of a stream of data of an application. Computation of the minimum granularity is complicated by the fact that a larger granularity amortizes accelerator reconfiguration overhead and thereby increases the throughput of one stream. However, this results also in a longer occupation of the accelerators and thereby reduces the throughput of other streams that share the same accelerator. As such, there is a mutual dependency between the minimum block sizes for different streams. Another complicating factor is that intuitively a smaller block size should result in a smaller buffer capacity but this is not the case in general because the required buffer capacities are a nonmonotonic function in the block sizes. As a result the total required memory size for buffering is a non-monotonic function in the block size as well.

In this chapter we present an approach for sharing accelerators under real-time constraints in a multiprocessor architecture for stream processing applications. Sharing of stream processing accelerators is enabled by multiplexing data streams using so-called entry- and exit-gateways. Streams are considered for execution in a round-robin fashion; when a stream is not eligible it is skipped and the next stream is considered. These gateway pairs check for sufficient input data and output space at respectively the input and output of a chain of accelerators such that the minimum throughput can be derived using a CSDF model [7]. An abstraction of this CSDF model is created in the form of a single actor SDF model. This SDF model enables the derivation of an ILP for the computation of the minimum granularity at which blocks of data from the streams must be multiplexed over shared accelerators.

Reduction in hardware costs has been evaluated by creating a multiprocessor system with shared accelerators that is implemented on a Virtex 6 FPGA. This system has been evaluated using a real-time PAL stereo audio decoder application, which is presented in Chapter 7 .

The outline of this chapter is as follows. The basic idea behind our approach is described in Section 6.2. The derivation of the temporal analysis models in the form of a CSDF and the more abstract SDF model are described in Section 6.3. Evaluation of our system in terms of hardware costs is described in Section 6.4 and potential future improvements are explained in Section 6.5. The conclusion is stated in the last section. 


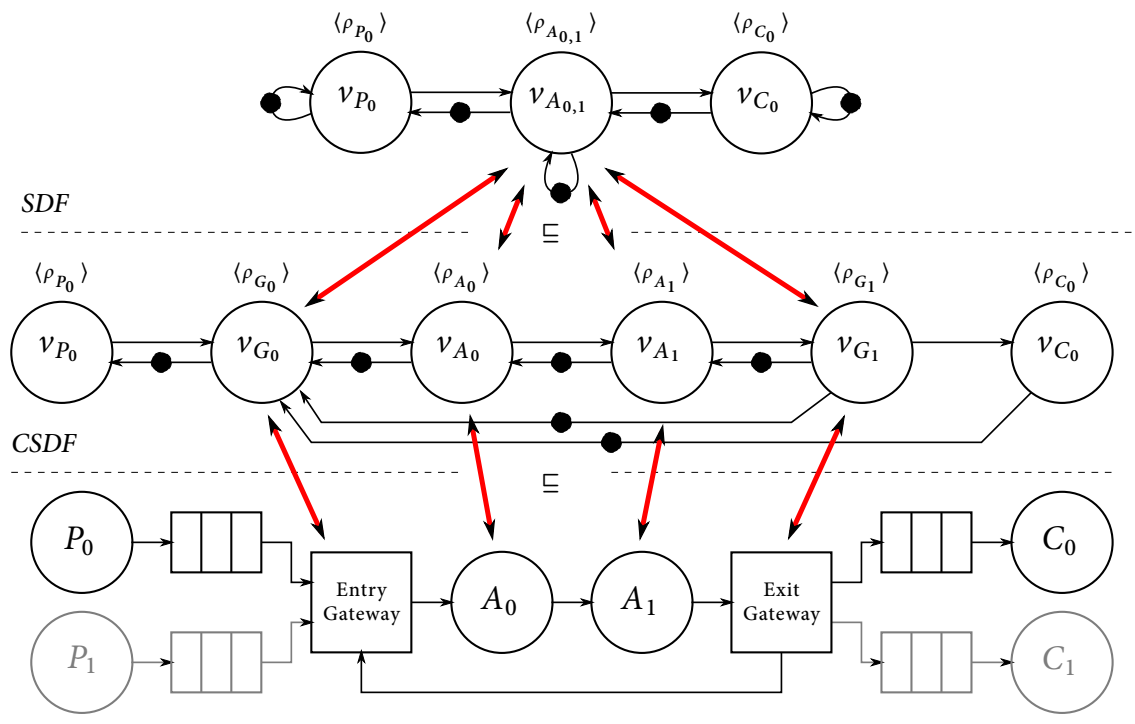

Reality

FIGURE 6.1 - Basic idea for sharing accelerators

\subsection{BASIC IDEA}

In this section we present a relation between our architecture and the dataflow models used for the derivation of the minimum block size.

An overview of our hardware architecture has been presented in Chapter 3 and depicted in Figure 3.1. In this architecture an entry-gateway hardware block is responsible for scheduling blocks of data from different streams over a chain of accelerators. Streaming of a block of data is only allowed if:

1. the exit-gateway has indicated that all data in the chain of accelerators belonging to the previous block has been processed so a context switch can be performed, and:

2. enough space is available at the output buffer such that a consuming task can receive the next block of data without having to stall for additional space in this buffer

A CSDF model suitable for temporal analysis can be created for this hardware, which is shown in Figure 6.1. This figure shows that one CSDF model per stream can be created despite that accelerators are shared. This CSDF model is a conservative abstraction of the hardware implementation. We will show that the minimum throughput can be derived using this model.

We will use the symbol $\subseteq$ to indicate that the CSDF model is a conservative abstraction of the hardware. As such, the hardware is a temporal refinement of this model, 
according to the-earlier-the-better [85] refinement theory. This theory is applicable if the application is functional deterministic and for each component it holds that:

$$
\forall i, a(i) \leq \hat{a}(i) \Rightarrow \forall j, b(j) \leq \hat{b}(j)
$$

This equation states that an earlier arrival of the $i$-th input token at time $a(i)$ in the refined model compared to the arrival of the corresponding token in the abstraction at $\hat{a}(i)$ should not result in a later production of the token by the refined model at $b(j)$ compared to production of the corresponding $j$-th token by the abstraction at $\hat{b}(j)$.

This refinement theory can be altered [31] to be applied to models where the output $b(k)$ for an arbitrary $k$ is used as input $a(k+l)$ with $l>0$ resulting in a circular dependency. If such a circular dependency is present, it is not possible without further evaluation of the model to determine if the relation from Equation 6.1 holds for all $a(i)$ as these will depend on $b(j)$. This can be solved by iteratively computing schedules for the model for a set of inputs and verifying that the relation holds for $\forall i<m$ and $\forall j<n$, with $m$ and $n$ being incremented every iteration, as is described in [31]. To denote that we compare arrival times up to and including the $i^{\text {th }}$ token we use the notation from [31]. The earlier-the-better refinement theory thus becomes:

$$
\forall i, a\langle i\rangle \leq \hat{a}\langle i\rangle \Rightarrow \forall j, b\langle j\rangle \leq \hat{b}\langle j\rangle
$$

This equation can be generalized for components with multiple inputs and outputs. If the equation holds we say that component $C$ refines component $C^{\prime}$ and this is formalized as $C \sqsubseteq C^{\prime}$. A component can be an actor, its corresponding task or subgraphs of actors or tasks. If the refinement relation holds per component then it holds also for a complete graph of components. This means that the worst-case throughput and latency of the refined graphs is always better than the model being refined, i.e. the model obtained by abstraction. As such temporal guarantees concerning maximum token arrival times derived for the abstract model are always valid for the refined model and this also holds for subgraphs of these models. As a result, it is sufficient to show that a schedule of the abstract model exists that satisfies the temporal requirements.

We can therefore determine the minimum throughput by creating an admissible schedule for the CSDF graph at design-time. In an admissible schedule actors do not fire before they are enabled. Actors are enabled if sufficient tokens are available in their input queues. During the construction of this schedule we use worst-case firing durations of the actors. These firing durations are equal to the maximum durations between arrival of the data enabling a task such that it becomes eligible for execution and the actual production of data by this task. Tasks can be executed by accelerators or by processors.

The block size determines the number of samples to be processed by the accelerators before a block of another stream can be processed. To derive the block size 


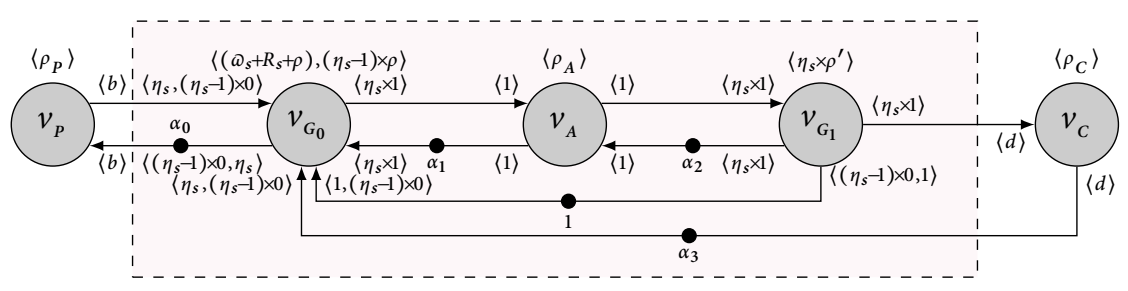

FIGURE 6.2 - CSDF model of accelerator multiplexing for a data stream $s$

using the dataflow model we cannot make use of MCM analysis techniques [73] as these analysis techniques require that expansion into an HSDF graph is possible [7]. However, an HSDF graph with a fixed topology cannot be derived as the block size remains a variable parameter in the CSDF model. Instead of computing the MCM we construct a schedule that is parameterized in the block size.

We will show that a more compact parameterized SDF model can be created from this CSDF model with a minimum loss of accuracy. As such, the CSDF model is a refinement of the SDF model, as is indicated in Figure 6.1. Due to transitivity of the ᄃ relation we can conclude that also the hardware is a refinement of this SDF model. We will show that this SDF model is suitable for the derivation of the minimum block size such that the throughput constraint of the application is satisfied despite overhead of saving the state and flushing data out of the FIFO buffers between the accelerators.

\subsection{Dataflow Model}

In this section the CSDF model of our sharing approach for accelerators and its SDF abstraction are explained in detail. We will partially reiterate the definition from Section 5.3 and define a CSDF notation which is in line with the classic definition from [7] (which was not used in Chapter 5). A detailed CSDF model will be presented for a single data stream using a shared accelerator followed by its abstraction into a SDF model.

\subsection{1 (C)SDF}

CSDF extends SDF, as was introduced in Section 5.3, by introducing the concept of phases. Each CSDF actor has by definition an implicit self-edge with one token ${ }^{1}$. Furthermore, each actor has a cyclic behavior during which its phases are fired. The firing duration for every phase $p$ is denoted as $\rho_{v}[p]$. Both firing durations and quanta are expressed as a list of values with a length equal to the number of phases of the corresponding actor. To denote a parametric list of quanta for each phase of a CSDF actor, we use the notation: $\langle z \times 1,0\rangle$, denoting $z$ phases with quanta 1 followed by one phase of quanta 0 .

\footnotetext{
${ }^{1}$ Note that this differs from the CSDF model definition used in Chapter 5
} 


\subsubsection{CSDF MOdEL}

In our approach gateways are used for multiplexing of data streams over a set of accelerators. For each stream that is multiplexed over the accelerators a separate CSDF model is created. A CSDF model for one such stream is shown in Figure 6.2. To keep the figure concise we assume, without loss of generality, that only one accelerator is shared. This accelerator is modeled with actor $v_{A}$. The values of $\alpha_{1}$ and $\alpha_{2}$ are two tokens and are equal to the capacity of the buffers in the network interfaces.

In the CSDF model, the entry-gateway is modeled by actor $v_{G_{0}}$ and the exit-gateway is modeled by $v_{G_{1}}$. The first phase of $v_{G_{0}}$ can not start before $v_{G_{1}}$ has produced a token on the edge $\left(v_{G_{1}}, v_{G_{0}}\right)$ which indicates that the previous block of data has been processed. Additionally, $v_{C}$ must have produced $\eta_{s}$ tokens to indicate that $\eta_{s}$ locations are available in the input buffer of $v_{c}$. Furthermore, at least $\eta_{s}$ tokens have been produced by actor $v_{P}$ before the first phase of $v_{G_{0}}$ can start.

Actor $v_{G_{0}}$ produces $\eta_{s}$ tokens, one in each phase. All phases denote the transfer of a sample to the shared accelerator $v_{A}$. Actor $v_{A}$ will consume each token and produce a token for the exit gateway actor $v_{G_{1}}$. After $v_{G_{1}}$ has produced $\eta_{s}$ tokens for actor $v_{C}$, with each token modeling an output sample from the shared accelerator to the consumer, it produces a token to notify $v_{G_{0}}$ that the pipeline is idle.

The time that other streams might use the accelerators before stream $s \in S$ can be processed, denoted by $\omega_{s}$ is a function in the block sizes of all other streams and is included in the firing duration of the first phase of actor $v_{G_{0}}$. The set $S$ contains all streams multiplexed by an entry-/exit-gateway pair over a set of accelerators. The time $R_{s}$ required to reconfigure the accelerators for stream $s$ is also included as well as the time it takes for $v_{G_{0}}$ to copy a sample, which is denoted by $\rho$. As such, the firing duration of $\rho_{G_{0}}[0]$ becomes:

$$
\rho_{G_{0}}[0]=\omega_{s}+R_{s}+\rho
$$

The durations of all other phases of $v_{G_{0}}$ are $\rho$. Similarly, the time needed to copy a data element by $v_{G_{1}}$ is denoted by $\rho^{\prime}$.

Given the CSDF model in Figure 6.2 we can construct an execution schedule for the gateways and accelerator that process a single stream $s$ under the assumption that the pipeline was initially idle, thus $\omega_{s}=0$, as shown in Figure 6.3. This schedule is parameterized in $\eta_{s}$ and shows that after $\eta_{s}$ firings of all actors a complete block has been processed and is ready to be consumed by $v_{C}$. From this schedule we can conclude that the total processing time of one block of $\eta_{s}$ samples for data stream $s$ is $\tau_{s}$ time, which is upper bounded by $\hat{\tau}_{s}$ :

$$
\tau_{s} \leq \hat{\tau}_{s}=R_{s}+\left(\eta_{s}+2\right) \cdot \max \left(\rho, \rho_{A}, \rho^{\prime}\right)
$$

According to Equation 6.4, $\tau_{s}$ consists of the reconfiguration time plus the time needed to process $\eta_{s}$ samples and the time needed to empty the pipeline of gateways and accelerator(s). The maximum of the firing durations of the gateways and 


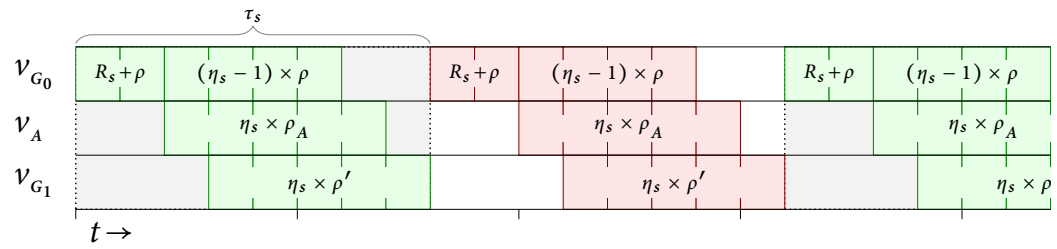

FIGURE 6.3 - Execution schedule of stream $s$ processing blocks of $\eta_{s}$ samples

the accelerator(s) is taken because the actor with the largest firing duration in the chain determines the time to process a sample.

Streams are multiplexed in a RR fashion by the entry-gateway. By applying the theory presented in [55] we can conclude that when a block of data for stream $s$ is queued, in the worst-case it has to wait $\omega_{s}$ time. This is equal to the processing duration of a block of data for every other stream that uses the same accelerator, before the block of stream $s$ is processed. Therefore $\omega_{s}$ is bounded from above by:

$$
\omega_{s} \leq \hat{\omega}_{s}=\sum_{i \in S \backslash s} \hat{\tau}_{i}
$$

As a result the maximum time $\gamma_{s}$ it takes before a block of queued samples of stream $s$ is processed, is equal to the sum of the processing time of one block of data for all streams sharing that accelerator:

$$
\gamma_{s}=\hat{\omega}_{s}+\hat{\tau}_{s}=\sum_{i \in S} \hat{\tau}_{i}
$$

\subsubsection{Single SDF ACTOR MOdEL}

The detailed CSDF model inside the dashed box in Figure 6.2 can be abstracted into a single actor SDF model as shown in Figure 6.4. The firing duration of this SDF actor is $\hat{\gamma}_{s}$. There is hardly any loss in accuracy because processing of the next block cannot start before the previous block has been processed completely. The only loss in accuracy compared to the CSDF model is caused by the fact that the SDF actor will atomically produce all tokens when the actor finishes while in the CSDF model tokens for $v_{C}$ are produced during firing of $v_{G_{1}}$ and will therefore arrive earlier.

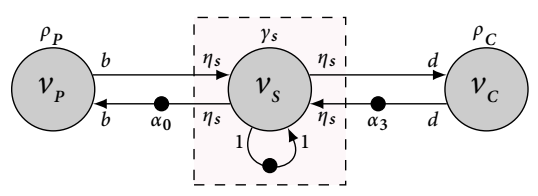

FIGURE 6.4 - SDF abstraction for a data stream $s$ over an accelerator $v_{s}$ 
This means that the SDF model is more pessimistic than the CSDF model and as such, according to the-earlier-the-better refinement, any throughput guarantees from the SDF model will hold for the CSDF model and hardware as well.

\subsubsection{Minimum Throughput Verification}

When block sizes are specified by the designer, the firing duration for actors $v_{s}$ denoting shared accelerators can be determined using Equation 6.6. The quanta of $v_{s}$ are equal to the block size $\eta_{s}$, except for the quanta of the self-edge. The resulting graph is an SDF graph and the minimum throughput and the minimum required buffer capacities given a throughput constraint can be determined using existing SDF techniques [22].

\subsubsection{NON-MONOTONE BEHAVIOR}

When block sizes are not specified by the designer, they have to be computed for the application. This computation is difficult as minimum buffer capacities are not monotone in the block size. We will illustrate this with an example using the model from Figure 6.5.

We can determine for every value of $\eta_{s}$ what the minimum required buffer capacities are using an existing SDF technique [22] to obtain maximum throughput. When we do this for the model from Figure 6.5 for $\eta_{s}=2$ and $\eta_{s}=5$, we see that from Table 6.1 the small block size requires a larger buffer capacity than the larger block size. However, when considering the required buffer capacities needed for $\eta_{s}=1$ and $\eta_{s}=2$, the opposite is true. As such, the minimum buffer capacities are not monotone in the block size and thus using the smallest possible block size does not result in the smallest possible buffer capacities in general. A similar issue occurs for minimum buffer capacities under a throughput constraint.

\subsubsection{Computing Block Sizes}

Our SDF model can be used to create an ILP to compute minimum block sizes and sufficient buffer capacities. Note that an over-approximation is used to create a linear program. This ILP is correct for graphs with a topology as shown in Figure 6.4. In such a graph block size $\eta_{s}$ is computed for an actor which is only part of the cycle denoting the FIFO channel to and from that actor and no other cycles. Topologies where such an actor is part of more cycles have a non-linear solution and as such the presented ILP will not be applicable.

For every stream $s$ in an application a certain minimum throughput $\mu_{s}$ is specified, expressed in samples/s, which can be derived from the throughput constraint of the application. If we consider the SDF model from Figure 6.4 and assume that $\alpha_{0}$ and $\alpha_{3}$ are sufficiently large to make the self-edge on $v_{s}$ critical, the requirement on the minimum throughput of stream $s$ can be described by:

$$
\frac{1}{\gamma_{s}} \cdot \eta_{s} \geq \mu_{s}
$$




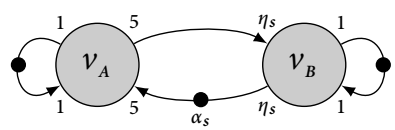

FIgURE 6.5 - SDF model

To find the minimum block size for every stream multiplexed over a specific accelerator we have to derive $\eta_{i}$ which satisfies Equation 6.7 for every stream $i \in S$. The values of $\eta_{i}$ can be computed with the following ILP which is created by substitution of $\gamma_{s}$ - as defined in Equation 6.6 - in Equation 6.7.

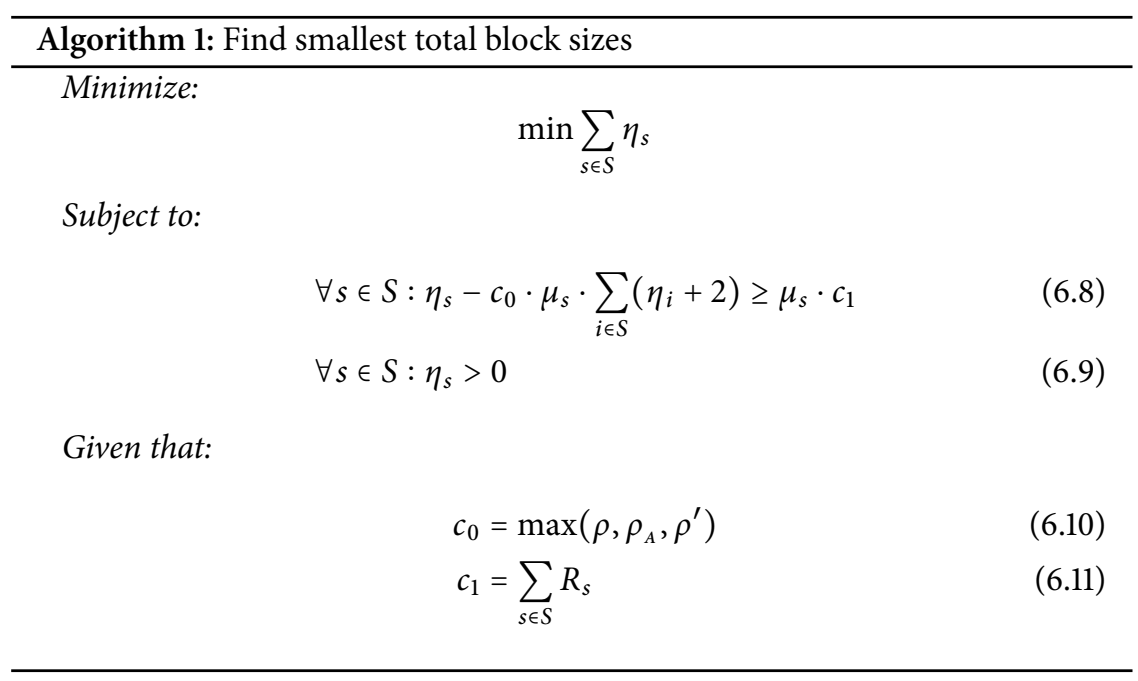

After finding the smallest block sizes, a standard algorithm for the computation of the minimum buffer capacities [22] can be used.

To find the optimal block sizes resulting in the smallest buffer capacities, a computational intensive branch-and-bound algorithm can be used. This algorithm has to verify whether the throughput constraint of every stream is satisfied for every possible block size and must compute the accompanying minimum buffer capacities to find the total minimum buffer capacity.

\begin{tabular}{r|ccccc}
$\eta_{s}$ & 1 & 2 & 3 & 4 & 5 \\
\hline $\min \alpha_{s}$ & 5 & 6 & 7 & 8 & 5
\end{tabular}

TABLE 6.1 - Buffer capacities for various $\eta_{s}$ 


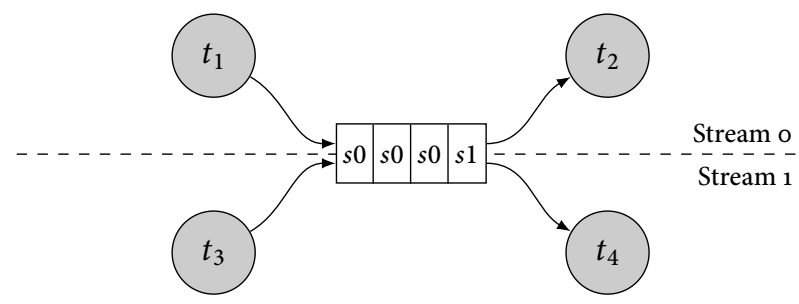

FIGURE 6.6 - Task graph showing shared FIFO sharing

\subsubsection{Check for Space}

As described before, in our model we not only have to check for space in the FIFO of the first stream processing accelerator but also in the buffer between the exitgateway and the consumer. We will now justify the need for this check.

In Figure 6.6 a task graph with two pairs of producers and consumers is depicted sharing a FIFO. Such a FIFO is found between the gateways and accelerators in our architecture. Task $v_{1}$ and $v_{2}$ produce and consume data, denoted by $s 0$, for stream 0 . Tasks $v_{3}$ and $v_{4}$ do the same for stream 1 where data for that stream is denoted by $s 1$.

Dataflow models like SDF define that the moment of production of a token is the same instant a token arrives at the consumer. When this FIFO is shared between streams, for example when streams are multiplexed, tokens from another stream can influence when produced tokens arrive at the consumer because of head-ofline blocking. This is not allowed in SDF and causes that the-earlier-the-better refinement is not applicable.

In the worst case, an indefinite stall of one of the consuming actors from Figure 6.6 will stall the other consuming actor indefinitely as a single token for the stalled actor can block the FIFO.

Our sharing approach provides mutual exclusivity: when a stream $s$ wants to use the FIFO it needs to wait for the current stream to be emptied from the FIFO. When this happens, a token produced by $s$ will immediately be available at the FIFO output, satisfying the conditions of the refinement theory.

\subsection{Evaluation}

In this section we will present the hardware costs of the gateways used to implement our sharing implementation. We will briefly introduce the demonstrator used to evaluate the hardware reduction obtained due to accelerator sharing. The hardware costs of our gateways and accelerators are presented which demonstrates the benefits in terms of hardware costs and utilization of our sharing approach.

As described in Section 3.5, our entry-gateways are implemented using a MicroBlaze CPU. As such, the hardware costs of an entry-gateway consists mostly of a 


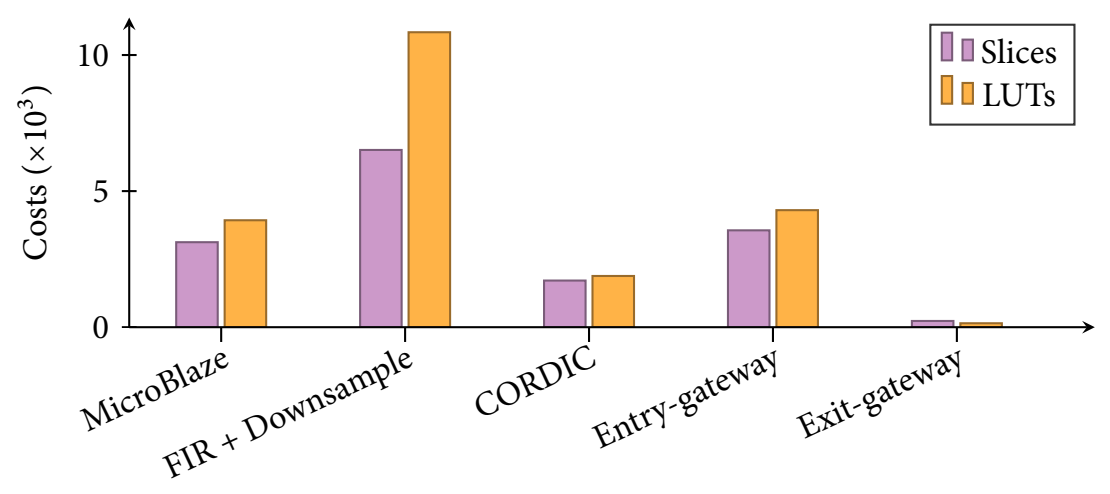

FIGURE 6.7 - Hardware costs of various components in a Virtex 6 FPGA

single MicroBlaze processor, as was depicted in Figure 3.4. Therefore, the hardware costs of an entry-gateway are slightly larger than a processor, as can be seen in Figure 6.7. The exit-gateway is fully implemented in hardware and its costs are also depicted in Figure 6.7.

As such, the hardware costs for a complete entry and exit pair consists mainly of the costs of one MicroBlaze, the DMA engine and the exit-gateway. These costs are shown in Table 6.2.

In order to evaluate the obtained hardware cost reductions, a case study was performed where a PAL stereo audio decoder application was implemented. Further details about this application will be introduced in Chapter 7. This decoder requires a 33 taps complex FIR filter with built-in programmable down-sampler and a CORDIC block, each four times. Without accelerator sharing this application would require four instances of each accelerator type. This would result in a lot

\begin{tabular}{|c|c|c|c|}
\hline Component & Slices & LUTs & \\
\hline \multirow{3}{*}{$\begin{array}{r}\text { Entry- + Exit-gateway } \\
\text { FIR + Down-sampler } \\
\text { CORDIC }\end{array}$} & 3788 & 4445 & \\
\hline & 6512 & 10837 & \\
\hline & 1714 & 1882 & \\
\hline \multicolumn{4}{|l|}{ Non-shared vs Shared } \\
\hline \multirow{3}{*}{$\begin{array}{r}4 \times \text { FIR }+4 \times \text { CORDIC } \\
\text { Gateways }+ \text { FIR }+ \text { CORDIC } \\
\text { Savings }\end{array}$} & 32904 & 50876 & \\
\hline & 12014 & 17164 & - \\
\hline & $\begin{array}{r}20890 \\
(63.5 \%)\end{array}$ & $\begin{array}{r}33712 \\
(66.3 \%)\end{array}$ & \\
\hline
\end{tabular}

TABLE 6.2 - Hardware costs and savings for a demonstrator implemented in a Virtex 6 FPGA 
of duplicated hardware which would also have a low utilization. In this case, by having just one of each type of accelerator, sharing implemented by the gateways removes the need for duplicate logic. The demonstrator still meets its throughput constraint while more than $63 \%$ of the hardware costs of the accelerators could be saved.

\subsection{Future IMProvements}

While our current sharing approach is sufficient for the case studies we present in this thesis, there remain further options to improve the design based on observations from these case studies.

\subsubsection{Gateway Cost Reduction}

We expect that our sharing approach could be implemented completely in hardware, resulting in a DMA-like entry-gateway which will be considerably smaller than a MicroBlaze CPU. The resulting gateway will additionally be able to reconfigure shared stream processing accelerators much faster than the software-based entry-gateway. This reduction in reconfiguration time will reduce latency for all data streams using shared accelerators, increase throughput and will allow to use smaller block sizes used for multiplexing.

\subsubsection{HierarchicAl Rings}

Accelerators that are to be shared between multiple data streams will not be sending or receiving data directly from processor tiles. Additionally, processor tiles currently only support receiving and sending a single data stream when communicating with accelerators.

These facts, combined with the fact that shared accelerators only communicate with gateways results in the idea for a multi-level hierarchical interconnect. In this design, a group of accelerators is managed by a pair of gateways as was presented previously. However, these accelerators have a dedicated ring per group where the gateways are also connected to a global ring. This idea is illustrated in figure 6.8.

This design would remove the need for the dual-ring interconnect for the global ring as processor tiles and gateway tiles use our C-FIFO implementation for communication channels. As such, we feel this approach would lower hardware costs, reduce the number of concurrent streams as there are now multiple rings and reduce the maximum latency and increase minimum throughput using the previously presented arbitration policy.

\subsection{Conclusion}

In this chapter we presented an approach for sharing stream processing accelerators by means of gateways in a heterogeneous multi-core stream processing ar- 


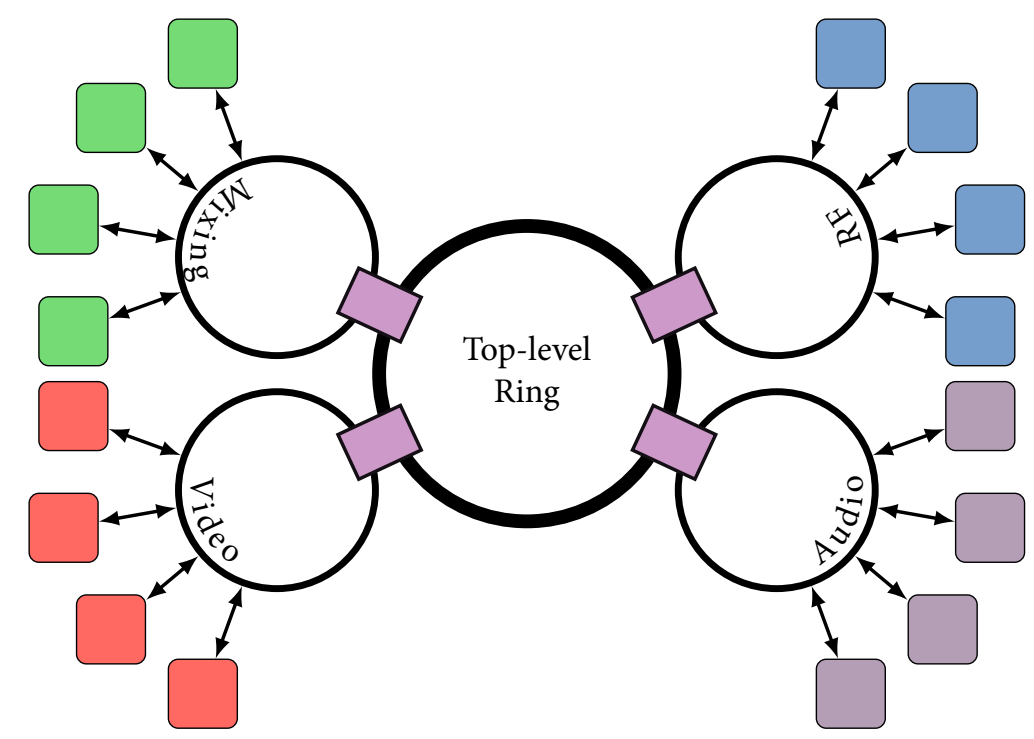

FIGURE 6.8 - Example of an hierarchical ring with sub-rings grouping functionality

chitecture under real-time constraints. The hardware overhead introduced by the entry- and exit-gateways is reduced by multiplexing multiple data streams over various accelerators. This reduces the need for duplicated functionality and improves utilization of accelerators.

Sharing accelerators also improves the flexibility of a single design as the functionality provided by stream processing accelerators can be used multiple times in applications. This means that a specific instance of an architecture can be used for a wide range of applications as the physical number of stream processing accelerators providing a certain functionality is not a restriction in any application to be executed. In one of our case studies we implemented a real-time GMSK radio decoder application which could be evaluated in multiple configurations using shared stream processing accelerators on a single instance of our architecture.

We presented a CSDF temporal analysis model which can be abstracted into a single SDF node model to describe a gateway and a chain of accelerators which are shared between multiple streams. The corresponding model can be used to check if throughput constraints are satisfied when block sizes are specified. An ILP is presented to compute minimum block sizes under a throughput constraint. However, we showed that minimizing the block sizes reduces the required buffer capacities but does not necessarily result in the minimal buffer capacities due to the nonmonotonic relation between block sizes and buffer capacities.

Any reduction in the hardware costs depends on the application and the accelerators that are to be shared. In one of our case studies we used a real-time PAL stereo 
audio decoder application - which is presented in Chapter 7 - which saw an improved utilization of the accelerators and a 63\% reduction in hardware compared to a design without shared accelerators. 


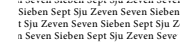

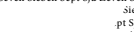 \\ Case Study
}

\begin{abstract}
The proposed architecture is evaluated using real-time stream processing applications.

In this chapter we will describe these applications and the obtained evaluation results. The three applications we have used for the case studies are PAL video decoding, PAL audio decoding and GMSK decoding. These applications are used to evaluate the data ring using software based FIFO communication, the dual ring using hardware based FIFO communication, and the gateways for sharing accelerators between multiple data streams under real-time constraints.
\end{abstract}

The results indicate that our dual-ring interconnect can be an attractive solution for medium scale heterogeneous multi-core architectures that execute real-time stream processing applications. Our approach for multiplexing of real-time data streams over stream processing accelerators improves the utilization of accelerators and lowers hardware costs by removing the need for multiple accelerators of the same type.

In this chapter we present the case studies that have been performed in order to evaluate the architecture. With each case study, a specific aspect of the proposed architecture is evaluated.

The support for accelerators in the Omphale compiler is incomplete. Therefore, the applications written for our case studies are described using $\mathrm{C} / \mathrm{C}++$. However, in each application a top-level program description is used which is similar to a description which could have been generated by the Omphale compiler.

In our experiments we often have tasks that are limited in their throughput by the amount of available processing power. We call such tasks computationally bounded.

Parts of this chapter are based on [BHJD:2, 4, 6]. 
Throughput for such tasks is increased by assigning more processing time, limited to the point where only one such task is executed on a single processor.

For the first case study we implemented a PAL television decoder application. This video decoder was implemented as a task graph where each task in the algorithm was implemented in software where some of the computationally bounded tasks without state were duplicated and mapped to multiple processors to improve application throughput. This case study focuses on testing both software and hardware based FIFO communication and demonstrates the performance benefits of using accelerators in stream processing applications. We also demonstrate the ease of using accelerators because a simple switch can be made between executing a task on a processor or on an accelerator, assuming the same functionality is available on both.

In the second case study, sharing of accelerators is demonstrated using the stereo audio decoding part of a PAL decoder application. Because the decoding of the two audio channels is very similar, both channels require the same demodulation. Therefore, the demodulation is implemented with two stream processing accelerators which are used to demodulate both audio channels.

For our third case study a GMSK decoder with Viterbi error correction was implemented. Such a decoder is used in Bluetooth Low-Energy receivers. The use of a convolutional decoder is proposed in the Bluetooth Low-Energy Long-Range standardization committee to improve the sensitivity of the receiver. For this case study, we implemented a Viterbi Algorithm with support for so-called soft-decision input. Soft-decision means that an estimate is provided of the correctness of a symbol while hard-decision means that a symbol is chosen and any information about the probability of correctness is discarded. The use of soft-decision input can improve the Bit Error Rate (BER) over hard-decision input [26].

For the case studies multiple instances of our architecture have been implemented on a Xilinx ML-605 development board which contains a Virtex 6 FPGA. Radio signals are received using an Epiq FMC-1RX [17] direct conversion receiver frontend (FE).

\subsection{PAL Television Decoder}

In this section we describe the used hardware and software for evaluation and mapping of video and audio decoding applications targeting the PAL broadcasting standard. This analogue standard was chosen because video and audio are transmitted separately. Decoding can be done at a range of sample rates where higher quality output is produced at higher sample rates. This usually does not hold for digital radio standards which have to be decoded at one specific sample rate.

The hardware costs, as presented in Section 4.6 and Section 5.4, from the dual-ring interconnect are evaluated by comparing them to the costs of the complete system. We evaluate the suitability of the interconnect for the implemented video and audio 
decoding applications based on the obtained performance gains due to the use of accelerators. We will also evaluate the utilization of the bandwidth provided by the ring.

\subsubsection{PAL Video Decoder}

In order to evaluate the performance of the proposed architecture we implemented a PAL decoder in software on a 16 core design. The PAL standard describes a field interlaced, 25 frames per second color video signal, usually in combination with FM modulated audio [39].

Our decoder is designed for the PAL-B, PAL-G, PAL-H and PAL-I broadcast standards. A PAL-B broadcast uses channels occupying $7 \mathrm{MHz}$ bandwidth while the PAL-G, PAL-H and PAL-I broadcasts use channels that are $8 \mathrm{MHz}$ apart. The video transmission occupies a $5 \mathrm{MHz}$ bandwidth and consists of an Amplitude Modulated $(A M)$ luminance signal - usually denoted by $\mathrm{Y}$ - and a quadrature modulated color difference signal - consisting of R-Y and B-Y - which is modulated at $4.43 \mathrm{MHz}$ from the luminance carrier frequency. In a PAL signal, the color signal overlaps with the luminance signal.

During color decoding, the color signal has to be removed from the video signal to restore the original (monochrome) luminance signal. If the color signal is not filtered out, it will be visible as high frequency distortions in the monochrome video at high sample rates. When the sample rate is not high enough or only monochrome video is to be decoded, the color signal can be ignored as the lowpass filter before the analogue to digital converter will remove high frequency components including the color signal. The optional decoding and filtering of the color component in the video broadcast is a feature from the PAL standard [39] in order to be backwards compatible for monochrome TVs.

Our demonstrator contains a monochrome video decoder which ignores the color component and uses the complete video signal as a luminance video source. This AM modulated luminance signal contains 625 horizontal scan lines per frame, 25 frames per second where each frame is transmitted as two consecutive interleaved fields. As such, the vertical resolution is fixed to the number of lines whereas the horizontal resolution is directly related to the sample rate. By sampling the video broadcast at a relatively low speed compared to the color carrier and by applying an appropriate low-pass filter, we can eliminate interference from the color signal. Lowering the sample rate comes at the cost of lowering the horizontal resolution of the video frames. When the horizontal resolution is not high enough due to the chosen sample rate to produce a native $4: 3$ aspect ratio - as defined by the PAL standard - it has to be upscaled in one dimension to obtain the correct aspect ratio.

The video signal is inverted before transmission and can be reconstructed at the receiver by simply inverting the signal again. Every line is separated by a synchronization pulse which uses a lower signal value than the rest of the luminance signal, as can be seen in Figure 7.1 and as marked in Figure 7.2. For our decoder we nor- 
malize the signal input range so that the baseband signal ranges from 0.0 to 1.0 (or $100 \%$ intensity) where 0.3 and lower are used for line synchronization pulses and 0.3 to 1.0 is used for the monochrome video signal. Using this normalization, lines in a field are separated by a small synchronization pulse with a signal value around 0.0 intensity.

As mentioned before, each frame consists of two interleaved fields. All fields are separated by a number of special synchronization pulses, as is shown in more detail in Figure 7.1. These pulses consist of a square wave between the signal level 0.0 and 0.3 in the normalized range.

A new field is denoted by two and a half lines containing five synchronization signals where each signal consists of a long $(27.3 \mu \mathrm{s})$ low pulse, followed by a short $(4.7 \mu \mathrm{s})$ high pulse. These pulses can be seen in Figure 7.1 on line 1, 2 and 3 and on line 313, 314 and 315. By detecting this sequence in the signal stream, the decoder can lock on to the start of a field. Note that the position of the first synchronization signal differs by half a line between fields. Therefore, the field type has to be detected to correct for this timing offset.

Field synchronization detection is necessary to correctly deinterlace the fields and can be implemented in various ways. We implemented field synchronization detection in such a way that it does not require prior timing information. This is achieved by searching for the five low-high synchronization pulses to give an estimate of the start of a field, as is indicated by the red arrow for both field types
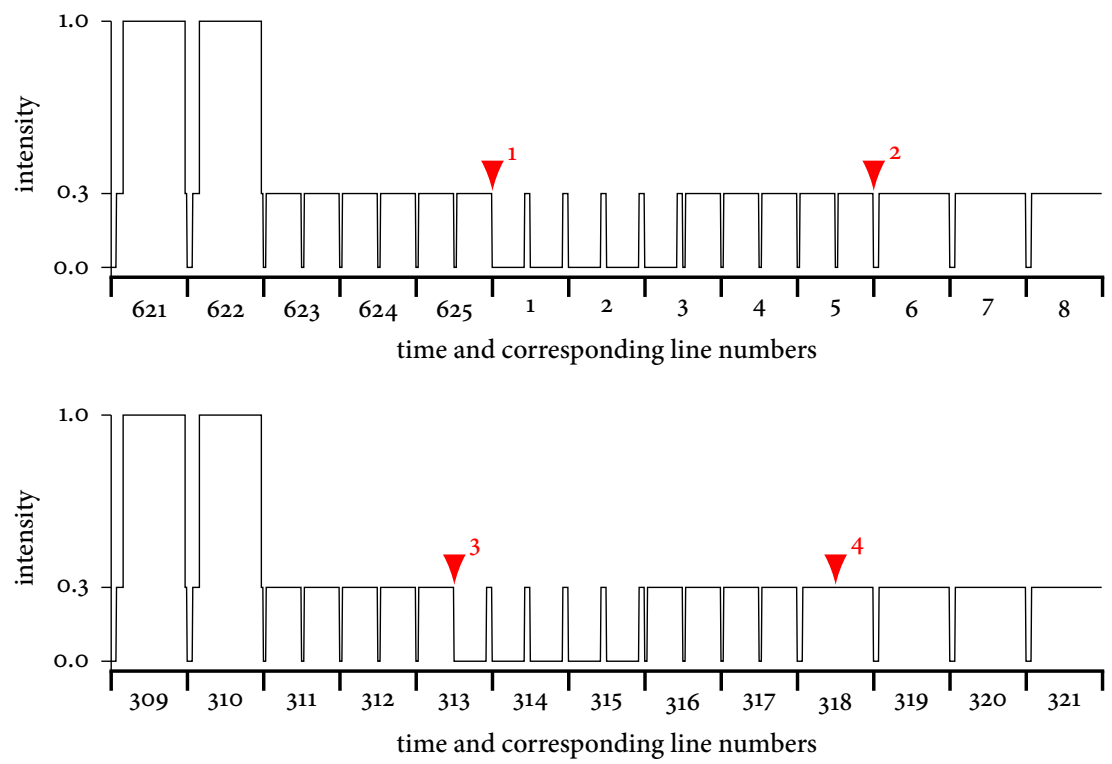

FIGURE 7.1 - ITU-R 470-6 PAL timing diagram for field synchronization 


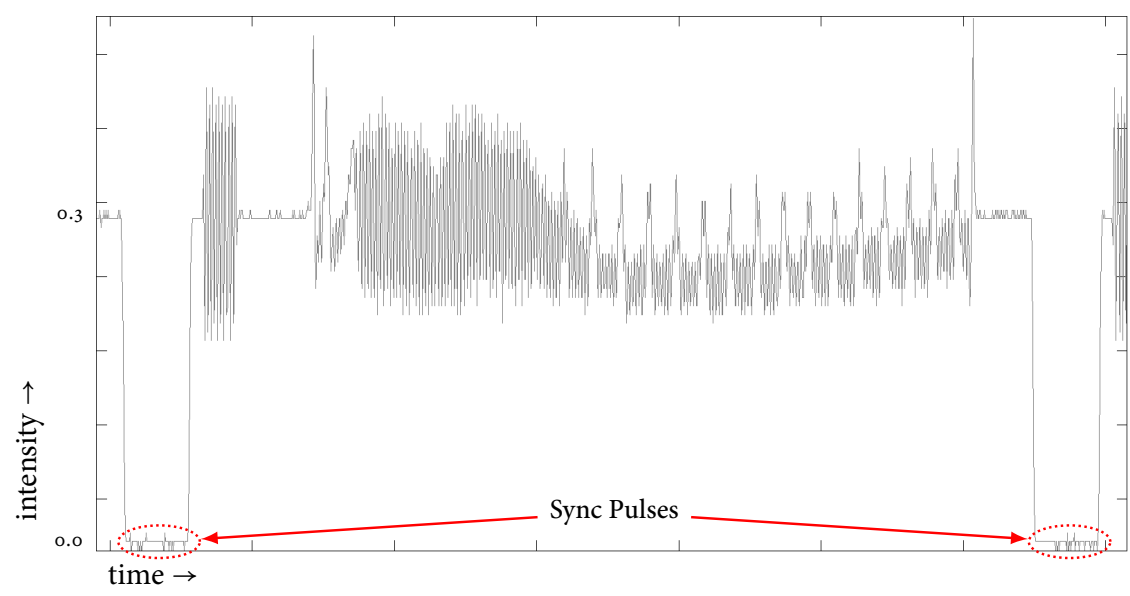

FIGURE 7.2 - Complete PAL line with two low valued synchronization pulses

denoted by 1 and 3 in Figure 7.1. After finding this point in time, the field type can be determined by inspecting a known offset from the detection point, as is indicated by the arrows 2 and 4 in Figure 7.1 for both types: the first field will contain a line synchronization pulse while the second field will have a line containing black video material at that point. When the field type is determined, the half line offset for the frame can be corrected in order to determine the correct start of horizontal video lines.

After the field synchronization has been found and the type has been detected, the deinterlacer will use this information to buffer lines until both an even and odd field have been received. When a frame has been reconstructed by the deinterlacer, it can be displayed.

\subsubsection{Demodulating Video}

In this case study, the data ring of the dual-ring interconnect is used and all tasks are implemented in software.

Our parallel decoding algorithm is depicted as a task graph in Figure 7.3. The actors in this graph correspond with software tasks where each task is allocated to a dedicated CPU. While our system contains support for multiple tasks per processor tile, in this experiment we allocated one task to each CPU.

After acquiring I/Q samples at baseband from the radio front-end (ADC) in software - where the front-end is used as a slave peripheral for the processor tile executing the "ADC" task, the AM signal is reconstructed from the magnitude of the I/Q samples. The resulting intensity of the luminance signal is used in the field synchronization detection. At the beginning of a field, the field type is detected: one field type is used for even lines, the other for odd lines. After the field type detec- 


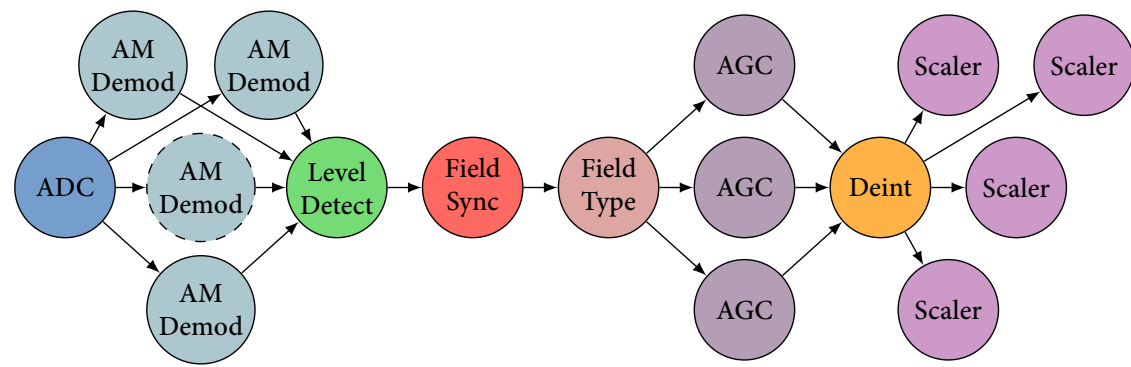

FiguRE 7.3 - Task graph of our PAL decoder application

tion, the signal is normalized to obtain a range suitable for displaying which is then adjusted with contrast (signal scaling) and brightness (signal offset) settings. The field alignment is used to calculate the start of each line and is used by the deinterlacer task to generate complete $2 \mathrm{D}$ frames. When a frame is complete, a one dimensional scaler is used to scale the horizontal resolution to obtain a 4:3 display ratio. A resulting video frame is shown in Figure 7.4 which corresponds with the input signal that is shown in Figure 7.2.

For the detection of field synchronization, a task with a state machine is used to detect the edges in the luminance signal using the normalized signal levels. Another task with a similar state machine is used to detect the field type right after a field

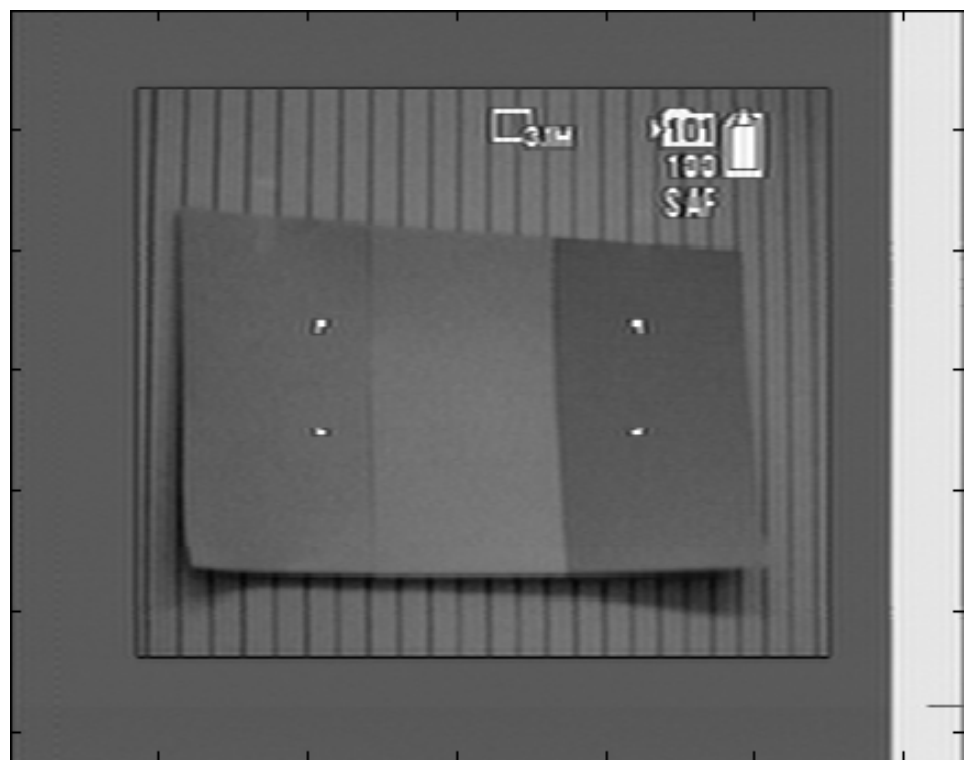

FIGURE 7.4 - Complete deinterlaced PAL video frame 


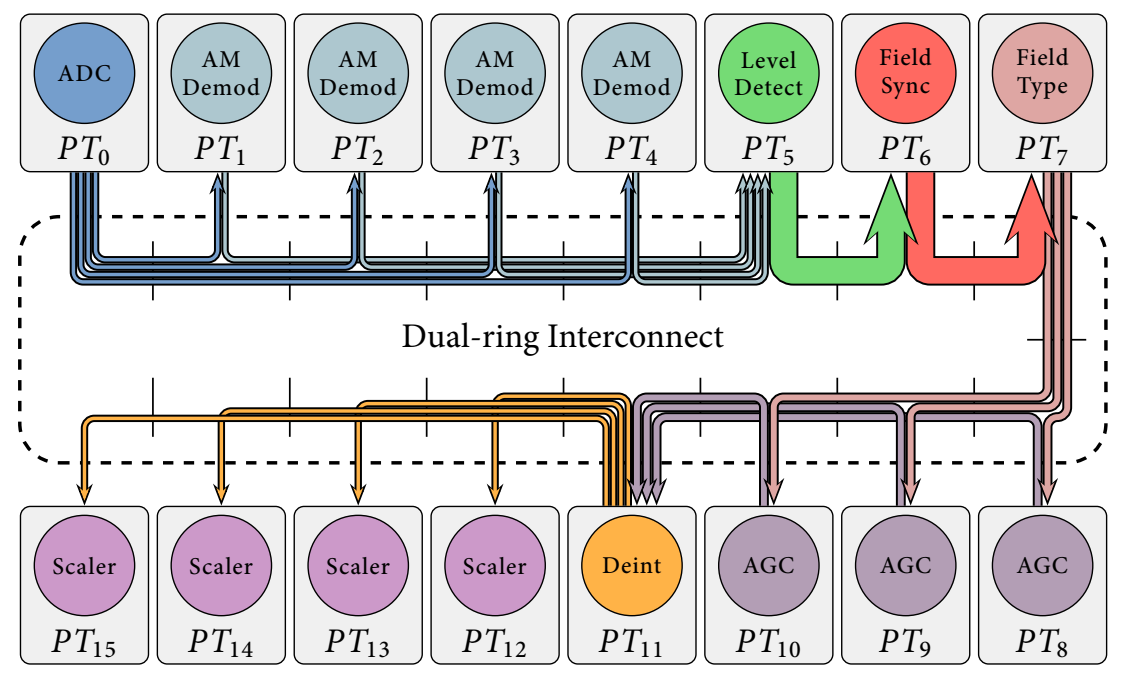

FigURE 7.5 - Mapping of our PAL video decoder onto a 16-core instance

sync was found by inspecting the signal level at the indicated positions 2 and 4 in Figure 7.1 which differs for even and odd fields. Because the two tasks contain state machines they execute sequentially and as such cannot be duplicated to benefit from task level parallelism. Other computationally bounded tasks like AM demodulation, Automatic Gain Control and Upscaling can work on small chunks of data individually and as such are duplicated to improve application throughput. The bottlenecks of the application are the field synchronization and field type detect tasks which are computationally bounded.

This algorithm uses $16 \mathrm{CPU}$ cores each running at $100 \mathrm{MHz}$ and processes PAL video real-time at $3 \mathrm{MS} / \mathrm{s}$ or $12 \mathrm{MB} / \mathrm{s}$ which corresponds to a horizontal resolution of 192 pixels. To put this in perspective, commercial grade PAL decoders require a sample rate of at least $12 \mathrm{MS} / \mathrm{s}$ which would result in a horizontal resolution of 768 pixels.

The structure of the video decoder application is written to be suitable for use with a coordination language like OIL. Because at the time Omphale did not yet support the use of stream processing accelerators, the PAL video decoder has only been used with Omphale using software tasks. As such, our demonstrators are not described in OIL but are suitable to be converted in the future when support for shared stream processing accelerators is added to Omphale.

As we use four byte wide data words and each NI transfers a complete word per clock tick, the total bandwidth between two NIs is $4 \cdot 100=400 \mathrm{MB} / \mathrm{s}$. The structure of the task graph in Figure 7.3 is similar to a processing pipeline and by using this structure as a hint for mapping to our platform, we define the mapping as shown in Figure 7.5. The width of the arrows indicating data streams denote the 
required bandwidth for that stream. The connection between two NIs is indicated by a line over the streams. Therefore, we can see from Figure 7.5 that each connection between two NIs on the data ring will transfer at most $12 \mathrm{MB} / \mathrm{s}$. This is a mere $3 \%$ of the total available bandwidth; commercial grade PAL would result in a $12 \%$ load per connection.

When we consider the longest distance data streams have to travel between tasks using the ring network, we see that data travels at most four hops down the ring before arriving at the next tile. The best case bandwidth for four hops will be $\frac{13}{16}=81.25 \%$ of $400 \mathrm{~B} / \mathrm{s}$ which is $325 \mathrm{MB} / \mathrm{s}$. Our minimum guaranteed bandwidth, regardless of the distance traveled, is $\frac{1}{16}=6.25 \%$ of $400 \mathrm{MB} / \mathrm{s}$ which is $25 \mathrm{MB} / \mathrm{s}$. This is more than the required $12 \mathrm{MB} / \mathrm{s}$ from our decoder application and therefore is sufficient.

Communication between software tasks is handled using our C-FIFO library, see Section 3.6.2, which abstracts various system aspects and presents a simple FIFO interface to the programmer. This library can also be used to perform FIFO communication using the external SDRAM memory. However, if synchronization and data are placed in external memory, we would have 16 cores contending for access while using their FIFOs. Measurements showed that the PAL decoder performs more than a factor two slower when the external memory is used instead of the data ring to transfer data.

The PAL video decoder was demonstrated at various events and is shown in Figure 7.6.

\subsubsection{Accelerated Video Decoding}

For this case study, we will improve the performance of the parallel video decoding algorithm, as shown in Figure 7.3, by replacing a computationally bounded task in software by a task implemented in hardware as an accelerator. This means the dualring interconnect is used to stream data between processor and accelerator tiles.

All four software instances of the "AM Demod" task have been replaced a single accelerator, freeing up four CPUs. The four instances of the "AM Demod" had a combined throughput of $3 \mathrm{MS} / \mathrm{s}$ or $12 \mathrm{MB} / \mathrm{s}$, where each task instance was assigned to a dedicated CPU. The fully pipelined accelerator replacing the software task has a theoretical throughput of $100 \mathrm{MS} / \mathrm{s}$ or $400 \mathrm{MB} / \mathrm{s}$. This accelerator executes the same task as the software tasks it replaces: compute the amplitude for each I/Q pair from the input data stream and convert between data formats - from fixed point to floating point. In Figure 7.2 the replacement of the tasks can be seen where all "AM Demod" tasks are replaced by the single, dashed task depicting the accelerator. The throughput of this pipelined accelerator is bounded by the speed of the "ADC" and "Level Detect" tasks, which are still implemented in software, but already improves throughput between those tasks from $3 \mathrm{MS} / \mathrm{s}$ to $11 \mathrm{MS} / \mathrm{s}$. Therefore, due to the addition of the AM decoding accelerator, the first part of the PAL video decoder can nearly operate at the speed required for commercial grade PAL decoding. 


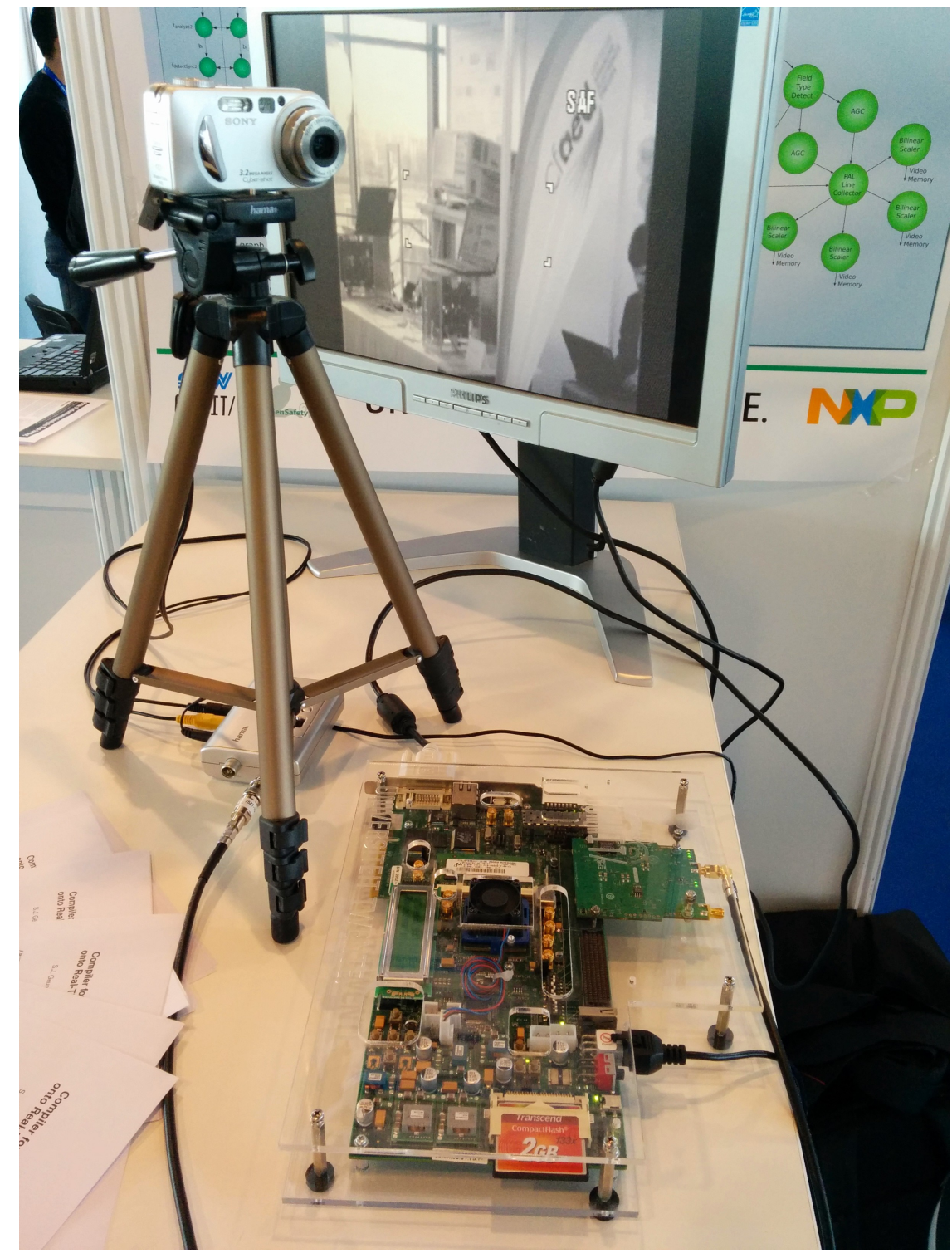

בิ

Figure 7.6 - PAL video decoder as shown on DATE 2014 


\begin{aligned} & \hline Component: Ratio: \\ & \hline Data Ring: $2.1 \% \\ &$ Credit Ring: $1.6 \% \\ &$ Shells: $4.8 \% \\ &$ AM Demod Acc.: $33 \% \\ &$\hline\end{aligned}

TABLE 7.1 - Logic usage on a Virtex 6 FPGA of the dual-ring NI components and accelerator compared to a MicroBlaze processor

The software-only video decoder requires 16 processors to obtain a $3 \mathrm{MS} / \mathrm{s}$ application throughput while the hardware accelerated video decoder requires 12 processors and one accelerator. The local throughput increase from $3 \mathrm{MS} / \mathrm{s}$ to $11 \mathrm{MS} / \mathrm{s}$ is a $366 \%$ increase due to the addition of a single accelerator which has only $\frac{1}{12}$ the hardware costs of the four processors it replaces. Considering the hardware cost from Table 7.1 (which is an extension of Table 5.2) for the dual ring, we see that the size of a dual ring NI including ring shell is roughly a quarter of the size of this small AM demodulator. Considering the throughput improvement observed and the fact that accelerators are usually bigger than a single CORDIC block, the hardware costs of the dual-ring interconnect can be attractive.

\subsubsection{Accelerated Stereo Audio Decoding}

In order to demonstrate the sharing of accelerators in our multi-core heterogeneous system under real-time constraints, an application was implemented which performs real-time decoding of a PAL stereo audio stream using two accelerators which are shared between four data streams each.

In the PAL broadcast standard, video is transmitted using an AM signal at base-

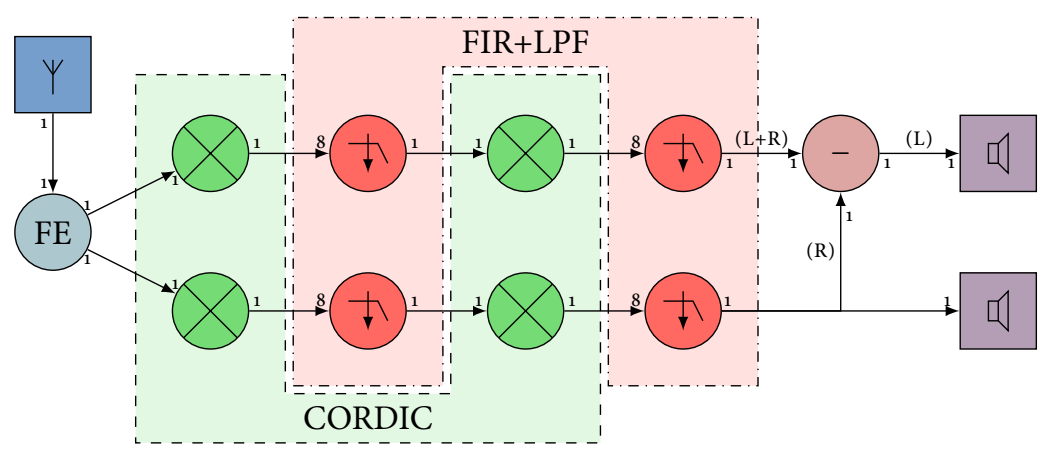

FIgURE 7.7 - Task graph of the stereo audio decoder application 
band. The audio signal is modulated at a frequency offset of $5.5 \mathrm{MHz}^{1}$ or $6 \mathrm{MHz}^{2}$ from the base frequency. The audio signal consists of one or two Frequency Modulated (FM) signals which contains mono, stereo or bilingual sound. When present, the second FM channel contains a high frequency pilot tone which denotes if the two channels form a stereo or bilingual audio signal. In this demonstrator we only support stereo audio decoding. For stereo, the first channel contains a mix of both the left and the right $(\mathrm{L}+\mathrm{R})$ channel while the second channel only contains the right audio channel (R). To obtain normal stereo the left channel (L) needs to be extracted by removing the second channel (R) from the first channel $(\mathrm{L}+\mathrm{R})$.

A schematic overview of our demonstrator is shown in Figure 7.7. In this demonstrator, the radio front-end is connected as an accelerator to our system, providing a real-time data stream on the dual-ring interconnect of the TV broadcast at baseband frequency. A channel mixer accelerator containing a CORDIC is used to mix this baseband PAL signal to the carrier frequency of one of the audio channels. A Low-Pass Filter ( $L P F)$ with built-in down-sampler is used to remove high frequency signals and obtain the FM signal. Next, an accelerator containing a CORDIC module is used to convert the data stream from FM radio to normal audio. Once again, high frequency signals are filtered out and a down-sample conversion is applied to obtain a normal sample rate for audio. This processing chain of accelerators is required for both audio channels. Reconstruction of the left channel from the $(\mathrm{L}+\mathrm{R})$ and $(\mathrm{R})$ channels is performed in a software task. The resulting two audio channels are sent to a stereo output (speakers).

In Figure 7.7 we can see that a CORDIC accelerator and a LPF+down-converter accelerator are both needed four times. Instead of duplicating this functionality, our system contains one CORDIC and one LPF+down-converter accelerator. A gateway is used to multiplex the resulting four data streams over both accelerators in real-time.

In our demonstrator, streams are switched by reading and restoring state from software. Our accelerators and exit-gateway have an execution time of one cycle/sample while the entry-gateway requires 15 cycles/sample. Reconfiguration time $\left(R_{s}\right)$ is the same for all streams and is done in 4100 cycles. Using our sharing approach, we computed that for $44.1 \mathrm{kHz}$ audio output, the streams at the start of the chain need to multiplex blocks of 10136 samples while the streams at the end of the chain will be multiplexed at 1267 samples. The $8: 1$ ratio in the block sizes is due to down-sampling.

The entry-gateway has the largest influence on throughput as it is processing data streams $5 \%$ of the time, which means that $95 \%$ of the time is spent to save and restore state from the accelerators. While we are working on techniques to improve the speed at which state can be saved and restored, our current implementation is already sufficiently fast for this application as we meet our real-time throughput constraint of $44.1 \mathrm{kS} / \mathrm{s}$ for continuous audio playback.

\footnotetext{
${ }^{1} \mathrm{PAL}-\mathrm{B} / \mathrm{G} / \mathrm{H}$

${ }^{2}$ PAL-I
} 


\begin{tabular}{rrr}
\hline Component & Slices & LUTs \\
\hline MicroBlaze CPU & 3123 & 3929 \\
Entry-gateway & 3559 & 4300 \\
Exit-gateway & 229 & 145 \\
FIR + Down-sampler & 6512 & 10837 \\
CORDIC & 1714 & 1882 \\
\hline
\end{tabular}

TABLE 7.2 - Hardware costs for the PAL stereo audio decoder

\section{Hardware Costs}

The hardware costs for components in the architecture instance are presented in Table 7.2. Our current prototype implementation of the entry-gateway is implemented using a MicroBlaze CPU. As such, the costs of the entry-gateway consist mainly of the processor and a DMA controller to stream data to the accelerators.

The exit gateway is a DMA controller which receives a data stream from the last accelerator and generates memory addresses for the processing tile which will receive the current data block of the data stream. As can be seen from the costs in Table 7.2, the exit-gateway is a small component with a small footprint.

The PAL stereo audio decoder requires a 33 taps complex FIR filter with built-in programmable down-sampler and a CORDIC block, each four times. Without accelerator sharing this application would require four instances of each accelerator type, as is depicted in the top of Figure 7.8. This would result in a lot of duplicated hardware which would also have a low utilization. In this case, by having just one of each type of accelerator, sharing implemented by the gateways removes the need for duplicate logic. The hardware costs of the gateways are much lower

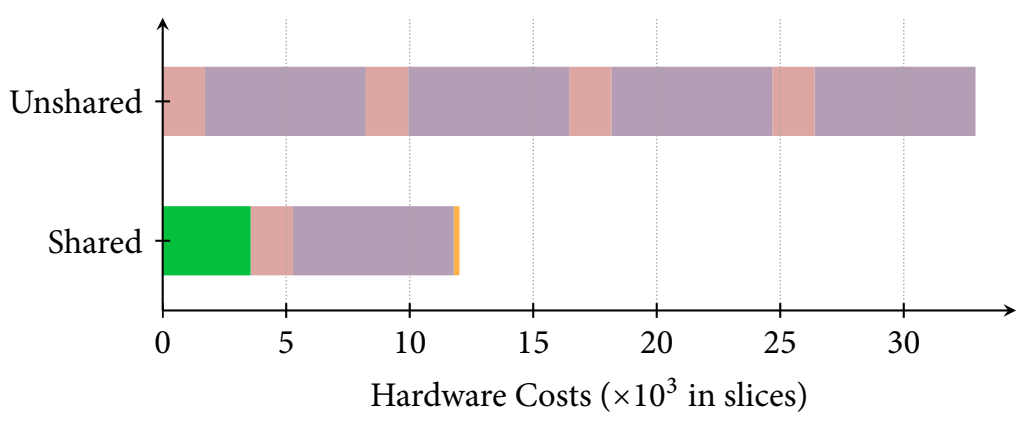

$\begin{array}{lll}\text { Entry-GW } & \text { CORDIC } \square \text { FIR }\end{array}$

FIGURE 7.8 - Costs for the PAL stereo audio decoder for the shared and non-shared approach 
than the costs of the two accelerators, resulting in a cost reduction which can be seen in Figure 7.8. Using these shared accelerators, the demonstrator still meets its throughput constraint while at the same time the hardware costs are reduced by $63 \%$ of the hardware costs compared to when accelerators are not shared.

\subsubsection{SumMary}

This case study focused on the PAL broadcast standard which due to its design allows the processing of the radio signal at a range of sample frequencies where a higher frequency corresponds with a higher quality output. This facilitates the implementation of demonstrators that can obtain real-time performance using relatively slow processors. In this section we presented three real-time demonstrators that test communication between processor tiles using the data ring, communication between processor tiles and accelerator tiles and real-time multiplexing of data streams over multiple accelerators. We evaluated the hardware costs from the respective chapters and measured the obtained performance and found that the hardware costs for the introduced dual-ring interconnect and gateways are acceptable as they improve application performance and reduce hardware costs compared to a design which does not include these components.

\subsection{GMSK DECODER}

Gaussian Minimum Shift Keying is used in various modern radio standards like GSM and Bluetooth Low-Energy and also in a new Bluetooth Long-Range proposal which is currently under discussion in a standardization committee. In this case study we will evaluate our architecture using such a Gaussian Minimum Shift Keying (GMSK) decoder application. This GMSK decoder application is implemented by a single designer using our architecture. The task graph making up this decoder is shown in Figure 7.9. In this graph we see the front-end (FE), matched filter (MF), differential quadrature demodulator (DQD), correlator (Corr), channel equalizer (Eq), error correction (FEC) and audio output (Out).

The GMSK modulation scheme is based on Minimum Shift Keying (MSK) which is a type of continuous-phase frequency-shift keying where the phase in the quadrature components denotes the bits being transmitted. A Gaussian filter is applied to suppress the sidebands in the radio spectrum which reduces out-of-band interference. The use of a Gaussian filter reduces the power density of the side lobes but causes Inter-Symbol Interference (ISI). As such the use of a channel equalizer can be beneficial.

The Viterbi Algorithm (VA) [20] is a Maximum Likelihood Sequence Estimation (MLSE) algorithm which estimates the most likely symbol values given a sequence of symbols. In our GMSK decoder, we use the VA both as a channel equalizer and as a Forward Error-Correction (FEC) decoder. When a channel equalizer outputs symbols without likelihood information, it is called hard-decision output. As the equalizer attempts to reconstruct the original signal, when using hard-decision 


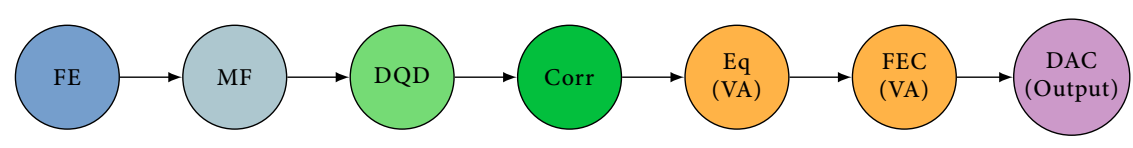

FIGURE 7.9 - Task graph of our GMSK decoder application

output it decides which symbol was most likely received. However, it does not produce an estimate of the likelihood of correctness of the produced symbols. As such, hard-decision output discards information about the probability of correctness of received symbols. The VA can consume hard-decision or soft-decision input and similarly produce both hard-decision or soft-decision output. When a convolutional decoder like Viterbi is used with a hard decision equalizer, a 1.5 to $2 \mathrm{~dB}^{3}$ sensitivity increase is observed [12]. Using an equalizer with soft-decision output with a Viterbi convolutional decoder with support for soft-decision inputs, a $4 \mathrm{~dB}^{4}$ sensitivity increase is observed [26]. This indicates that not only a channel equalizer and convolution decoder are desired to improve the sensitivity, the use of soft-decision output for such an equalizer can increase the sensitivity by another $2 \mathrm{~dB}$.

To improve the sensitivity of the next generation of radio standards, a matched filter can be used to improve the sensitivity at the cost of increasing ISI $[35,40]$. This matched filter is applied before the Differential Quadrature Demodulator (DQD) on the raw baseband signal before any other processing is applied. For acquisition, a correlator is used to search for the known pattern of the packet preamble.

The digital stream in the broadcast for our GMSK decoder is packetized where every packet is called a frame. Each frame has a known length and begins with a unique bit-sequence called a preamble. This preamble can be used by the correlator to detect the presence of a radio transmission and is used to determine moments at which the signal should be sampled.

We will explain the operations performed by the tasks in the task graph of our GMSK decoder as shown in Figure 7.9 in the following sections.

\subsubsection{Minimum Shift Keying}

Minimum Shift Keying is a special case of continuous-phase Frequency Shift Keying. In Frequency Shift Keying (FSK), the bits in the data stream are denoted by their frequency. To this end, a higher frequency can correspond to a one and a lower frequency corresponds to a zero. By using continuous-phase FSK, discontinuities in the phase are prevented which would result in very large sidebands in the frequency spectrum.

In Figure 7.10(a) it is shown that a binary data sequence is polar non-return to zero encoded. Therefore, the binary data stream is converted into a symbol sequence

\footnotetext{
${ }^{3}$ Expressed in $\frac{E_{b}}{N_{0}}, \mathrm{BT}=0.5$, taken at a BER of $10^{-3}$, code rate $r=\frac{1}{2}$

${ }^{4}$ Expressed in $\frac{E_{b}}{N_{0}}$, BT $=0.5$, taken at a BER of $10^{-3}$, code rate $r=\frac{1}{2}$
} 
where a binary 0 is mapped to a signal value of -1.0 and a 1 to signal value 1.0. In this example, the input symbol sequence is not filtered hence the in- and output are identical. In Figure 7.10(b) the phase is plotted which corresponds to the output signal in Figure 7.10(a). By determining the derivative of the phase at the correct time interval, the original symbol sequence can be reconstructed.

Continuous-phase Frequency Shift Keying works for an arbitrary pair of frequencies to denote symbols. The phase is expressed as a continuous but not always differentiable function, which can be seen in the sudden phase change when a different symbol is encoded in Figure 7.10(b). Such sudden phase changes result in large sidebands in the frequency spectrum and cause inter-channel interference.

MSK is a spectrally efficient variation of FSK where the difference between the higher and lower frequency is identical to half the symbol rate. As such, the frequencies used to denote either symbol differ by exactly half a carrier period.

Decoding FSK and MSK can be done in hardware or software where a simple comparison of the phase between two subsequent samples will determine the value of the symbol being transmitted. As it is possible that the receiver will sample at the transitions between symbol values, in our system we oversample the signal by a specific amount - currently eight times faster than the symbol rate - and use the preamble of a data frame to detect which time offset within the symbol period yields the best decoding of the signal, see also Section 7.2.3.

\subsubsection{Gaussian Minimum Shift Keying}

The Gaussian Minimum Shift Keying encoding scheme is used in radio standards like Bluetooth Low-Energy. GMSK is a variation on MSK where a Gaussian filter is used to shape the symbol sequence before being applied to a frequency modulator. This is illustrated with an example in Figure 7.11(a).

In this GMSK encoder, a symbol input stream is convoluted with a Gaussian pulseshape filter resulting in the output signal as is shown in Figure 7.11(a). The corresponding phase of the output signal is shown in Figure 7.11(b). Compared to the phase output of the MSK decoder from Figure 7.10(b), the GMSK encoder uses more gradual phase changes between symbol transitions. However, reconstructing the original symbol stream from Figure 7.11(a) is not trivial. ISI resulting from the Gaussian pulse-shape filter in the transmitter makes it difficult to distinguish between symbols. As a result it is much more difficult to retrieve the original data and channel equalization is desired.

The use of GMSK in encoding symbols results in lower sidebands and as such improves spectral efficiency. As such, channels using GMSK encoding can be transmitted on frequencies which are closer together than with FSK or MSK. 


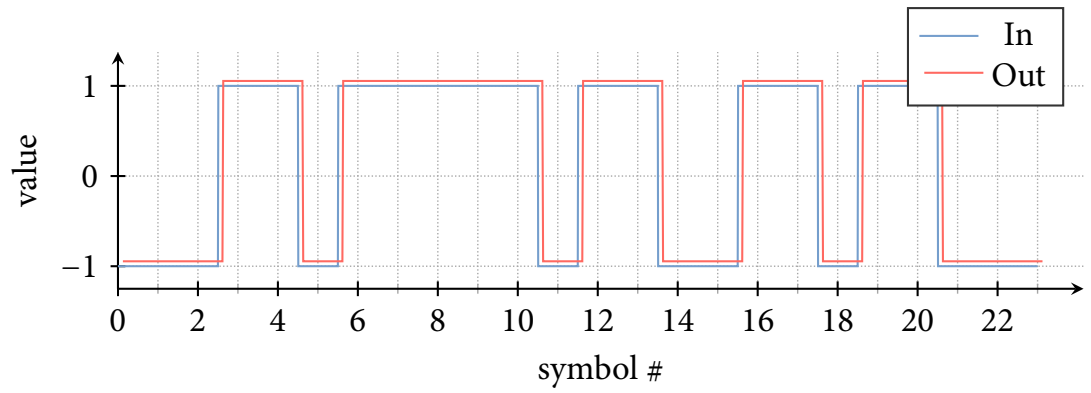

(a) Input and output signals

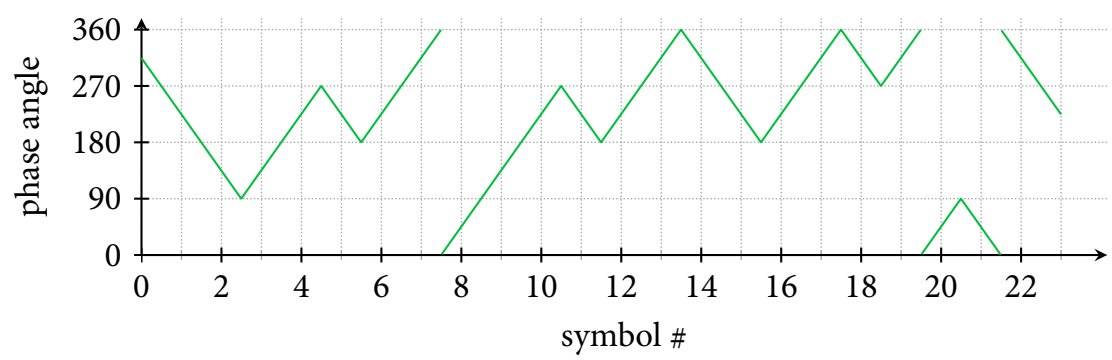

(b) Phase response of the output signal

FIGURE 7.10 - GMSK example with BT $=0.001$

\subsubsection{CORRELATOR}

Often digital data streams are divided into packets called frames. When the beginning of a frame is found, the receiver knows an estimate of the timing for every symbol to follow. In order to detect the beginning of a frame in a digitized radio signal, a correlator is used.

The correlator will detect the start of a frame by searching for a known sequence of symbols, called the preamble. As symbols can be received incorrectly, because no error-correction is applied, a sufficiently high correlation with the preamble is used to indicate the beginning of a frame. As such, the preamble should be sufficiently long that a reasonable percentage of symbols belonging to the preamble can be received in error while the detector can still detect the start of a frame. The correlator searches in an over-sampled raw data stream for the preamble using correlation and uses the best temporal offset detected in the raw data stream to lock onto the received symbols. After a temporal lock is obtained, a slicer is used to output symbols for the channel equalizer. 


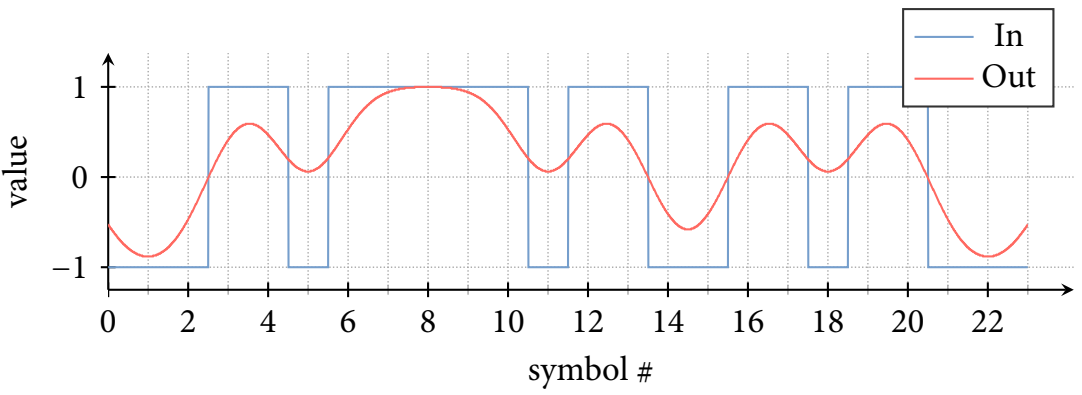

(a) Input and output signals

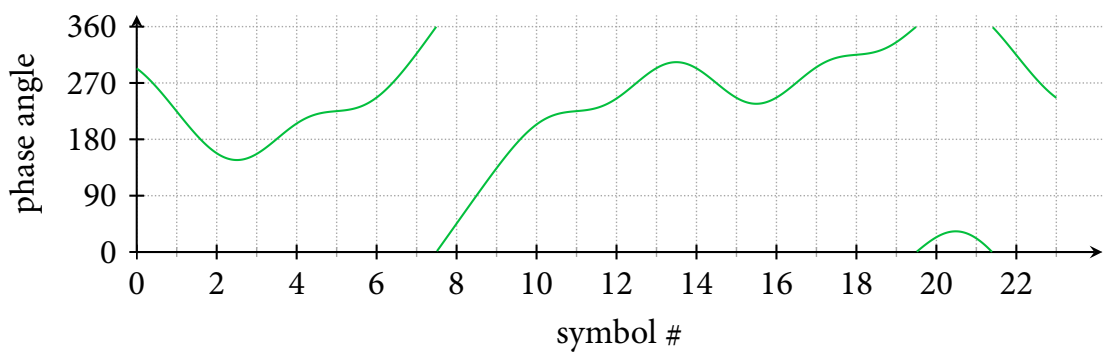

(b) Phase response of the output signal

FIGURE 7.11 - GMSK example with BT $=0.8$

In our application we used frames consisting of a preamble of 64 symbols, a sequence number of 8 bits to track lost frames, 128 bytes payload and a single tail byte. The signal is eight times oversampled, so seven in eight samples are dropped by the slicer after the preamble is detected and the best slicing point is found. Additionally, the preamble, sequence number and tail bytes are removed and only the payload is passed on.

\subsubsection{Matched Filter}

In the presence of noise, a received transmission can be severely distorted. A matched filter can be applied to detect the presence of either symbol to improve the sensitivity of the receiver. A matched filter using Laurent decomposition [48] can be used on the raw baseband signal.

Due to the matched filter, the ISI on the channel is increased. However, a channel equalizer is already required to remove ISI because of the use of a Gaussian 
pulse-shape filter at the transmitter. Therefore, the addition of a matched filter can improve the sensitivity of the receiver at only the hardware cost of the matched filter since a channel equalizer is already used.

\subsubsection{Channel Equalizer}

A channel equalizer would not only have to consider the current signal value but also the signal values of preceding and following symbols to determine the most likely transmitted symbols. This is similar to convolutional decoding which can be based on MLSE. MLSE can be done by using a VA.

By implementing a stream processing accelerator containing a Virterbi decoder which can perform both channel equalization and convolutional decoding, this accelerator can be used twice in our GMSK decoder. As the Viterbi decoder has a significant hardware cost of $65 \%$ of a single MicroBlaze core, sharing such an accelerator could yield a significant reduction in hardware cost.

\subsubsection{Forward Error Correction Decoder}

After frame synchronization and optionally channel equalization, a data stream is produced by the GMSK decoder which might contain incorrect symbols. As this can make the payload of the frame unusable, we use a form of forward error correction. This enables receiving correct symbols at lower Signal-to-Noise Ratio (SNR) levels. In our demonstrator we use the Viterbi Algorithm (VA) for convolutional decoding on the payload of the data stream, as is proposed for the Bluetooth LongRange standard.

This error correcting code works by using $n$ coding bits per $m$ bits of data (convolution), where the coding bits not only depend on the input data bit but also the previous $k$ data bits. $k$ is known as the "constraint length". The resulting code rate $r$ is $r=\frac{m}{n}$. The Viterbi decoder observes the incoming bit stream consisting of the coded data and uses a so-called Trellis diagram to compute the most likely transmitted symbols. Constraint length $k$ influences the length of the back-track used in the Trellis.

The Viterbi decoding algorithm works as follows. The Trellis begins in a known state, at the start of a frame. In convolutional coding the possibility for subsequent symbols is limited. After receiving a symbol, it updates the Trellis diagram where the likelihood that a symbol was correctly received is computed by comparing received symbols with possible transmitted symbols. The likelihood of a sequence (denoted by a path through the Trellis diagram) is derived from the Euclidean distance between the received symbols within the hard-input VA decoder and all possible transmitted symbols. Therefore, it is not necessary to retain all possible received symbol sequences - which would result in $2^{k}$ paths - but instead retain the most likely path when two options arrive at the same point in the Trellis diagram. As such, this sort of error correcting algorithms performs a maximum likelihood sequence estimation. 


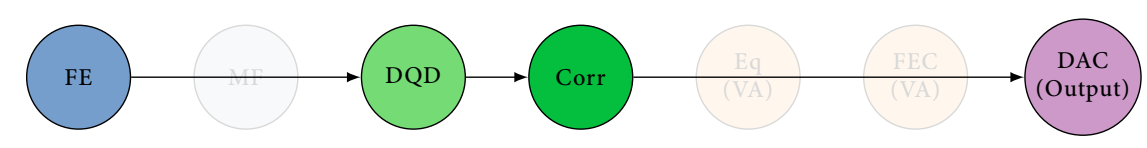

FIGURE 7.12 - Task graph of our first GMSK decoder application

\subsubsection{Audio Output}

The error corrected data stream produced by the Viterbi decoder contains a payload which depends on the radio standard. In our demonstrator, the payload consists of Pulse-Code Modulated (PCM) audio. This audio stream is converted to an analogue signal by an accelerator which produces a Pulse-Width Modulated (PWM) signal on a pin of our development board. After RC filtering, the analogue signal is sent to the speakers.

Due to the limitations of the GNUradio transmitter, the input audio is upsampled to $45.29 \mathrm{kHz}$ to obtain a baseband signal at $6.25 \mathrm{MS} / \mathrm{s}$. As a result, the sample rate at the end of the decoder chain is $45.29 \mathrm{kHz}$ as well. In order to keep the complexity of the PWM encoder low, audio samples are dropped to obtain a $44.1 \mathrm{kHz}$ output signal.

\subsubsection{Results}

We implemented three versions of our GMSK decoder: a partial implementation without matched filter and channel equalizer in software, a similar partial implementation in hardware and a complete version.

\section{Software-based Simplified GMSK Decoder}

We first implemented a simplified version of our GMSK decoder as is shown in Figure 7.12. By omitting the matched filter we reduce the ISI such that we can test the differential quadrature detector. The GMSK application from Figure 7.12 is able to decode audio in real-time but does not operate on a convolutional coded data stream.

We then implemented convolutional decoding in software using the VA. This results in the application as depicted in Figure 7.13. The software implementation of the VA was only able to process several hundred samples per second. Because realtime streaming audio requires throughput in the order of thousands samples per second, hardware acceleration is desired to increase the throughput of the convolutional decoder.

\section{Accelerated Simplified GMSK Decoder}

We implemented stream processing accelerators for the differential quadrature decoder and correlator from Figure 7.13. We additionally implemented a stream pro- 
cessing accelerator that performs the VA which can be used both as a channel equalizer and as a convolutional decoder. Our Viterbi decoder has support for soft-input to improve the decision making process during the MLSE. However, the current implementation unfortunately does not produce soft-output information.

The measured BER for our simplified GMSK decoder is shown in Figure 7.14. The correlator in Figure 7.13 can produce either hard- or soft-outputs ${ }^{5}$ for the Viterbi block. The sensitivity gain between hard-decision VA and no convolution coding is $4.5 \mathrm{~dB}$ at a BER of $10^{-3}$. However, we used an encoding rate $r$ of $\frac{m}{n}=\frac{1}{2}$ which means each data bit is encoded by two symbols and a $3 \mathrm{~dB}$ improvement in SNR is expected. When we compensate for this, we get a sensitivity gain in $\frac{E_{b}}{N_{0}}$ of $1.5 \mathrm{~dB}$.

Due to time constraints during implementation, this demonstrator uses softdecision results from a weighed mapping performed in the correlator instead of an equalizer. From Figure 7.14 we can see that the use of soft-decision input for the convolutional decoder yields another $2.5 \mathrm{~dB}$ performance gain compared to the hard-decision input. In total, the use of a soft-decision input for the convolutional decoder results in a $4 \mathrm{~dB}$ improvement to the sensitivity (in $\frac{E_{b}}{N_{0}}$ ) over the same application without the use of convolutional decoding.

Table 7.3 contains the hardware costs of the simplified implementation. We can see that the hardware costs of the Viterbi decoder is roughly $65 \%$ of the size of a complete MicroBlaze CPU.

If channel equalization would be implemented using the Viterbi decoder, we expect no reduction in hardware costs when this accelerator is shared due to the size of

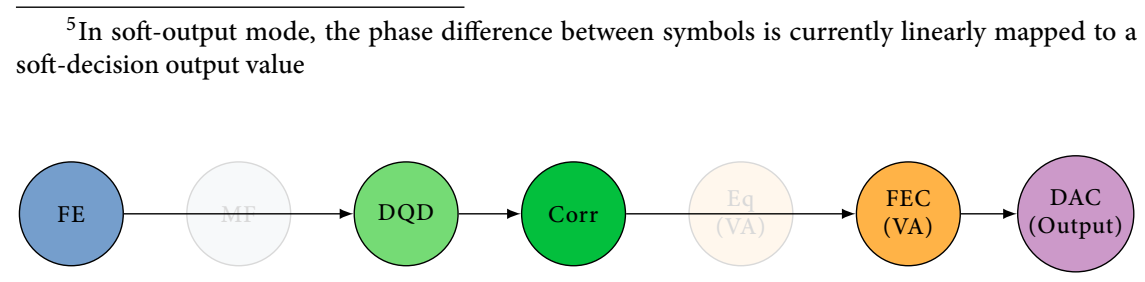

FIGURE 7.13 - Task graph of our simplified GMSK decoder application

\begin{tabular}{rrrr}
\hline & Sl. Regs & LUTs & DSP48 \\
\hline MicroBlaze CPU & 2742 & 3279 & 5 \\
Differential Quad. Demod. & 2252 & 1806 & 5 \\
Correlator & 1138 & 1760 & 0 \\
Viterbi & 969 & 2948 & 16 \\
FEC & 162 & 189 & 0 \\
DAC & 162 output & & \\
\hline
\end{tabular}

TABLE 7.3 - Hardware costs for components from the GMSK decoder 


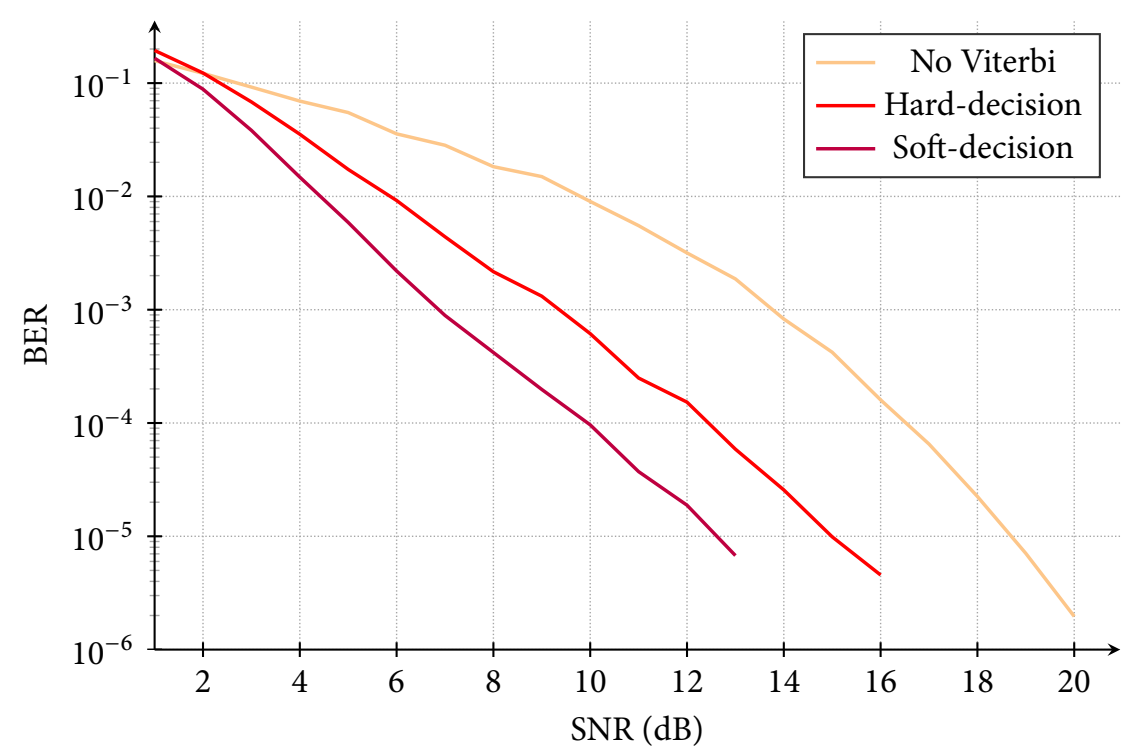

FIGURE 7.14 - Measured BER graph of our partial GMSK decoder chain $(\mathrm{BT}=0.5)$

our prototype gateways. As the current entry-gateway is based on a MicroBlaze CPU, the expected hardware costs of a shared Viterbi decoder with a gateway are $165 \%$ of a MicroBlaze CPU whereas two separate Viterbi decoders will require $130 \%$ of a MicroBlaze CPU. As such, we conclude that a reduction in hardware costs due to sharing can only be realized if the hardware costs of the gateways would be sufficiently small compared to the accelerators that will be shared.

\section{Accelerated Mode-Switching GMSK Decoder}

The accelerated GMSK decoder application from the previous section was extended with a low-pass filter, matched filter, and equalizer, as is shown in Figure 7.15. The matched filter is implemented as a FIR filter which additionally acts as a low-pass filter. Therefore, the matched filter and low-pass filter are using identical hardware.

While the matched filter and equalizer will improve the sensitivity of the received signal during the reception of a frame of data, the extra ISI introduced by the matched filter prevents the correlator to detect the preamble before a frame. Additionally, the matched filter acts as a low-pass filter resulting in a narrow band in the frequency spectrum for the equalizer to operate on while the correlator can function on a wider band in the spectrum to allow to detect frequency offsets.

To solve these issues, we implemented a GMSK channel decoder with two receiver modes, as is denoted by the dashed blocks in Figure 7.15. An accelerator implements a mode switcher and buffers enough of the data stream to seamlessly switch between detection and decoding mode. Before a frame is detected, a receiver path 


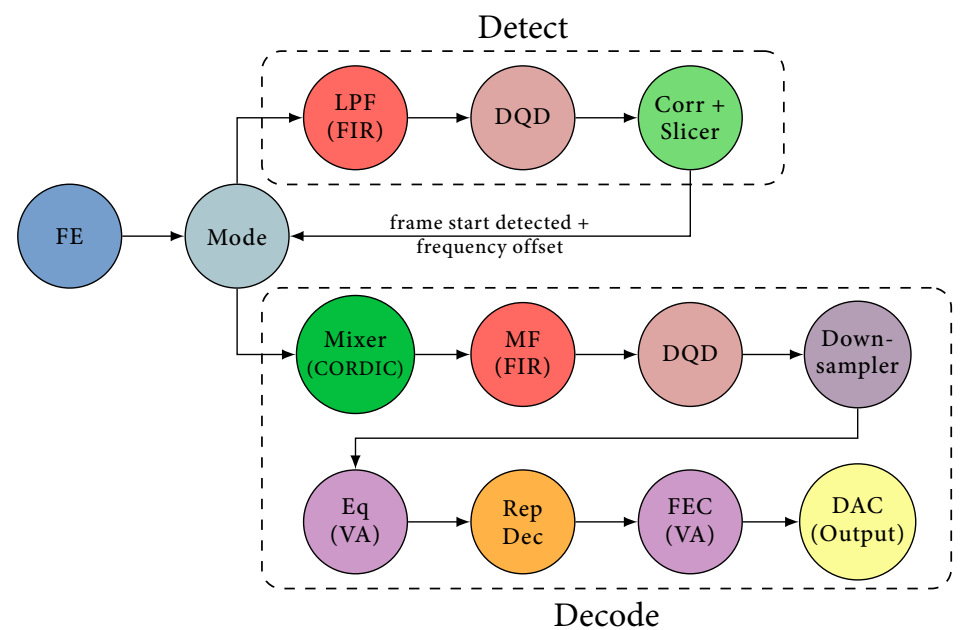

FIGURE 7.15 - Task graph of our GMSK decoder with mode switching

is used which uses a differential demodulator followed by a low-pass filter to obtain a signal with relatively low ISI. Note that while the radio receiver front-end also has a low-pass filter, the spectrum band passed on by this filter is relatively wide. As such, the addition of a low-pass filter can improve the sensitivity of the signal reception. In this signal the preamble is detected using a correlator as in the previous implementation.

When the preamble is found, information about the frequency offset and temporal offset within the signal is sent to the accelerator implementing the mode switcher. In response the mode switcher stops streaming data to the detection path and starts streaming to the decoding path. As every frame has a predefined length, the mode switcher will switch back to detection mode after all samples belonging to a single frame are passed to the decoding path. This information is then passed on to the frequency mixer in the decoding path and the down-sampler. All tasks from Figure 7.15 are implemented as accelerators aside from the down-sampler which is implemented as a software task.

Another addition to this application is the use of symbol repetition encoding. By sending out the same symbol multiple times, the repetition decoder (depicted as "Rep.Dec." in Figure 7.15) can either do slicing (hard-decision output) or compute an average over the received symbols which are supposed to be the same (softdecision output).

The hardware costs of the accelerators used in this demonstrator are shown in Table 7.4. Note how this demonstrator uses altered accelerators compared to the GMSK decoder implementations from the previous sections.

At the time of writing, this demonstrator application was completely implemented 


\begin{tabular}{|c|c|c|c|}
\hline & Sl. Regs & LUTs & $\mathrm{DSP}_{48}$ \\
\hline \multirow{5}{*}{$\begin{array}{r}\text { MicroBlaze CPU } \\
\text { Mode Switch } \\
\text { 33-taps FIR } \\
\text { (matched and low-pass filter) } \\
\text { Differential Quadrature Demod } \\
\text { Correlator + slicer }\end{array}$} & 2742 & 3279 & 5 \\
\hline & 119 & 504 & $\mathrm{o}$ \\
\hline & 1071 & 3039 & 33 \\
\hline & 2990 & 2533 & 4 \\
\hline & 191 & 675 & $\mathrm{o}$ \\
\hline$\underset{(\text { mixer })}{\text { CORDIC }}$ & 1256 & 1236 & $\mathrm{O}$ \\
\hline $\begin{array}{r}\text { Viterbi } \\
\text { (equalizer and error corrector) }\end{array}$ & 561 & 1284 & 8 \\
\hline Repetition Decoder & 325 & 298 & 1 \\
\hline $\begin{array}{r}\text { DAC } \\
\text { (PWM audio output) }\end{array}$ & 157 & 136 & $\mathrm{o}$ \\
\hline
\end{tabular}

TABLE 7.4 - Hardware costs for GMSK decoder with mode switching

and functional and preliminary measurements have been performed. The measurements of various output modes are presented in Figure 7.16.

The first few measurements are for decoding variations without mode switching. The "no ops" line denotes symbol decoding with only the quadrature differential decoder and no additional filtering or operations. The "LPF" measurement is for a decoder which uses low-pass filtering before the symbols are decoded. The "MF" measurement is for a decoder with a matched filter and a quadrature differential decoder. From these measurements we can see that the matched filter performs worse than the low-pass filter. This is to be expected as the matched filter increases the ISI.

The measurements from "LPF+MLSE" show the improvements obtained from using the VA as an channel equalizer.

The measurements denoted by "LPF+MF" are for a mode switching decoder without channel equalizer and error correction. As expected, this performs similar to the measurement with only the matched filter. However, what is not visible in this graph is that the mode switching decoder detected more frames than the decoder with only a matched filter. This is because there is no ISI from the matched filter during the preamble detection.

Finally, the "LPF+MF+MLSE" is a measurement of our mode switching GMSK decoder with a VA as channel equalizer but without error correction and repetition coding. We can see from these results that the combination of a matched filter and channel equalizer indeed results in a sensitivity improvement of roughly $2 \mathrm{~dB}$ compared to using only a channel equalizer.

We also performed measurements of our GMSK decoder with a various number of symbol repetitions. These measurements are shown in Figure 7.17. From these 


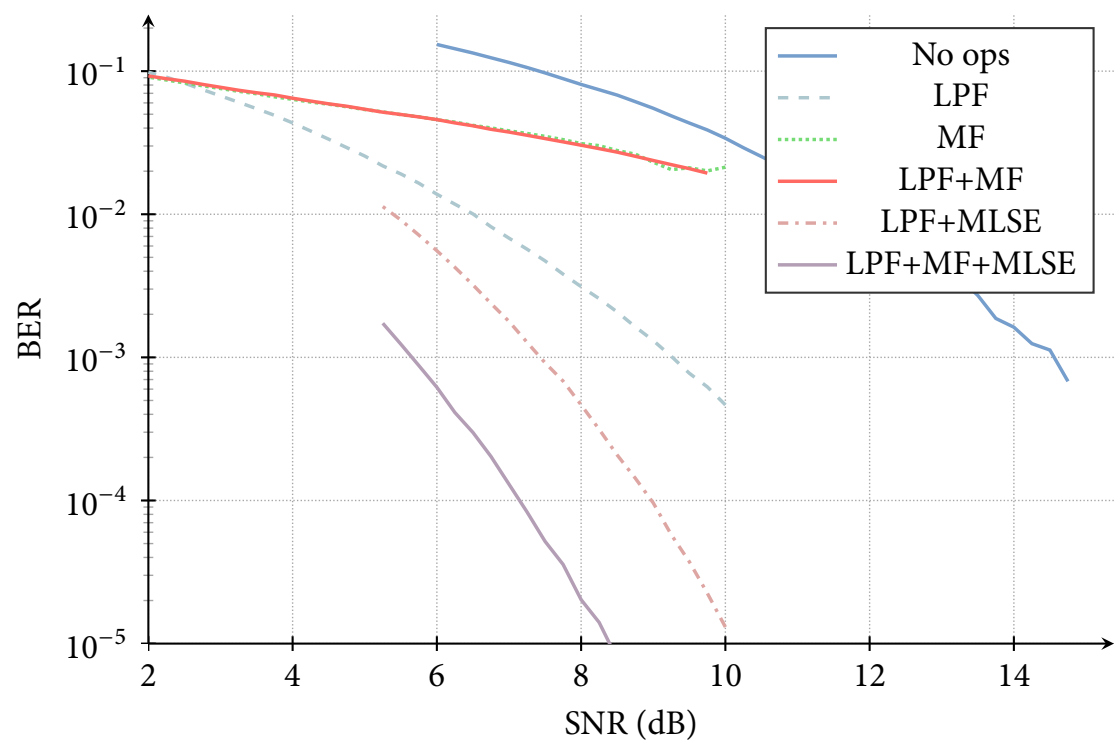

FIGURE 7.16 - Measured BER of our GMSK decoder with mode switching $(\mathrm{BT}=0.5)$

plots we can see that the repeating of symbols yields a small gain in reception when we compare single symbols to double symbol transmissions. Note that due to the double transmission the difference between single and double symbols has to be corrected for the coding gain $(-3 \mathrm{~dB})$. From these measurements it seems that repeating a symbol four times yields a worse SNR than using double repetition. The reason for this anomaly is currently being investigated.

\subsubsection{SUMMARY}

In this section, a GMSK decoder was described as a stream processing application and mapped to the proposed architecture. The resulting demonstrator decodes a GMSK encoded audio stream at 6.25 MS/s which after all decoding steps results in a real-time audio stream at $44.1 \mathrm{kHz}$.

Due to time constraints, the GMSK decoder with mode switching has been shown to be functional and the quality of reception has only been evaluated briefly. The addition of an equalizer to the realization of the GMSK decoder could be done in a more cost-effective way by duplicating the VA decoder instead of adding gateways to share the existing VA decoder. However, since an instance of our architecture was used with a single VA accelerator which can be shared, we conclude that an implementation with two physical VA accelerators is not required to add a VA equalizer to the application. This also means that if the design of the architecture was fixed, for example because it was implemented as an Application-Specific Integrated Circuit (ASIC), support for sharing of accelerators would facilitate this 


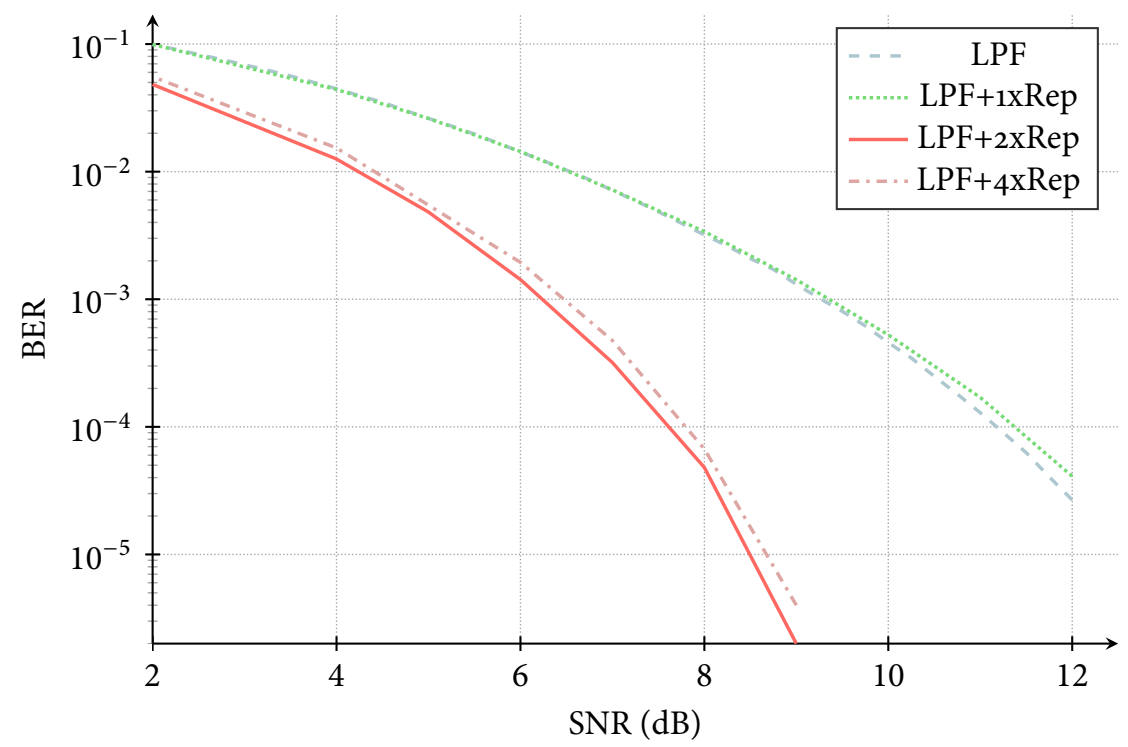

FIGURE 7.17 - Measured BER graph of our GMSK decoder with repetition coding $(\mathrm{BT}=0.5)$

change to the application without requiring alterations to the hardware.

We implemented the Viterbi Algorithm (VA) in a stream processing accelerator and performed measurements of the obtained Bit Error Rate (BER). The hardware costs of the VA accelerator were less than a single MicroBlaze while its throughput is several orders of magnitude higher than a software implementation. The evaluation of the obtained gains in sensitivity from the use of the complete GMSK decoder application with mode switching was not finished at the time of writing. We conclude that without stream processing accelerators this demonstrator can not be executed in real-time on our architecture.

\subsection{CONCLUSions}

The experiments and their results performed during these case studies demonstrate the functionality of software and hardware tasks and accelerator multiplexing in order to improve utilization. For the first case study, a multi-core PAL video decoder application has been implemented in software for a 16 core system using only the data ring where communication is handled by our C-FIFO implementation. In a second experiment a variation on the software PAL video decoder is implemented where one of the computationally bound tasks was replaced by an accelerator. This modification frees up four processors and improves throughput by $366 \%$ compared to the software-only case, demonstrating the merit of using accelerators for such tasks. The description of the PAL video decoder as a task graph allows to trivially 
replace a software task by a stream processing accelerator.

In the second case study, an application is implemented which performs stereo FM audio demodulation using two shared accelerators that each process four data streams. The results from this experiment demonstrate that stream processing accelerators can be shared under real-time constraints when our sharing approach is implemented by the gateways. The current prototype has a relatively high overhead when switching between data streams but already suffices for the considered applications. Because the hardware costs of the shared stream processing accelerators is higher than the addition of the gateways, a reduction in hardware costs is realized.

Results in the third case study demonstrate that the integration of stream processing accelerators facilitates the design and testing of more complex radio receivers than would be possible using a homogeneous architecture. In this case study a GMSK decoder was found to be too slow for real-time stream processing using software decoding tasks. The addition of a number of accelerators to perform the same computationally intensive tasks enabled real-time stream processing for a single audio channel. Sharing of accelerators facilitates the evaluation of multiple variations of the GMSK radio decoder where for example a single VA accelerator is used not only for convolutional decoding but also for channel equalization.

We observe that due to the size of the prototype of our gateways sharing a single stream processor accelerator which implements the VA does not yield a reduction hardware costs compared to duplication of the VA accelerator. However, as explained in Section 6.5.1, we expect that a significant reduction in hardware costs can be attained when our sharing approach is implemented in hardware. We conclude that this case study demonstrates that a reduction in hardware costs due to sharing can only be realized if the hardware costs of the gateways would be sufficiently small compared to the accelerators that will be shared.

It is shown that hardware costs can be reduced and throughput can be increased by introducing hardware accelerators without the need for significant changes of the application software. Furthermore, the structured design of our presented architecture results in a system complexity which is manageable by a single designer. This is illustrated by the fact that the GMSK decoder application was build by a single designer using our proposed architecture. These case studies demonstrate that the proposed real-time heterogeneous multi-core stream processing architecture is suitable for the considered stream processing applications. 


\section{Conclusion}

In this thesis a new architecture was introduced for real-time stream processing applications. This architecture is better suited for real-time stream processing applications than the SMP and the NUMA architectures which contains aspects from both. Support for integration of shared stream processing accelerators improves computation capacity over a homogeneous design at a lower hardware cost while retaining the programming flexibility usually associated with homogeneous multicore architectures.

Functionality implemented in stream processing accelerators is often computationally intensive which would result in a low throughput on a general purpose processor. Examples shown in this thesis are CORDIC algorithms or FIR filters. Such functionality is often required multiple times in one application. At the same time, the accelerators are often not fully utilized by a single data stream, wasting processing capacity. It is therefore desired to share stream processing accelerators between data streams.

The proposed architecture described in this thesis is designed for real-time applications. Real-time implies that data entering the system has to be processed before a certain deadline expires.

In order for an architecture to be suitable for real-time applications, for each component or composition of components a predictable worst-case bound should be obtainable. In our architecture we use mostly components for which a worst-case bound on latency and throughput can be obtained in isolation. An example of such a component for which worst-case bounds can be obtained is the communication infrastructure. This means that for such components a temporal model should be available which allows efficient analysis to derive sufficiently tight bounds on the worst-case temporal behavior. In our architecture, both the dual-ring interconnect and gateways used for accelerator sharing allow the derivation of real-time guarantees.

Stream processing applications can be described as a graph of tasks that use FIFO channels to communicate. Therefore, communication and computation is separate. 
While the communication between tasks can be described in a coordination language, the actual implementation of tasks can be done in other software languages or by using hardware stream processing accelerators.

Communication between each pair of tasks can be seen as point-to-point streaming in a heterogeneous MPSoC. To this end, an interconnect is required that can facilitate master-slave access to memories and at the same time provide point-topoint data streaming. These requirements can be satisfied by various existing interconnects but those often come at a high hardware cost compared to the network proposed in this thesis.

The low-cost dual-ring interconnect proposed in this thesis has support for guaranteed throughput and point-to-point data streaming. Our ring interconnect has a low hardware cost due to the fact that it is connectionless: we do not need separate buffers in the NI for every connection. At the same time, our arbitration policy provides real-time guarantees for all communication. As such, we presented a connectionless network with guaranteed throughput. Without support for connections or virtual channels, there is no overhead - in cycles or in hardware costs - to set up or tear down connections. Our design imposes no limit on the number of concurrent communications on the ring interconnect. As such, our ring interconnect can be seen as mix of a traditional bus and a NoC.

A small shell in the NI provides support for stream processing accelerator integration. As a credit counter is required for every connection to an accelerator, communication involving accelerators currently only supports a single connection at a time per processor to keep hardware costs low.

We propose a sharing approach which facilitates sharing of one or more accelerators between data streams under real-time constraints. To this end, our approach only allows a stream to be considered for processing by the accelerators if the buffer at the output of the last accelerator in a chain has space to store a complete block of data to prevent stalls for space. Additionally, context switching of accelerators is only allowed after all data belonging to a block of data has been processed. This approach is modeled in a CSDF model to facilitate independent temporal analysis between streams. We implemented our sharing approach using so-called gateways.

A number of case studies have been carried out to evaluate the dual-ring interconnect, accelerator integration and sharing of accelerators. In the first case study we used a PAL video decoder application to evaluate real-time point-to-point streaming and accelerator integration. In the second case study we used a PAL stereo audio decoder application to evaluate real-time stream processing, accelerator integration and sharing of accelerators. The last case study is focused on the implementation of a real-time GMSK decoder application. This application did not meet the real-time requirements on our system when implemented using only software tasks. As a result, it required the use of stream processing accelerators of which some could be shared.

In the remainder of this chapter we will first recapitulate the results of the previous 
chapters. We will then present our contributions in detail in Section 8.2. We will end this chapter with suggestions for future work.

\subsection{ReCApitulation}

In this thesis we presented an architecture for real-time stream processing applications. Stream processing applications can be described as a graph of communicating tasks, where each task operates on a data stream of potentially infinite length. SDR applications are examples of stream processing applications. Most SDR applications have real-time requirements: if the stream processing is not fast enough, data will be corrupted.

In Chapter 1 we introduced a classification for multi-core architectures. These classes were the SMP class, which is usually a homogeneous multi-core design, and the NUMA class which is often a heterogeneous design. The shared central memory used in multi-core SMP architectures make them often easy to program but also results in poor scalability in the number of cores. The use of multiple memories in the NUMA class reduces the contention for memory access at the cost of increased programming efforts. In this work we introduced an architecture which is optimized for real-time stream processing applications. In this architecture we support the classical master-slave protocol for the exchange of data in memories. At the same time we support a protocol for point-to-point data streaming for communication with stream processing accelerators. In this architecture, each PE has its own local memory (with read/write access) and data is streamed to local memories of other cores when needed (write-only). A central memory is used to store program instructions and large data structures, for example complete video frames.

In Chapter 2 we presented related work concerning stream processing architectures. We concluded that only a few architectures are designed for real-time applications and only the CompSOC architecture is providing real-time guarantees by means of temporal analysis techniques similar to our work. Often interconnects with support for guaranteed throughput have high hardware costs, such as the Æthereal NoC (or one of its varieties) which is also used in CompSOC. Those high hardware costs are partly due to the support for virtual channels. Another aspect leading to high hardware costs is the requirement for dedicated buffers in every NI per connection. Our dual-ring interconnect does not support virtual channels, reduces hardware costs by sharing buffers at the NIs and at the same time supports guaranteed throughput. Mesh-shaped interconnects and crossbars are proposed to replace traditional single- or multi-layer buses but are not often used in practice as their hardware costs are considerable. Lately ring-shaped topologies have been used as an alternative to traditional buses as they tend to have very low hardware costs and can often provide enough bandwidth to be an attractive solution.

In Chapter 3 we introduced our heterogeneous multi-core architecture for realtime stream processing applications. Processing elements are grouped into tiles which are interconnected by two networks. Our dual-ring interconnect provides 
real-time point-to-point data streaming, i.e. communication which is not masterslave based and with flow control. A secondary tree-shaped interconnect provides master-slave based access to shared components such as the SDRAM. The architecture is built using four tile types: processor tiles, accelerator tiles, entry- and exit-gateway tiles. Processor tiles use a FIFO scheme for communication which is implemented in software using the C-HEAP protocol. Writing into a full FIFO and reading from an empty FIFO is not possible. Accelerator tiles make use of a credit-based flow control scheme which is provided by the shells in the NIs.

Software tasks communicate using our unidirectional communication ring. This ring interconnect realizes low hardware costs by sharing of buffers within the NIs as is described in Chapter 4. This is possible due to the automatic serialization of the ring, which prevents head-of-line blocking and requires guaranteed acceptance at the slaves which is provided by memories. Despite being connectionless, our ring provides bandwidth and latency guarantees by reserving slots for each master. The work-conserving scheduling policy of the ring interconnect allows tasks to use unused slots of other tasks. We implemented a software-based FIFO using the write-only network by making use of a split FIFO administration. We derived an SDF model for the communication channel between two software tasks which can be used to determine the required FIFO capacity given the throughput constraints of the application. Implementations for FPGA and ASIC confirmed that the ring scales linearly with the number of cores and occupies only $2 \%$ of the hardware resources used in the architecture.

An additional unidirectional ring is used when accelerators need to be integrated as presented in Chapter 5. This secondary ring is used for the hardware implementation of a credit-based flow control scheme. The credit ring is optimized to transfer credits - which are a single bit wide - and does not transfer data. As a result, the hardware costs of the credit ring are lower than those of the data ring. We derived a CSDF model which describes a FIFO between two tiles using the credit-based flow control scheme. This model can be used to determine the required FIFO capacity in order to reduce the effects of network latency on application throughput. We found that our dual-ring interconnect occupies $8.5 \%$ of the resources in a 16 core instance of our architecture when implemented on a Virtex 6 FPGA.

In Chapter 6 we presented an approach for sharing stream processing accelerators under real-time constraints. This approach is implemented using entry- and exitgateways. Sharing reduces the need for duplicated functionality and improves utilization of accelerators. We presented a CSDF model which can be abstracted into a model which consists of a single SDF node to model a gateway and a chain of accelerators which are shared between multiple streams. This model can be used to check if throughput constraints are satisfied when block sizes are specified. An ILP is presented to compute minimum block sizes under a throughput constraint. However, we showed that minimizing the block sizes reduces the required buffer capacities but does not necessarily result in the minimal buffer capacities due to the non-monotonic relation between block sizes and buffer capacities given a through- 
put constraint. Furthermore, we concluded that sharing can not only result in a reduction in hardware costs but also improves the flexibility after a design is implemented in silicon.

The case studies are presented in Chapter 7. These case studies have been carried out to demonstrate that the complexity of the architecture remained manageable by a single designer and to evaluate the performance and hardware cost of the architecture instances. From the first case study we concluded that a computationally bounded software task can be replaced by a stream processing accelerator in our architecture with only minor changes to the top-level software description of the application. This modification frees up four processors and improves throughput by $366 \%$ compared to the software-only case. During the second case study, we implemented an application which performs stereo audio demodulation using two shared accelerators that each process four data streams. With this design we demonstrated that stream processing accelerators can be shared under real-time constraints using our sharing approach with gateways. As a third case study we implemented a GMSK decoder application. From this case study we conclude that the integration of stream processing accelerators is necessary for performance reasons as an homogeneous architecture could not provide the required throughput. Accelerator sharing facilitates the use of a Viterbi accelerator in the GMSK decoder application for both channel equalization and forward error correction. We observed that due to the current hardware costs of an entry-gateway - mostly due to the size of the used MicroBlaze processor - a reduction in hardware costs can only be attained if enough and sufficiently large accelerators are shared compared to the case where accelerators are simply duplicated.

\subsection{Contributions}

We reiterate the main contributions of this thesis:

1. Introduction of a real-time heterogeneous stream processing architecture that allows efficient compositional performance analysis and supports both masterslave and streaming communication protocols such that stream processing accelerators and a shared memory is supported.

In this thesis we introduced a new architecture which has novel features for stream processing like point-to-point streaming support and at the same time borrows some aspects from SMP and NUMA architectures, keeping programming effort acceptable. The introduced dual-ring interconnect and gateways are designed in such a way that real-time guarantees can be provided for those components locally. Previously introduced components [66] also have real-time guarantees provided, such as the tree-shaped interconnect used for master-slave communication with components such as the shared central memory. The guarantees derived from components can be used in an application-level model used for efficient compositional performance analysis for an application. From this analysis we can derive 
real-time bounds for applications being executed on the proposed hardware architecture.

2. Introduction of a predictable low-cost communication interconnect with a ring-shaped topology.

Our single communication ring does not require support for back-pressure in the network and allows sharing of buffers in the NIs resulting in a simple and low-cost design. This single ring interconnect can be used by our software FIFO implementation to provide point-to-point data streaming with back-pressure between software tasks under real-time constraints (Chapter 4).

3. The creation of a temporal analysis model for this interconnect in the form of an SDF model.

The single ring uses a slot reservation mechanism such that an SDF model can be constructed which models the properties of the ring and the software FIFO implementation. This temporal analysis model can be used to determine the required FIFO capacity in order to satisfy the application-level throughput constraint (Chapter 4).

4. The creation of hardware flow control by introducing a second ring for oppositely directed flow control packets. This facilitates the integration of hardware stream processing accelerators that can not be used to implement flow control in software.

Stream processing accelerators do not support a master-slave protocol and thus can not communicate directly with memories. Therefore, a small shell in each NI provides a streaming interface for stream processing accelerators. This shell implements credit-based flow control and writes data using key-hole addresses to keep hardware costs low. Processors using hardware flow control can read or write one word which can result in a processor stall when the buffer is full. In this fashion there is no overhead for the processor to check for space or data but at the same time only a single task can be executed on a processor when real-time guarantees are to be provided (Chapter 5).

5. The creation of a temporal analysis model of the dual-ring interconnect in the form of a CSDF model.

The properties of the two rings used to construct the dual-ring interconnect are used in a CSDF model to accurately describe communication with stream processing accelerators (Chapter 5). This CSDF model is introduced as it was shown that the less accurate SDF model from Chapter 4 would incorrectly - indicate that communication involving accelerators causes the system to deadlock.

6. The development of an approach to share stream processing accelerators by different data streams under real-time constraints.

Round-robin time sharing of stream processing accelerators between multiple data streams uses context-switches of the internal state of the accelerators and reconfiguration of the stream processing accelerators. These 
context switches can only be correctly done when all data sent to the accelerators has been completely processed. This ensures that no data is en route in an interconnect to or from an accelerator, or that data from one data stream ends up in an accelerator configuration context of another data stream as this would yield corrupt results. Our sharing approach uses entryand exit-gateways to determine that a complete block of data can be stored at the output of the last accelerator. When all data sent to one or more accelerators has been processed the accelerators can be switched to the next data stream (Chapter 6).

7. The creation of an SDF model which enables the computation of the granularity at which blocks of data must be multiplexed over stream processing accelerators given real-time constraints. Computation of block sizes is a novel application of SDF analysis.

A CSDF model is constructed to describe the sharing of stream processing accelerators. This model is abstracted into an SDF model which simplifies temporal analysis at the application level. The granularity at which data streams are multiplexed is expressed in the data block size for each multiplexed stream. From the temporal analysis model we can see the trade-off where smaller block sizes can result in lower latency but higher overhead resulting from the context-switch between streams. The firing duration of the actor denoting the shared accelerator is depending on the block sizes of each stream using that accelerator. This results in a mutual dependency when determining the minimum required block sizes to obtain the required throughput. We propose the use of an ILP to obtain a feasible solution under the constraint that each actor denoting a shared accelerator is not part of any cycle in the dataflow model besides the cycle introduced for modeling back-pressure (Chapter 6).

8. The implementation and evaluation of instantiations of the proposed architecture on a Virtex 6 FPGA. For evaluation purposes a PAL video decoder has been developed given the specification of the PAL broadcast standard. Additionally, a stereo FM audio decoder and a GMSK decoder have been implemented.

The dual-ring interconnect and gateways are implemented and tested in multiple instances of our architecture. The hardware costs and application performance were presented. From the results we concluded that stream processing accelerator integration can improve throughput considerably compared to a homogeneous design without a substantial increase in hardware costs of the communication networks. Sharing of accelerators can reduce hardware costs but the introduced gateway prototypes are relatively expensive compared to the accelerators used in our applications. Sharing of accelerators provides flexibility for applications where new applications can be mapped on existing hardware when functionality provided by accelerators is required more than once (Chapter 7). 


\subsection{Future Work}

Our dual-ring interconnect is designed to provide guaranteed throughput, is workconserving and has a low hardware cost compared to a NoC with support for virtual channels. Our sharing approach improves flexibility of an architecture implementation and can reduce hardware costs. From our evaluation we have seen that the architecture presented in this thesis is well suited for the considered real-time stream processing applications.

We see a number of options to be explored further:

\section{Cost-Efficient Accelerator Integration}

Results from the case studies indicate that the integration of stream processing accelerators is implemented in a cost-effective way. Therefore, small accelerators can be introduced for operations that are performed on a data stream. For example the introduction of an accelerator performing an addition on elements of a data stream would result in an accelerator with a relatively large overhead in the costs for the NI. However, the cost compared to a MicroBlaze processor is low and the expected throughput of such an accelerator is expected to be an order of magnitude higher compared to a MicroBlaze. This raises the interesting question what the granularity of the accelerators should be in order to be beneficial and thus considered for inclusion in the proposed architecture.

\section{Better Bandwidth Control}

Currently, our implementation and models use a certain fixed bandwidth distribution which allows us to provide real-time guarantees. An interesting option is to explore methods for better bandwidth control per application to improve the performance.

Methods that would improve control over bandwidth are for example:

" The characteristics of the average case throughput and latency can be adjusted without affecting the worst-case throughput and latency by reordering slots on the ring. As such, it can be desirable to make the slot ordering configurable to allow various optimizations depending on the application.

» Techniques like masking can be used to prevent NIs to use specific slots, guaranteeing their availability to other NIs.

" Unused PEs on the ring could be forced to yield their slot to be available to all others on the ring. This could be implemented with small changes to the arbitration policy and can improve for example the bandwidth for communications traveling many hops.

» To redistribute bandwidth, slot numbers could be assigned multiple times. 


\section{Reduce Power Consumption}

An area we did not explore in this thesis is the reduction of power consumption for various components of the dual-ring interconnect. Our current design realizes low hardware costs which indirectly results in a relatively low power budget. However, we observed in Section 4.7 that in the general case the power consumption could be reduced by ordering the slots according to Gray coding or reduce the average latency for applications with a lot of non consecutive writes (not burst-writes) such as can be found in the SDR domain.

Another option to explore is the clock gating of components that are not active during specific cycles. For example, in the NIs the data and address registers can be clock gated for empty slots - e.g. slots without valid data. NIs that are not in use during (part of) the execution of an application can be mostly clock gated to reduce power.

\section{Hierarchical Rings}

Our current architecture employs a dual-ring interconnect which provides connectivity to all PEs. During experiments with various SDR applications we observed that functionality provided by accelerators is often used in a specific order and in a certain part of an application. As such, a hierarchical ring interconnect might improve scalability of our architecture by clustering functionality on smaller rings while higher level rings provide intra-cluster communication. It would be very interesting to see if a hierarchical network can improve latency and throughput in such a manner that real-time guarantees can be given.

\section{Scheduling Policy for Streams}

The order in which streams are considered for multiplexing on shared accelerators is done in round-robin fashion in our architecture. An interesting topic would be to evaluate which other scheduling policies can be beneficial for use in a real-time stream processing architecture. These results might differ significantly depending on the application, indicating that this is a question with a non-trivial answer. Changing the scheduling policy for multiplexing would potentially require alterations to the SDF model that was introduced in Chapter 6.

\section{Mapping}

Assignment of tasks to PEs is currently done by hand. The only available mapping algorithm employed by Omphale attempts to perform load-balancing over all available cores based on the computational load of every task. This algorithm does not take properties of the underlying interconnect into account. Additionally, determining which tasks are suitable for data-level parallelism and thus duplication to improve throughput is an open problem. 
While the Omphale compiler supports mapping of software tasks to processor tiles, at the time of writing there is no support for shared stream processing accelerators ${ }^{1}$. In this case the mapping should be done manually which is error prone.

Another aspect to consider during mapping is the expected application performance as a result of worst-case throughput and latency for communication between tasks. The placement of tasks on the PEs in the design will greatly influence the obtained throughput.

We therefore see the interesting problem that we lack an algorithm which attempts to load-balance the tasks over all PEs while at the same time considers the properties of the underlying interconnect. Using knowledge of data streams in the interconnect allows the derivation of tighter worst-case bounds and should use techniques for better bandwidth control, as was suggested in Section 8.3.

\section{Entry-Gateway Improvements}

The current entry-gateway is implemented using a MicroBlaze processor and a DMA controller. However, our sharing approach does not require a software implementation of the sharing approach. We expect that a hardware-only implementation of the entry-gateway can be implemented with a hardware footprint which is much smaller than the current implementation.

Currently a new method of reconfiguring accelerators is being implemented which removes the need for a separate configuration bus for each accelerator. Instead, configuration and state for accelerators is loaded and stored using the dual-ring interconnect and reconfiguration of accelerators is done in a streaming fashion. To this end, the configuration and internal state for accelerators is injected in the data stream sent to the stream processing accelerators. As a result, the cycle after an accelerator completes processing of a block of data, the reconfiguration of that accelerator is performed. This means it is no longer necessary to wait for the accelerators to complete the processing of a block of data before reconfiguration can be sent, reducing the time required to switch between data streams even further.

Additionally, such an implementation should have a lower overhead in the time required to switch between data streams reducing the minimum block sizes required for multiplexing data streams in order to obtain the same throughput compared to the current entry-gateway implementation. This will further reduce the sizes of the buffers holding the data blocks, reducing hardware costs even more.

\section{Real-time Behavior Of Caches}

Not for all components in our architecture hard real-time guarantees can be provided. The use of caches in our processors results in good performance but due to the use of these caches no tight real-time guarantees can be given for reads and

\footnotetext{
${ }^{1}$ There is preliminary support for the integration of accelerators
} 
writes to shared memory. As such, it is interesting to look into approaches to obtain such guarantees for safety-critical programs.

One approach is to prohibit the direct access to shared memory altogether and use DMA controllers to write data to shared memory explicitly by the programmer [28]. In this manner, programs are executed using local memories and writes to remote memories are handled by the DMA controllers, both with a fixed latency resulting in predictable behavior. The downside of this approach is that programs can not be executed when their size exceeds the available local memory. Therefore, it is interesting to explore the use of software managed SPMs [5]. This is similar to a software-managed cache where required memory segments are loaded on-demand in a predictable manner. Ideally, such an approach would be implemented in a way that is transparent to the programmer. 


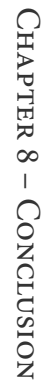




\section{ACronyms}

\begin{tabular}{|c|c|c|}
\hline A & ALU & Arithmetic Logic Unit \\
\hline & $\mathrm{AM}$ & Amplitude Modulated \\
\hline & ASIC & Application-Specific Integrated Circuit \\
\hline B & $\mathrm{BE}$ & Best Effort \\
\hline & BER & Bit Error Rate \\
\hline $\mathrm{C}$ & $\mathrm{CM}$ & Cycle Mean \\
\hline & CSDF & Cyclo-Static Data Flow \\
\hline D & DMA & Direct Memory Access \\
\hline & DQD & Differential Quadrature Demodulator \\
\hline & DSP & Digital Signal Processing \\
\hline $\mathrm{F}$ & FCFS & First-Come-First-Served \\
\hline & FEC & Forward Error-Correction \\
\hline & FIR & Finite Impulse Response \\
\hline & FM & Frequency Modulated \\
\hline & FSK & Frequency Shift Keying \\
\hline & FSL & Fast Simplex Link \\
\hline G & GMSK & Gaussian Minimum Shift Keying \\
\hline & GPU & Graphical Processing Unit \\
\hline & GT & Guaranteed Throughput \\
\hline $\mathrm{H}$ & HSDF & Homogeneous Synchronous Data Flow \\
\hline I & ILP & Integer Linear Program \\
\hline & ISE & Instruction-Set Extension \\
\hline & ISI & Inter-Symbol Interference \\
\hline $\mathrm{L}$ & LPF & Low-Pass Filter \\
\hline 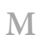 & MCM & Maximum Cycle Mean \\
\hline & MLSE & Maximum Likelihood Sequence Estimation \\
\hline & MPSoC & Multiple Processor System on Chip \\
\hline & MSK & Minimum Shift Keying \\
\hline $\mathrm{N}$ & NI & Network Interface \\
\hline & NoC & Network on Chip \\
\hline & NUMA & Non-Uniform Memory Architecture \\
\hline & OS & Operating System \\
\hline
\end{tabular}




\begin{tabular}{|c|c|c|}
\hline \multirow{5}{*}{134} & PAL & Phase Alternating Line \\
\hline & PCM & Pulse-Code Modulated \\
\hline & $\mathrm{PE}$ & Processing Element \\
\hline & PT & Processor Tile \\
\hline & PWM & Pulse-Width Modulated \\
\hline \multirow{10}{*}{$\begin{array}{l}\text { D } \\
0 \\
0 \\
z \\
z \\
z \\
\infty\end{array}$} & RPC & Remote Procedure Call \\
\hline & $\mathrm{RR}$ & Round-Robin \\
\hline & RTDMA & Rotating Time Division Multiplex Access \\
\hline & SDF & Synchronous Data Flow \\
\hline & SDR & Software Defined Radio \\
\hline & SIMD & Single Instruction Multiple Data \\
\hline & SIMT & Single Instruction Multiple Threads \\
\hline & SMP & Symmetric Multi Processor \\
\hline & SNR & Signal-to-Noise Ratio \\
\hline & SPM & Scratchpad Memory \\
\hline $\mathrm{T}$ & TDM & Time Division Multiplexing \\
\hline $\mathrm{V}$ & VA & Viterbi Algorithm \\
\hline W & WCET & Worst Case Execution Time \\
\hline
\end{tabular}




\section{List of Symbols}

$V \quad$ Set of all actors within a dataflow model.

$B_{c} \quad$ Bandwidth of a communication channel $c$, expressed as a fraction.

$\omega_{s} \quad$ Combined waiting duration of all other streams than $s$ over a gateway.

$\hat{\omega}_{s} \quad$ Worst case upper bound of $\omega_{s}$.

$\tau_{b} \quad$ Duration of processing a single block of $\eta$ samples when multiplexing.

$\hat{\tau}_{b} \quad$ Worst case upper bound of $\tau_{b}$.

$\eta \quad$ Block sized used for multiplexing data stream over an accelerator.

$\gamma_{s} \quad$ Total duration for one cycle of a shared accelerator processing one block for every stream.

$\hat{\gamma}_{s} \quad$ Worst case upper bound of $\gamma_{s}$.

$\psi \quad$ Depth of the FIFO at the input of a FIFO.

$D$ Size of a data container in words as used in a C-FIFO channel.

$\varphi_{s} \quad$ Worst case time spent writing a container of $S$ words belonging to stream $s$ to the ring.

$\varphi \quad$ Worst case time spent writing a container of $S$ words to the ring.

$c_{x} \quad$ Denotes a cycle $x$ in a dataflow graph.

$n_{x} \quad$ Number of tokens on the cycle $c_{x}$.

$C_{G} \quad$ Denotes all cycles belonging to dataflow graph $G$.

$e_{a, b} \quad$ Edge from $v_{a}$ to $v_{b}$.

$v \quad$ Actor in a dataflow model.

E Set of all edges between actors within a dataflow model. 


\section{List OF Figures}

$1.1 \quad$ Utility vs time . . . . . . . . . . . . . . . . . . . . . . . 5

1.2 Example of the SMP architecture class . . . . . . . . . . . . . . 7

1.3 NUMA class ... . . . . . . . . . . . . . . . . . . 8

1.4 Schematic overview of master-slave communication ........ . . 9

1.5 Schematic overview of point-to-point communication . . . . . . 10

1.6 SPA class . . . . . . . . . . . . . . . . . . . . . . . 12

2.1 An example of a co-processor ... . . . . . . . . . . . . 23

2.2 An example of a stream processing accelerator . . . . . . . . . . 24

3.1 Example architecture overview with all types of tiles . . . . . . . 33

3.2 Processor Tile . . . . . . . . . . . . . . . . . . . . . . 35

3.3 Accelerator Tile . . . . . . . . . . . . . . . . . . . . . . . . . . 37

3.4 Entry-gateway . . . . . . . . . . . . . . . . . . . . . . 38

3.5 Exit-gateway . . . . . . . . . . . . . . . . . . . . . . . . . . . . . 39

3.6 Single ring design consisting of chained ring Network Interfaces . . . 40

3.10 Examples for a distributed FIFO with capacity $3 \ldots . . . . . . .43$

4.1 Ring slot arbitration example for a word write . . . . . . . . . 52

4.2 Schematic overview of a single NI . . . . . . . . . . . . . 53

4.3 SDF graph for a task with core-to-core communication . . . . . . . 54

5.1 Schematic overview of a single NI. * Accelerators only read and write data and omit addresses. . . . . . . . . . . . . . . . 66

5.2 Potentially dead-locked model for accelerator communication . . . 68

5.3 CSDF model of hardware FIFO communication . . . . . . . . . . 68

5.4 HSDF model of a Latency-Rate Server . . . . . . . . . . . . . 70

5.5 CSDF model of hardware FIFO including channel characteristics . . 71

5.6 HSDF obtained from transformation of the CSDF model from Fig-

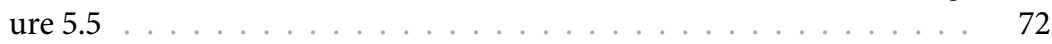

6.1 Basic idea for sharing accelerators . . . . . . . . . . . . . . . 79

6.2 CSDF model of accelerator multiplexing for a data stream $s \ldots . . .81$

6.3 Execution schedule of stream $s$ processing blocks of $\eta_{s}$ samples . . . 83

6.4 SDF abstraction for a data stream $s$ over an accelerator $v_{s} \ldots \ldots 83$

6.5 SDF model . . . . . . . . . . . . . . . . . . . . . . . . . . . 85

6.6 Task graph showing shared FIFO sharing . . . . . . . . . . 86 
6.7 Hardware costs of various components in a Virtex 6 FPGA . . . . 87

6.8 Example of an hierarchical ring with sub-rings grouping functionality 89

7.1 ITU-R 470-6 PAL timing diagram for field synchronization . . . . . 96

7.2 Complete PAL line with two low valued synchronization pulses . . . 97

7.3 Task graph of our PAL decoder application . . . . . . . . . . . . . . 98

7.4 Complete deinterlaced PAL video frame . . . . . . . . . . . . . . 98

7.5 Mapping of our PAL video decoder onto a 16-core instance . . . . . 99

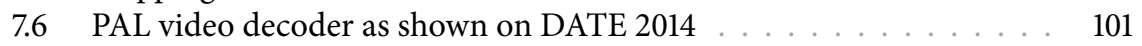

7.7 Task graph of the stereo audio decoder application . . . . . . . . . 102

7.8 Costs for the PAL stereo audio decoder for the shared and non-shared

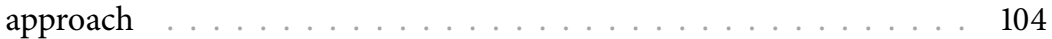

7.9 Task graph of our GMSK decoder application . . . . . . . . . . 106

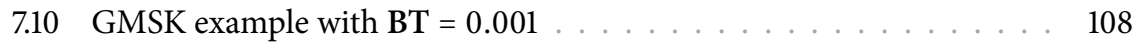

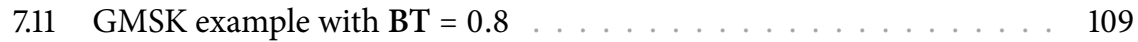

7.12 Task graph of our first GMSK decoder application . . . . . . . . . . . 111

7.13 Task graph of our simplified GMSK decoder application . . . . . . . 112

7.14 Measured BER graph of our partial GMSK decoder chain $($ BT = 0.5) . 113

7.15 Task graph of our GMSK decoder with mode switching . . . . . . . . 114

7.16 Measured BER of our GMSK decoder with mode switching (BT = 0.5) 116

7.17 Measured BER graph of our GMSK decoder with repetition coding $(\mathrm{BT}=0.5)$ 


\section{LIST OF TABLES}

1.1 Comparison of types of programming languages

1.2 Architecture classification overview (SPA = Stream Processing Architectures)

2.1 Comparison of architectures

2.2 Comparison of Networks-on-Chip (NoCs)

4.1 Logic usage on a Virtex-6 FPGA showing a linear scale in resource usage for our ring interconnect.

4.2 Post-synthesis results of $65 \mathrm{~nm}$ ST implementation in ASIC logic, leakage is specified after power.

5.1 MCM for various $D$ and $\alpha$ on for hardware FIFO communication between a processor and accelerator.

5.2 Logic usage on a Virtex 6 FPGA of the dual-ring NI components compared to a MicroBlaze processor

6.1 Buffer capacities for various $\eta_{s}$

6.2 Hardware costs and savings for a demonstrator implemented in a Virtex 6 FPGA

7.1 Logic usage on a Virtex 6 FPGA of the dual-ring NI components and accelerator compared to a MicroBlaze processor

7.2 Hardware costs for the PAL stereo audio decoder

7.3 Hardware costs for components from the GMSK decoder

7.4 Hardware costs for GMSK decoder with mode switching 


\section{BibLIOGRAPHY}

[1] Advanced Micro Devices. 3DNow! Technology Manual. 2010. URL http:// support . amd. com/TechDocs/21928.pdf. (Cited on page 26).

[2] Altera Corperation. Nios II Custom Instruction User Guide. 2011. (Cited on page 26).

[3] Altera Corporation. Avalon Interface Specifications. May 2013. (Cited on page 24).

[4] ARM. AMBA AXI and ACE Protocol Specification. 2011. (Cited on page 22).

[5] O. Avissar, R. Barua, and D. Stewart. An optimal memory allocation scheme for scratch-pad-based embedded systems. ACM Transactions on Embedded Computing Systems (TECS), 1(1):6-26, Nov. 2002. ISSN 1539-9087. doi: 10.1145/581888.581891. (Cited on page 131).

[6] C. Bartels, J. Huisken, K. Goossens, P. Groeneveld, and J. Meerbergen. Comparison of An Æthereal Network on Chip and A Traditional Interconnect for A MultiProcessor DVB-T System on Chip. In Proc. Int'l Conference on Very Large Scale Integration (VLSI-SoC), pages 80-85, New York, NY, USA, Oct. 2006. IEEE. ISBN 3-901882-19-7. doi: 10.1109/VLSISOC.2006.313208. (Cited on page 27).

[7] G. Bilsen, M. Engels, R. Lauwereins, and J. Peperstraete. Cyclo-static dataflow. IEEE Transactions on Signal Processing, 44(2):397-408, 1996. ISSN 1053587X. doi: 10.1109/78.485935. (Cited on pages 64, 67, 71, 72, 78, and 81).

[8] S. Blagodurov, S. Zhuravlev, M. Dashti, and A. Fedorova. A Case for NUMA-aware Contention Management on Multicore Systems. In USENIX Annual Technical Conference (USENIX ATC), pages 1-15, New York, NY, USA, 2011. ACM Press. (Cited on page 8$)$.

[9] F. Conti, A. Marongiu, and L. Benini. Synthesis-friendly techniques for tightlycoupled integration of hardware accelerators into shared-memory multi-core clusters. In Proc. Int'l Conf. on Hardware/software codesign and system synthesis (CODES+ISSS), pages 1-10, New York, NY, USA, Sept. 2013. IEEE. ISBN 978-1-47991417-3. doi: 10.1109/CODES-ISSS.2013.6658992. (Cited on page 25).

[10] A. Cox and R. Fowler. The implementation of a coherent memory abstraction on a numa multiprocessor: Experiences with platinum. In Proc. ACM Symposium on Operating Systems Principles (SOSP), pages 32-44, New York, NY, USA, 1989. ACM Press. ISBN 0-89791-338-8. doi: 10.1145/74850.74855. (Cited on page 8).

[11] A. Dasdan and R. Gupta. Faster maximum and minimum mean cycle algorithms for system-performance analysis. IEEE Transactions on Computer-Aided Design of Integrated Circuits and Systems, 17(10):889-899, 1998. ISSN 02780070. doi: 10.1109/43.728912. (Cited on page 55). 
[12] R. D’Avella, L. Moreno, and E. Turco. Adaptive equalization and viterbi decoding for digital mobile radio systems. In Proc. Global Telecommunications Conference, pages 90-94, New York, NY, USA, Nov. 1989. IEEE. doi: 10.1109/GLOCOM.1989.63946. (Cited on page 106).

[13] B. D. de Dinechin. . In Dataflow language compilation for a single chip massively parallel processor, page 1, New York, NY, USA, Sept. 2013. IEEE. doi: 10.1109/MuCoCoS.2013.6633597. (Cited on page 20).

[14] B. D. de Dinechin. Kalray mppa: Manycore challenges for the next generation of professional applications, July 2013 (accessed Januari 22, 2015). URL http: //www . mpsoc-forum.org/previous/2013/slides/12-De_Dinechin.pdf. Conference slides. (Cited on page 20).

[15] R. de Groote, J. Kuper, H. Broersma, and G. J. Smit. Max-Plus Algebraic Throughput Analysis of Synchronous Dataflow Graphs. In Proc. Euromicro Conference on Software Engineering and Advanced Applications, pages 29-38, New York, NY, USA, Sept. 2012. IEEE. ISBN 978-0-7695-4790-9. doi: 10.1109/SEAA.2012.20. (Cited on page 55).

[16] developerWorks. Meet the experts: David krolak on the cell broadband engine eib bus, Dec. 2005 (accessed Januari 22, 2015). URL http://www.ibm.com/ developerworks/power/library/pa-expert9/. (Cited on page 20).

[17] EPIQ Solutions. Bitshark FMC-1RX Rev C User's Manual. 2011. (Cited on page 94).

[18] C. Fallin, X. Yu, G. Nazario, and O. Mutlu. A high-performance hierarchical ring onchip interconnect with low-cost routers. Technical Report SAFARI Technical Report No. 2011-007, Computer Architecture Lab (CALCM), Carnegie Mellon University, 2011. (Cited on pages 21, 23, and 51).

[19] M. J. Flynn, O. Pell, and O. Mencer. Dataflow supercomputing. In Proc. Int'l Conf. on Field Programmable Logic and Applications (FPL), pages 1-3, New York, NY, USA, Aug. 2012. IEEE. ISBN 978-1-4673-2256-0. doi: 10.1109/FPL.2012.6339170. (Cited on page 25).

[20] G. D. Forney Jr. The viterbi algorithm. Proceedings of the IEEE, 61(3):268-278, 1973. doi: 10.1109/PROC.1973.9030. (Cited on page 105).

[21] O. P. Gangwal, A. Nieuwland, and P. Lippens. A scalable and flexible data synchronization scheme for embedded HW-SW shared-memory systems. In Int'l Symposium on System Synthesis (ISSS), pages 1-6, New York, NY, USA, 2001. ACM Press. ISBN 1581134185. doi: 10.1145/500002.500003. (Cited on pages 27 and 36).

[22] M. Geilen, T. Basten, and S. Stuijk. Minimising buffer requirements of synchronous dataflow graphs with model checking. In Proc. Design Automation Conference (DAC), pages 819-824, New York, NY, USA, 2005. ACM Press. (Cited on pages 84 and 85$)$.

[23] S. J. Geuns, J. P. H. M. Hausmans, and M. J. G. Bekooij. Sequential Specification of Time-aware Stream Processing Applications. ACM Transactions on Embedded Computing Systems (TECS), 12(1s):35:1-35:19, Mar. 2013. ISSN 1539-9087. doi: 10.1145/2435227.2435231. (Cited on page 3). 
[24] S. J. Geuns, J. P. Hausmans, and M. J. Bekooij. Hierarchical programming language for modal multi-rate real-time stream processing applications. In Proc. Int'l Conf. on Parallel Processing Workshops (ICPPW), pages 453-460, Washington, D.C., USA, Sept. 2014. IEEE Computer Society. doi: 10.1109/ICPP.Workshops.2014.66. (Cited on page 20 ).

[25] K. Goossens and A. Hansson. The Aethereal network on chip after ten years: Goals, evolution, lessons, and future. In Proc. Design Automation Conference (DAC), pages 306-311, 2010. ISBN 9781450300025. (Cited on pages 21, 22, 25, and 59).

[26] J. Hagenauer and P. Hoeher. A viterbi algorithm with soft-decision outputs and its applications. In Proc. Global Telecommunications Conference, pages 1680-1686, New York, NY, USA, Nov. 1989. IEEE. doi: 10.1109/GLOCOM.1989.64230. (Cited on pages 94 and 106).

[27] A. Hansson. A Composable and Predictable On-Chip Interconnect. $\mathrm{PhD}$ thesis, Technical University of Eindhoven, June 2009. (Cited on pages 8 and 19).

[28] A. Hansson, K. Goossens, M. Bekooij, and J. Huisken. CoMPSoC: A template for composable and predictable multi-processor system on chips. ACM Transactions on Design Automation of Electronic Systems (TODAES), 14(1):1-24, 2009. ISSN 10844309. doi: 10.1145/1455229.1455231. (Cited on pages 18 and 131).

[29] A. Hansson, M. Wiggers, A. Moonen, K. Goossens, and M. J. G. Bekooij. Enabling application-level performance guarantees in network-based systems on chip by applying dataflow analysis. IET Computers \& Digital Techniques, 3(5):398, 2009. ISSN 17518601. doi: 10.1049/iet-cdt.2008.0093. (Cited on page 54).

[30] M. Harmanci, N. Escudero, Y. Leblebici, and P. Ienne. Providing QoS to connectionless packet-switched NoC by implementing diffserv functionalities. In Proc. Int'l Symposium on Systems on Chip (SoC), pages 37-40, New York, NY, USA, 2004. IEEE. ISBN 0-7803-8558-6. doi: 10.1109/ISSOC.2004.1411140. (Cited on page 16).

[31] J.P. H. M. Hausmans. Abstractions for aperiodic multiprocessor scheduling of real-time stream processing applications. PhD thesis, University of Twente, Apr. 2015. (Cited on page 80 ).

[32] J. P. H. M. Hausmans, S. J. Geuns, M. H. Wiggers, and M. J. G. Bekooij. Temporal analysis flow based on an enabling rate characterization for multi-rate applications executed on mpsocs with non-starvation-free schedulers. In Proc. Int'l Workshop on Software and Compilers for Embedded Systems (SCOPES), pages 108-117, New York, NY, USA, 2014. ACM Press. ISBN 978-1-4503-2941-5. doi: 10.1145/2609248.2609262. (Cited on page 69).

[33] C. Helmstetter, S. Basset, R. Lemaire, F. Clermidy, P. Vivet, M. Langevin, C. Pilkington, P. Paulin, and D. Fuin. A dynamic stream link for efficient data flow control in NoC based heterogeneous MPSoC. In Proc. Design Automation Conference. Asia and South Pacific (ASP-DAC), pages 41-46, New York, NY, USA, Jan. 2013. IEEE. doi: 10.1109/ASPDAC.2013.6509556. (Cited on pages 18 and 19). 
[34] L. J. Hendren, J. Hummel, and A. Nicolau. Abstractions for Recursive Pointer Data Structures: Improving the Analysis and Transformation of Imperative Programs. In Proc. Conf. on Special Interest Group on Programming Language Design and Implementation (SIGPLAN), pages 249-260, New York, NY, USA, June 1992. ACM Press. ISBN 0-89791-475-9. doi: 10.1145/143095.143138. (Cited on page 2).

[35] K. Hwang and M. Wickert. A soft output GMSK demodulator using a 4-filter MLSE for small BT product. In Proc. Int'l Conf. on Communications (ICC), volume 5, pages 2957-2961, New York, NY, USA, 2002. IEEE. doi: 10.1109/ICC.2002.997382. (Cited on page 106).

[36] IBM. 128-Bit Processor Local Bus Architecture Specifications - Version 4.7. 2007. (Cited on page 22).

[37] Intel. Intrinsics for intel streaming simd extensions, Oct. 2014. URL software. intel. com/en-us/node/514302. (Cited on page 26).

[38] T. International Business Machines Corporation, Sony Computer Entertainment Inc. Cell Broadband Engine Programming Handbook: Including the PowerXCell 8i Processor (version 1.12). Apr. 2009. (Cited on page 20).

[39] International Telecommunication Union. Rec. ITU-R BT.470-6: Conventional Television Systems. Technical report, 1998. (Cited on page 95).

[40] G. Kaleh. Simple coherent receivers for partial response continuous phase modulation. IEEE Selected Areas in Communications, 7(9):1427-1436, Dec. 1989. ISSN 0733-8716. doi: 10.1109/49.44586. (Cited on page 106).

[41] Kalray. Mppa manycore. Brochure, 2014. URL http://www. kalrayinc .com/IMG/ pdf/FLYER_MPPA_MANYCORE.pdf. (Cited on page 8).

[42] N. Kavaldjiev, G. Smit, P. Jansen, and P. Wolkotte. A virtual channel network-onchip for GT and BE traffic. In Proc. Symposium on VLSI, page 6, Mar. 2006. doi: 10.1109/ISVLSI.2006.13. (Cited on pages 17 and 18).

[43] B. Khailany, W. Dally, U. Kapasi, P. Mattson, J. Namkoong, J. Owens, B. Towles, A. Chang, and S. Rixner. Imagine: media processing with streams. IEEE Micro, 21(2):35-46, 2001. ISSN 02721732. doi: 10.1109/40.918001. (Cited on page 24).

[44] M. Kistler, M. Perrone, and F. Petrini. Cell Multiprocessor Communication Network: Built for Speed. IEEE Micro, 26(3):10-23, May 2006. ISSN 0272-1732. doi: 10.1109/MM.2006.49. (Cited on pages 21, 23, and 25).

[45] A. G. C. Koppelaar, A. Burchard, and W. Tang. Concurrent Viterbi decoding for dual-channel ITS communication on a SDR platform. In 33rd WIC Symposium on Information Theory in the Benelux, pages 149-156, 2012. (Cited on pages 21 and 22).

[46] S. Kottapalli and J. Baxter. Nehalem-EX CPU Architecture. Hot chips, pages 1-19, 2009. (Cited on pages 21, 23, and 25).

[47] N. Kung and R. Morris. Credit-based flow control for ATM networks. IEEE Network, 9(2):40-48, 1995. ISSN 08908044. doi: 10.1109/65.372658. (Cited on pages 42 and 65). 
[48] P. Laurent. Exact and Approximate Construction of Digital Phase Modulations by Superposition of Amplitude Modulated Pulses (AMP). IEEE Transactions on Communications, 34(2):150-160, 1986. ISSN 0090-6778. doi: 10.1109/TCOM.1986.1096504. (Cited on page 109).

[49] E. Lee and D. Messerschmitt. Synchronous data flow. Proceedings of the IEEE, 75(9): 1235-1245, 1987. ISSN 0018-9219. doi: 10.1109/PROC.1987.13876. (Cited on page 56).

[50] J. A. J. Leijten and J. L. van Meerbergen. Stream communication between real-time tasks in a high-performance multiprocessor. In Proc. Design, Automation and Test in Europe Conference and Exhibition (DATE), pages 125-131, 1998. ISBN 0-8186-8359-7. doi: 10.1109/DATE.1998.655846. (Cited on page 27).

[51] F. Lemonnier, P. Millet, G. M. Almeida, M. Hubner, J. Becker, S. Pillement, O. Sentieys, M. Koedam, S. Sinha, K. Goossens, C. Piguet, M.-N. Morgan, and R. Lemaire. Towards future adaptive multiprocessor systems-on-chip: An innovative approach for flexible architectures. In Proc. Int'l Conf. on Embedded Computer Systems (SAMOS), pages 228-235. IEEE, July 2012. ISBN 978-1-4673-2297-3. doi: 10.1109/SAMOS.2012.6404179. (Cited on pages 18, 19, and 26).

[52] J. H. Lienhard. A heat transfer textbook. Courier Dover Publications, 2011. ISBN 978-0486479316. (Cited on page 2).

[53] D. Liu, D. Wiklund, and E. Svensson. SoCBUS: The solution of high communication bandwidth on chip and short TTM. In Proc. Real-Time and Embedded Computing Conference (RTECC), 2002. (Cited on pages 17, 21, 22, and 25).

[54] A. Molnos, J. A. Ambrose, A. Nelson, R. Stefan, S. Cotofana, and K. Goossens. A composable, energy-managed, real-time MPSOC platform. In Proc. Int'l Conf. on Optimisation of Electrical and Electronic Equipment (OPTIM), pages 870-876, New York, NY, USA, 2010. IEEE. ISBN 978-1-4244-7019-8. doi: 10.1109/OPTIM.2010.5510478. (Cited on page 18).

[55] O. Moreira, J. Mol, M. Bekooij, and J. van Meerbergen. Multiprocessor Resource Allocation for Hard-Real-Time Streaming with a Dynamic Job-Mix. In Proc. IEEE RealTime and Embedded Technology and Applications Symposium (RTAS), pages 332-341, New York, NY, USA, 2005. IEEE. ISBN 0-7695-2302-1. doi: 10.1109/RTAS.2005.33. (Cited on page 83).

[56] A. Nieuwland and J. Kang. C-HEAP: A heterogeneous multi-processor architecture template and scalable and flexible protocol for the design of embedded signal processing systems. Design Automation for Embedded Systems, 7(3):233-270, 2002. (Cited on pages 25 and 41).

[57] OpenMP Architecture Review Board. The openmp api specification for parallel programming, 1997 (accessed Januari 15, 2015). URL http://openmp . org/. (Cited on page 2).

[58] M. Panić, G. Rodriguez, E. Quiñones, J. Abella, and F. J. Cazorla. On-chip ring network designs for hard-real time systems. In Proc. Int'l Conference on Real-Time Networks and Systems, page 23, New York, NY, USA, 2013. ACM Press. ISBN 978-14503-2058-0. doi: 10.1145/2516821.2516829. (Cited on page 25). 
[59] R. Pellizzoni, B. Bui, M. Caccamo, and L. Sha. Coscheduling of CPU and I/O Transactions in COTS-Based Embedded Systems. In Proc. Real-Time Systems Symposium, pages 221-231, New York, NY, USA, Nov. 2008. IEEE. ISBN 978-0-7695-3477-0. doi: 10.1109/RTSS.2008.42. (Cited on page 7).

[60] R. Pellizzoni, A. Schranzhofer, J.-J. Chen, M. Caccamo, and L. Thiele. Worst case delay analysis for memory interference in multicore systems. In Proc. Design, Automation and Test in Europe Conference and Exhibition (DATE), pages 741-746, New York, NY, USA, Mar. 2010. IEEE. ISBN 978-1-4244-7054-9. doi: 10.1109/DATE.2010.5456952. (Cited on page 7).

[61] É. Piel, P. Marquet, J. Soula, and J.-L. Dekeyser. Asymmetric Scheduling and Load Balancing for Real-Time on Linux SMP. In Parallel processing and applied mathematics, volume 3911 of Lecture Notes in Computer Science, pages 896-903. Springer Berlin Heidelberg, 2006. ISBN 978-3-540-34141-3. doi: 10.1007/11752578_108. (Cited on page 7).

[62] F. J. Pollack. New microarchitecture challenges in the coming generations of cmos process technologies (keynote). In Proc. ACM/IEEE Int'l Symposium on Microarchitecture (MICRO), page 2, Washington, D.C., USA, 1999. IEEE Computer Society. ISBN 0-7695-0437-X. (Cited on page 2).

[63] Recore Systems. Many-core processor subsystem ip-on-fpga, Nov. 2014 (accessed Januari 23, 2015). URL http://www.recoresystems.com/fileadmin/ downloads/Product_briefs/2014-02_MCP_SIP_Product_brief_1.0.pdf. Product Brief. (Cited on page 8).

[64] A. Rege. An introduction to modern gpu architecture, Dec. 2008 (accessed Januari 15, 2015). URL ftp: //download.nvidia. com/developer/cuda/seminar/TDCI_ Arch.pdf. CUDA Seminar. (Cited on page 20).

[65] E. Rijpkema, K. Goossens, A. Radulescu, J. Dielissen, J. van Meerbergen, P. Wielage, and E. Waterlander. Trade offs in the design of a router with both guaranteed and best-effort services for networks on chip. In Proc. Design, Automation and Test in Europe Conference and Exhibition (DATE), pages 350-355, Washington, D.C., USA, 2003. IEEE Computer Society. ISBN 0-7695-1870-2. doi: 10.1109/DATE.2003.1253633. (Cited on page 16).

[66] J. H. Rutgers. Programming Models for Many-Core Architectures - A Co-design Approach. PhD thesis, University of Twente, May 2014. (Cited on pages 35, 39, 43, 46, and 125).

[67] J. H. Rutgers, M. J. Bekooij, and G. J. Smit. Evaluation of a Connectionless NoC for a Real-Time Distributed Shared Memory Many-Core System. In Proc. Euromicro Symposium on Digital System Design, pages 727-730, Washington, D.C., USA, Sept. 2012. IEEE Computer Society. ISBN 978-0-7695-4798-5. doi: 10.1109/DSD.2012.54. (Cited on pages 8 and 19).

[68] M. J. Rutten, J. T. van Eijndhoven, E. G. Jaspers, P. van der Wolf, E.-J. D. Pol, and O. P. Gangwal. Eclipse : A Heterogeneous Multiprocessor Architecture for Flexible Media. IEEE Design \& Test of Computers, 19(4):39-50, 2002. (Cited on page 27). 
[69] SAC-Research Team. Single assignment c (sac, 2006 (accessed Januari 15, 2015). URL http://www. sac-home.org/. (Cited on page 3).

[70] E. Salminen, A. Kulmala, and T. D. Hamalainen. Survey of Network-on-chip Proposals. White Paper, OCP-IP, pages 1-13, Mar. 2008. (Cited on page 17).

[71] S. L. Shee, A. Erdos, and S. Parameswaran. Architectural Exploration of Heterogeneous Multiprocessor Systems for JPEG. Int'l Journal of Parallel Programming, 36 (1):140-162, Apr. 2007. ISSN 0885-7458. doi: 10.1007/s10766-007-0040-7. (Cited on page 26).

[72] G. J. M. Smit, A. B. J. Kokkeler, P. T. Wolkotte, P. K. F. Hölzenspies, M. D. van de Burgwal, and P. M. Heysters. The Chameleon Architecture for Streaming DSP Applications. EURASIP Journal on Embedded Systems, 2007(1):1-10, Jan. 2007. ISSN 1687-3955. doi: 10.1155/2007/78082. (Cited on pages 17 and 18).

[73] S. Sriram. Embedded Multiprocessors - Scheduling and Synchronization. CRC Press Taylor \& Francis, Boca Raton, FL, USA, 2009. ISBN 978-1-4200-4801-8. (Cited on pages 56 and 81).

[74] R. Stefan, A. Molnos, A. Ambrose, and K. Goossens. A TDM NoC supporting QoS, multicast, and fast connection set-up. In Proc. Design, Automation and Test in Europe Conference and Exhibition (DATE), pages 1283-1288, 2012. ISBN 9783981080186. (Cited on pages 21, 22, and 25).

[75] M. Steine, M. Bekooij, and M. Wiggers. A priority-based budget scheduler with conservative dataflow model. In Proc. Euromicro Symposium on Digital System Design, pages 37-44, New York, NY, USA, 2009. IEEE. doi: 10.1109/DSD.2009.148. (Cited on page 35$)$.

[76] T. Strik and A. Timmer. Heterogeneous multiprocessor for the management of realtime video and graphics streams. Solid-State Circuits, 35(11):1722-1731, 2000. doi: 10.1109/4.881220. (Cited on page 27).

[77] Tilera Corporation. Tile-gx processor family, 2010 (accessed Januari 22, 2015). URL http://www.tilera.com/products/processors/TILE-Gx_Family. (Cited on page 19).

[78] W. Tong, O. Moreira, R. Nas, and K. van Berkel. Hard-Real-Time Scheduling on a Weakly Programmable Multi-core Processor with Application to Multi-standard Channel Decoding. In Proc. IEEE Real-Time and Embedded Technology and Applications Symposium (RTAS), pages 151-160. IEEE, Apr. 2012. ISBN 978-1-4673-0883-0. doi: 10.1109/RTAS.2012.32. (Cited on pages 26 and 27).

[79] J. van den Brand and M. Bekooij. Streaming consistency: a model for efficient MPSoC design. In Proc. Euromicro Symposium on Digital System Design, volume 10, pages 27-31, New York, NY, USA, Aug. 2007. IEEE. ISBN 978-0-7695-2978-3. doi: 10.1109/DSD.2007.4341446. (Cited on page 41).

[80] P. van der Putten and J. Voeten. Specification of Reactive Hardware/Software Systems. $\mathrm{PhD}$ thesis, Technical University of Eindhoven, 1997. (Cited on page 6). 
[81] G. Venkatesh, J. Sampson, N. Goulding, S. Garcia, V. Bryksin, J. Lugo-Martinez, S. Swanson, and M. B. Taylor. Conservation Cores: Reducing the Energy of Mature Computations. In Proc. Int'l Conf. on Architectural Support for Programming Languages and Operating Systems (ASPLOS), pages 205-218, New York, NY, USA, 2010. ACM Press. ISBN 9781605588391. doi: 10.1145/1736020.1736044. (Cited on pages 24 and 26).

[82] M. Westmijze, M. J. G. Bekooij, and G. J. M. Smit. Efficient end-to-end latency distribution analysis for probabilistic time-triggered systems. In Proc. Int'l Conf. on Embedded Computer Systems (SAMOS), pages 290-298, New York, NY, USA, July 2014. IEEE. doi: 10.1109/SAMOS.2014.6893223. (Cited on page 6).

[83] M. H. Wiggers. Aperiodic multiprocessor scheduling for real-time stream processing applications. PhD thesis, University of Twente, June 2009. (Cited on page 6).

[84] M. H. Wiggers, M. J. G. Bekooij, and G. J. M. Smit. Modelling run-time arbitration by latency-rate servers in dataflow graphs. In Proc. Int'l Workshop on Software and Compilers for Embedded Systems (SCOPES), page 11, New York, NY, USA, 2007. ACM Press. doi: 10.1145/1269843.1269846. (Cited on pages 55 and 70).

[85] M. H. Wiggers, M. J. Bekooij, and G. J. Smit. Monotonicity and run-time scheduling. In Proc. Int'l Conf. on Embedded Software (EMSOFT), pages 177-186, New York, NY, USA, 2009. ACM Press. ISBN 9781605586274. doi: 10.1145/1629335.1629359. (Cited on page 80 ).

[86] P. S. Wilmanns, J. P. Hausmans, S. J. Geuns, and M. J. Bekooij. Accuracy improvement of dataflow analysis for cyclic stream processing applications scheduled by static priority preemptive schedulers. In Proc. Euromicro Symposium on Digital System Design, pages 623-630, New York, NY, USA, Aug. 2014. IEEE. doi: 10.1109/DSD.2014.69. (Cited on page 6).

[87] P. Wolkotte, G. Smit, G. Rauwerda, and L. Smit. An Energy-Efficient Reconfigurable Circuit-Switched Network-on-Chip. In Proc. Int'l Parallel and Distributed Processing Symposium (IPDPS), page 155a, New York, NY, USA, 2005. IEEE. ISBN 0-7695-23129. doi: 10.1109/IPDPS.2005.95. (Cited on pages 17, 18, 21, 22, and 25).

[88] Xilinx. LogiCORE IP Fast Simplex Link (FSL) V20 Bus (v2.11c). 2010. (Cited on page 36).

[89] Xilinx. UG761 AXI Reference Guide, volume 761. 2011. (Cited on page 24). 


\section{List of Publications}

[BHJD:1] Jochem H. Rutgers, Stefan J. Geuns, Joost P.H.M. Hausmans, Berend H.J. Dekens, Marco J.G. Bekooij, and Gerard J.M. Smit. Tool for mapping real-time stream processing applications onto a many-core platform. In Proc. Design, Automation and Test in Europe Conference and Exhibition (DATE), March 2012. University Booth.

[BHJD:2] Berend H.J. Dekens, Philip S. Wilmanns, Marco J.G. Bekooij, and Gerard J.M. Smit. Low-cost guaranteed-throughput communication ring for real-time streaming mpsocs. In Proc. Conf. on Design \& Architectures for Signal \& Image Processing (DASIP), pages 239-246, Europe, October 2013. ECSI. ISBN 979-1092279-02-3. URL http://doc . utwente.nl/87860/.

[BHJD:3] Stefan J. Geuns, Berend H.J. Dekens, Philip S. Wilmanns, Joost P.H.M. Hausmans, and Marco J.G. Bekooij. Compiler for mapping stream processing applications onto real-time heterogeneous multiprocessor systems. In Proc. Design, Automation and Test in Europe Conference and Exhibition (DATE), March 2014. University Booth.

[BHJD:4] Berend H.J. Dekens, Philip S. Wilmanns, Marco J.G. Bekooij, and Gerard J.M. Smit. Low-cost guaranteed-throughput dual-ring communication infrastructure for heterogeneous mpsocs. In Proc. Conf. on Design \& Architectures for Signal \& Image Processing (DASIP), Europe, October 2014. ECSI. doi: 10.1109/DASIP.2014.7115628. URL http://doc . utwente.nl/93329/.

[BHJD:5] Guus Kuiper, Berend H.J. Dekens, Stefan J. Geuns, Philip S. Wilmanns, Joost P.H.M. Hausmans, and Marco J.G. Bekooij. Compiler for real-time multiprocessor systems with shared accelerators. In Proc. Design, Automation and Test in Europe Conference and Exhibition (DATE), March 2015. University Booth.

[BHJD:6] Berend H.J. Dekens, Marco J.G. Bekooij, and Gerard J.M. Smit. Low-cost realtime multiprocessor architecture for shared stream processing accelerators. In Reconfigurable Architectures Workshop, RAW 2015, New York, NY, USA, May 2015. IEEE. URL http://doc.utwente.nl/96052/. (accepted). 


\section{THIS THESIS}

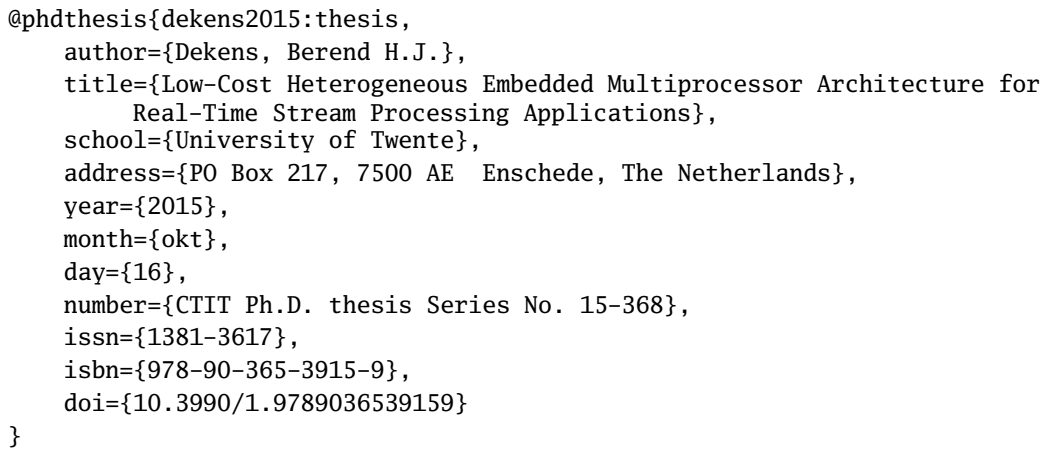

BIBTEX of this thesis 


\section{INDEX}

actors, 54, 67

ALUs, 24

AM, 95

ASIC, 116

B

BE, 17

BER, 94

BIBTEX of this thesis, 150

C-FIFO, 41

C-HEAP, 41

CM, 55

computationally bounded, 93

credit-based flow control, see also creditbased handshake

credit-based handshake, 65

CSDF, vi, $\mathbf{x}, \mathbf{1 8}, 67,81$

\section{D}

directed graph, 54

DMA, 8

DQD, 106

DSP, 15

edges, 54, 67

E

F

FCFS, 44

FEC, 105

FIR, 32

firing duration, 67

firing rule, 54

FM, 103

FSK, 106

FSL, 36
GMSK, 105

GPUs, 20

GT, 17

Guaranteed Acceptance, 40

guaranteed acceptance, 36

$\mathrm{H}$

HSDF, 18

I

ILP, 77

interconnect, see also NoC

ISEs, 26

ISI, 105

K

key-hole, 25, 66

L

LPF, 103

M

master-slave, 9, 23, 43

MCM, 55

MLSE, 105

MPSoCs, 19

MSK, 105

NIs, 10

$\mathrm{NI}, \mathbf{v i}$

NoCs, 8

NoC, $\mathbf{v}$

NUMA, v, ix, 7

OS, 7 
PAL, 64

PCM, 111

152 PEs, v

PE, 10

phases, 67,81

point-to-point, 10, 23, 38

posted writes, 36

predictable, 6

PWM, 111

quanta, 54, 67

$\mathrm{R}$

refinement, 8o, 84, 86

RPCs, 26

$\mathrm{RR}, 37$

RTDMA, 25

S

SDF, vi, $x, 27,54,67$

SDR, v, ix, $\mathbf{3}$

self-edge, 67,81

SIMD, 20

SIMT, 20

SMP, v, ix, 7

SNR, 110

SPMs, 41

TDM, 19

tokens, 54, 67

VA, 105

W

WCETs, 6 
